\title{
Eyes of the needle
}

Citation for published version (APA):

Balthasar, A. J. R. (2018). Eyes of the needle: Spectral tissue sensing, an innovative technology for detecting various tissue types during percutaneous needle-based procedures in locoregional anesthesia and pain medicine. [Doctoral Thesis, Maastricht University]. Datawyse / Universitaire Pers Maastricht. https://doi.org/10.26481/dis.20180323ab

\section{Document status and date:}

Published: 01/01/2018

DOI:

10.26481/dis.20180323ab

\section{Document Version:}

Publisher's PDF, also known as Version of record

\section{Please check the document version of this publication:}

- A submitted manuscript is the version of the article upon submission and before peer-review. There can be important differences between the submitted version and the official published version of record.

People interested in the research are advised to contact the author for the final version of the publication, or visit the DOI to the publisher's website.

- The final author version and the galley proof are versions of the publication after peer review.

- The final published version features the final layout of the paper including the volume, issue and page numbers.

Link to publication

\footnotetext{
General rights rights.

- You may freely distribute the URL identifying the publication in the public portal. please follow below link for the End User Agreement:

www.umlib.nl/taverne-license

Take down policy

If you believe that this document breaches copyright please contact us at:

repository@maastrichtuniversity.nl

providing details and we will investigate your claim.
}

Copyright and moral rights for the publications made accessible in the public portal are retained by the authors and/or other copyright owners and it is a condition of accessing publications that users recognise and abide by the legal requirements associated with these

- Users may download and print one copy of any publication from the public portal for the purpose of private study or research.

- You may not further distribute the material or use it for any profit-making activity or commercial gain

If the publication is distributed under the terms of Article $25 \mathrm{fa}$ of the Dutch Copyright Act, indicated by the "Taverne" license above, 


\section{Eyes of the needle:}

spectral tissue sensing, an innovative technology for detecting various tissue types during percutanous needle-based procedures in locoregional anesthesia and pain medicine

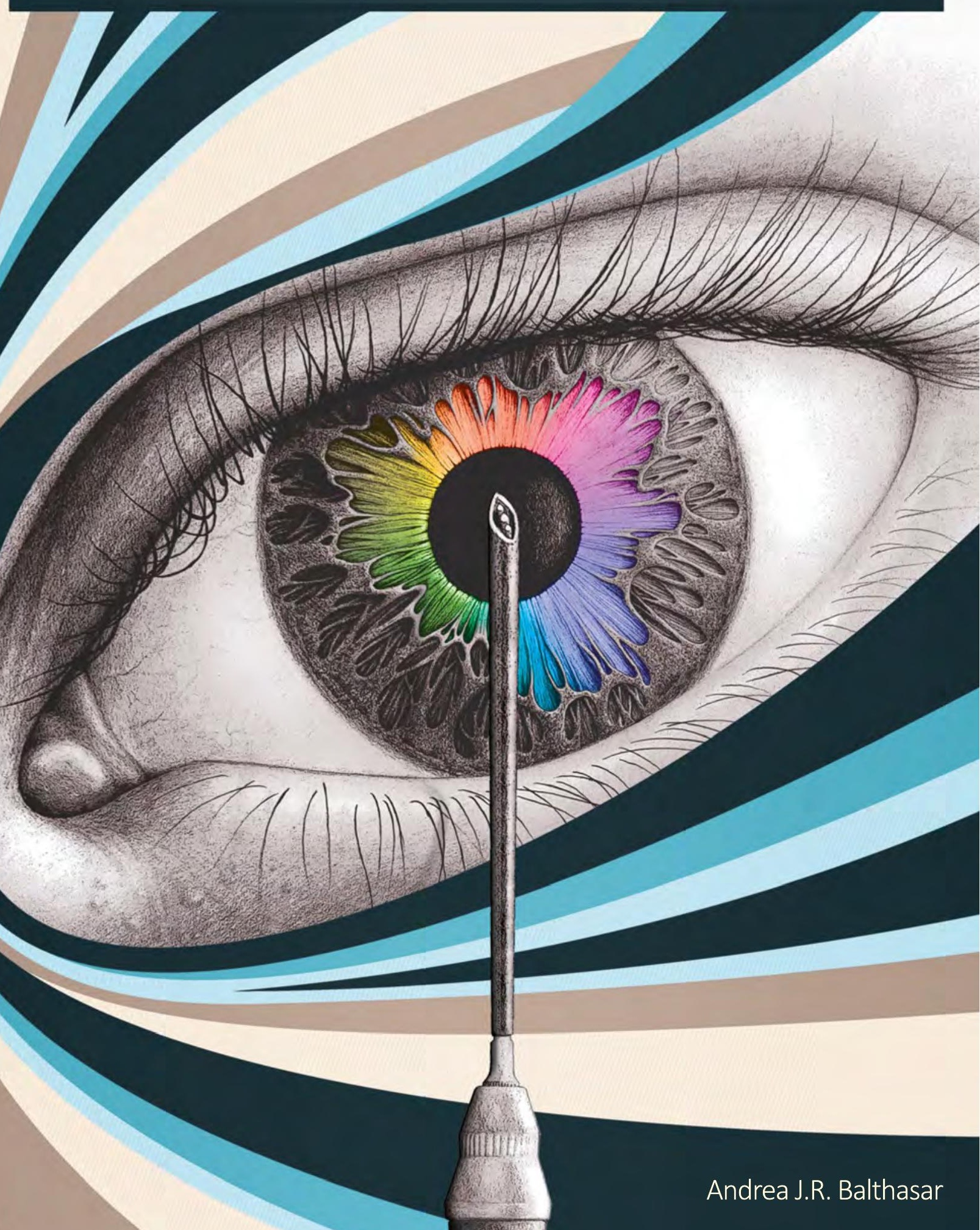




\section{Eyes of the needle}

Spectral tissue sensing, an innovative technology for detecting various tissue types

during percutaneous needle-based procedures in locoregional anesthesia and pain

medicine

\section{PROEFSCHRIFT}

ter verkrijging van de graad van doctor aan de Universiteit Maastricht,

op gezag van de Rector Magnificus,

Prof. dr. Rianne M. Letschert

volgens het besluit van het College van Decanen,

in het openbaar te verdedigen

op vrijdag 23 maart 2018 om 12 uur

door

Andrea J. R. Balthasar,

Geboren op 26 juni 1979, Filderstadt, Duitsland 
Promotor

Prof. dr. Maarten van Kleef

Co-promotor

Dr. Geert-Jan van Geffen (Radboud UMC, Nijmegen)

Beoordelingscommissie

Prof. dr. Nicole Bouvy (voorzitter)

Prof. dr. Walther van Mook

Prof. dr. Yasin Temel

Prof. dr. Kris Vissers (Radboud UMC, Nijmegen)

Prof. dr. André Wolff (UMC Gronigen)

Finacial support for this thesis was provided by Philips Research Eindhoven 
"Wichtig ist, dass man nicht aufhört zu fragen"

Albert Einstein (1879-1955)

German theoretical physicist

To

Ralf and Jarno 



\section{Contents}

Chapter 1 General introduction and aims of the thesis

Chapter 2 What is the best way to analyze optical spectra with the spectral tissue sensing system?

Chapter 3 Is it possible to detect intravascular needle position with the spectral tissue sensing system?

Chapter 3A Is it possible to detect intravascular needle position with the spectral tissue sensing system during nerve blocks?

Chapter 3B Is it possible to identify intra- and extravascular needle placement with the spectral tissue sensing system?

Chapter $4 \quad$ What is the optical signature of nerve tissue?

Chapter 5 Is it possible to detect tissue transitions with the spectral tissue sensing system during peripheral nerve blocks?

Chapter 6 Can the most appropriate and the safest injection environment be determined with the spectral tissue sensing system during lumbar transforaminal epidural injections?

Chapter $7 \quad$ Summary, General Discussion, and Perspectives

Chapter $8 \quad$ Valorization

Chapter $9 \quad$ Acknowledgments

Chapter 10 Curriculum vitae and list of publications 



\section{Chapter \\ General introduction and aims of the thesis}

"A goal without a plan is just a wish"

Antoine de Saint-Exupéry (1900-1944) French writer, poet and aviator 



\section{Introduction}

Identification of the target structure is critical for safe and efficient percutaneous needle-based procedures in regional anesthesia and pain medicine. During several procedures in regional anesthesia and pain medicine, needles are inserted percutaneously, primarily to puncture blood vessels or to target nerves. Catheters are inserted into blood vessels for intravenous medical treatment or measurement of vital parameters, such as blood pressure. ${ }^{1}$ In contrast, nerves are targeted to anesthetize an area for surgery, treat acute postoperative pain, and relieve chronic or neuropathic pain in a certain region. ${ }^{2}$ The development of various imaging techniques and the increasing skill of physicians have rendered diagnostic and therapeutic percutaneous needle-based procedures possible, safer, and more precise. ${ }^{3}$

Over the past 100 years, regional anesthesia and interventional pain treatments have improved with regard to their efficacy and identification of target locations. ${ }^{3}$ Physicians have supported this development by inventing and enhancing imaging techniques and medical devices, such as the nerve stimulator and new types of needles. To understand the need for new technologies and improvements in safety, the history of percutaneous needle procedures and imaging, and the shortcomings and complications of modern techniques must be considered.

The evolution of percutaneous needle-based procedures, imaging techniques, and other medical devices is summarized below. Then, the complications of regional anesthesia and pain interventions, aspects of procedure-related safety, and the sensitivity and specificity of several imaging techniques and medical devices will be discussed. Light as an imaging tool and a new technology, spectral tissue sensing (STS), are introduced and described. Finally, the aim of the thesis and research questions are presented.

\subsection{Safety aspects of regional anesthesia and pain medicine in historical perspective.}

The basis of modern neural blockade is the concept that pain is a sensory warning that is conveyed by specific nerve fibers. Thus, interruption or modulation anywhere in the pathway of a nerve could ease the pain. ${ }^{4}$

One of the first descriptions of locoregional anesthesia dates from 1884. The Austrian ophthalmologist K. Koller (1857-1944) used cocaine as a local anesthetic during eye surgery. ${ }^{5-7}$ The first operation in which 15 mg cocaine was administered intrathecally was performed in 1898 by Bier in a patient with tuberculosis who was scheduled to undergo resection of his left ankle. ${ }^{8,9}$

Clearly, in the early years of locoregional anesthesia, blocks were performed in easily accessible or known anatomical regions. Paresthesia that was reported by the patient 
during the procedure and aspiration before the injections were used for safety reasons and to ensure write needle position.

During history safety improves by means of:

\subsubsection{Nerve stimulator}

In 1912, Georg Clemens Perthes (1869-1927), a German surgeon, first described the use of nerve stimulation. ${ }^{10}$ In 1962, the American anesthesiologists Gordon M. Greenblatt and J.S. Denson reported a system that is similar to the approach that is used today. ${ }^{11}$ The sensitivity of nerve localization increased with the use of a neurostimulator compared with the blind and paresthesia-based techniques. ${ }^{12}$

\subsubsection{Fluoroscopy}

In parallel, fluoroscopy-guided procedures have represented a significant advance in optimizing percutaneous nerve blocks. The first description of using radiography as a diagnostic tool was reported in 1896 by W.C. Roentgen (1845-1923), a German physicist. In 1912, the German surgeon F.F. Härtel (1877-1940) recounted what was likely the first fluoroscopically guided intervention to mitigate pain, detailing a percutaneous approach to the foramen ovale to treat trigeminal neuralgia. ${ }^{13}$

\subsubsection{Computed tomography (CT)}

In 1972, CT was invented by the British engineer G. Hounsfield (1919-2004), who worked for Electric and Musical Industries Ltd. (EMI-Laboratories), and the South African physicist A. McLeod Cormack (1924-1998), of Tufts University. CT allows physician to visualize cross-sections of the body, including bones and organs, and improves percutaneous diagnostic and therapeutic procedures. Curiously enough, there are rumors and controversial reports, which speculate that the development of the CT was probably financed by the profits of the selling of the Beatle's songs by EMI. ${ }^{14}$

\subsubsection{Angiography}

The identification of blood vessels was revolutionized by the advent of contrast fluidbased injections, in combination with radiation. In 1927, the Portuguese physician and neurologist A. Egas Moniz (1874-1955) at the University of Lisbon developed cerebral angiography. The Cuban physician A.W. Castellanos (1902-2000) and 2 American physicians made history in radiology by developing digital subtraction angiography (DSA) in the early 1970s. ${ }^{15}$ The use of contrast fluid during fluoroscopy-based procedures improved periprocedural blood vessel identification significantly. 


\subsubsection{Ultrasonography}

The most recent advance in imaging and anesthetic procedures has been the use of ultrasonography. In 1978, South African anesthesiologist P. La Grange and colleagues were the first to perform ultrasonography for peripheral nerve blockade, using a Doppler ultrasonography transducer to localize the subclavian artery to facilitate the introduction of supraclavicular brachial plexus blocks. ${ }^{16}$ In 1989, P. Ting, a Singaporean anesthesiologist, reported the use of B-mode ultrasonography to demonstrate the anatomy of the axilla and monitor the spread of local anesthetics. ${ }^{17}$ With ultrasonography, nerves can be visualized without ionizing radiation or MRI. Mobile ultrasonography machines are used widely in the operating theater, rendering locoregional blocks safer and more efficient. ${ }^{18,19}$ Due to the introduction of ultrasonography in regional anesthesia, new techniques and approaches are described, and old techniques are revived. ${ }^{20,21}$

\subsubsection{Needles and syringes}

Paralleling the development of imaging devices, various needle types and syringes were invented to reduce complications, such as post dural puncture headache, and ease the visualization of the needle during ultrasonography-guided procedures. ${ }^{22,23}$

\subsubsection{Magnetic resonance imaging}

Yet, magnetic resonance imaging (MRI) and functional MRI have had less of an influence on the growth of needle-guided procedures in regional anesthesia and pain medicine. Figure 1.1 gives an overview of the times at which imaging tools were implemented in anesthesia practices.

In summary, over the past 100 years, nerve stimulation, fluoroscopy, ultrasonography, $\mathrm{CT}$, and DSA have gained a place in percutaneous needle-based procedures, improving safety and contributing to the creation of less invasive techniques and novel treatments. 


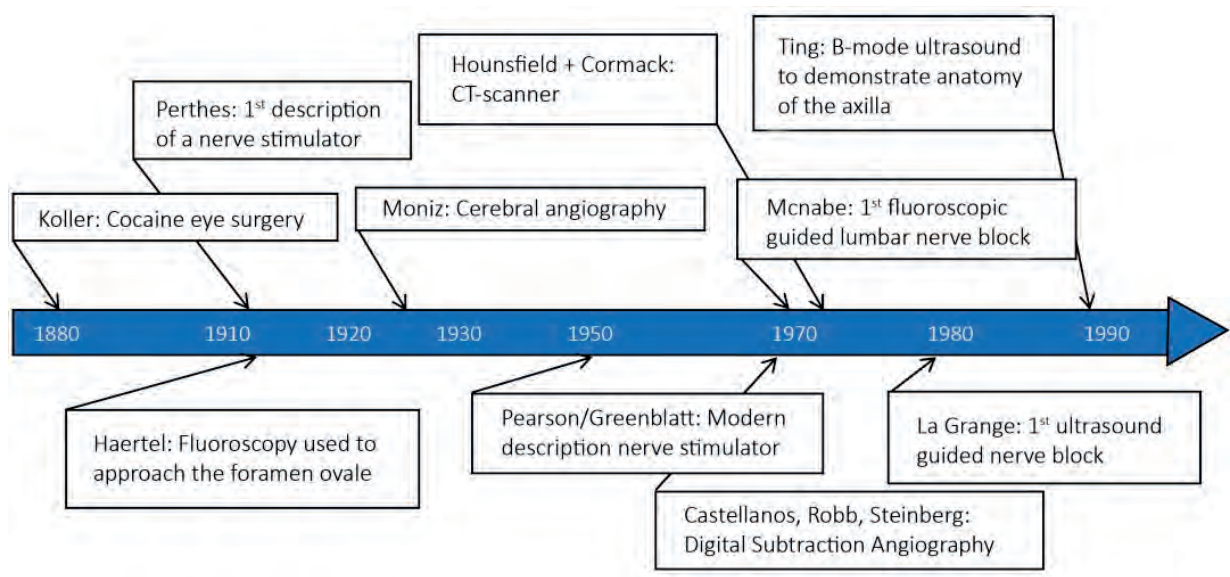

Anatomical and pharmacological knowledge, needle development, aspiration

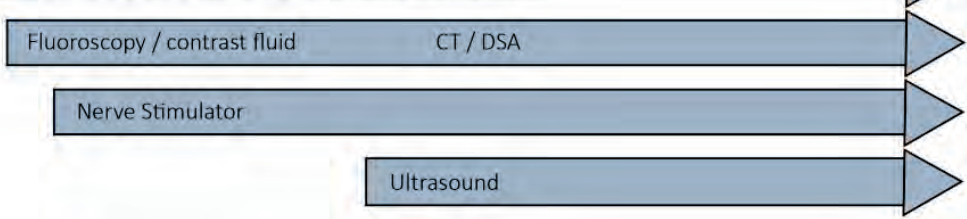

Figure 1.1 Historical development of tools and imaging techniques for percutaneous needle-based procedures.

\subsection{Complications of regional anesthesia and pain medicine}

Clear identification of the target structure and a detailed procedure are of paramount importance with regard to the performance of percutaneous needle insertions. These factors increase the success of the therapy, improve the quality of nerve blocks, and decrease the incidence of complications. Current localization techniques and medical devices help the clinician guide the needle to the target. Nevertheless, serious complications during regional anesthesia and interventional pain treatments still occur. ${ }^{24-26}$

Neural structures can be damaged by direct needle trauma or indirectly by ischemia due to vascular insufficiency or injection of agents at the wrong location. In $15 \%$ of patients who undergo peripheral nerve block, mild paresthesia occur during or after the procedure. Most of these symptoms resolve within days to weeks. Serious neurological injuries develop in 2.4 per 10,000 peripheral nerve blocks. ${ }^{27,28}$ The brachial plexus is the most commonly injured peripheral nerve structure, based on a closed claims analysis. ${ }^{29}$

During interventional pain therapy, more serious complications have been reported for procedures at the spinal level. Transforaminal epidural injections with local anesthetics and certain corticosteroids have been administered as standard therapy for radicular pain. In the past several decades, the incidence of severe complications of interventional pain therapy has risen-spinal infarctions at the lumbar level and cerebral 
infarction and death after cervical injections have been reported. ${ }^{30}$ One of the mechanisms could be unnoticed arterial uptake of certain corticosteroids, effecting arterial occlusion. $^{31-33}$

\subsection{Safety aspects of interventional procedures}

To avoid serious adverse events, several precautions should be taken during the procedure. An overview of the key safety issues in interventional pain medicine and regional anesthesia is presented here.

\subsubsection{Patient-related factors}

The patient history should be checked among other things for anticoagulant use, diabetes mellitus, allergies, and anatomical variations. Informed consent should be obtained, and the correct side of the procedures should be clearly marked. ${ }^{34}$ Although this issue is debated, it is preferred that the patient should be awake during the procedure to provide feedback and report symptoms to the treating physician, such as pain during injection, metallic taste, blurred vision, difficulty speaking, and dizziness, indicating that neurologic complications may occur. ${ }^{18,26}$

\subsubsection{Skills of the physician}

The physician should be well trained in the technique and understand the medical equipment and relevant anatomical structures in the procedure. ${ }^{35}$ The use of phantoms and practical simulations can improve needle handling during ultrasonography-guided needle use. ${ }^{36,37}$ Certification of physicians could be a valuable tool to keep their knowledge current and ensure safety. ${ }^{35,38,39}$

\subsubsection{Location, monitoring, and equipment}

Standard vital signs of the patient should be monitored (ECG, blood pressure, oxygen saturation), intravenous access should be obtained, and the equipment for performing resuscitative maneuvers should be in close proximity. ${ }^{26,29,40}$ The equipment and medical devices should be appropriate, checked, and up to date. Intralipid should be available in case local anesthetic systemic toxicity occurs. ${ }^{41}$

\subsubsection{Trajectory of the needle}

The trajectory of the needle should be safe, and unnecessary punctures of vital structures, such as blood vessels and organs, should be avoided. Anatomical landmarks, fluoroscopy, injection of contrast fluid, nerve stimulation, and ultrasonographic images should be used to identify the target structure. ${ }^{35,42}$ 


\subsubsection{At the target}

Before local anesthetics are injected or radiofrequency treatments are performed, several precautions must be taken. First, aspiration, live fluoroscopy with contrast fluid injection, or DSA should be performed to exclude unintended intravascular needle positions as much as possible. ${ }^{43}$ Next, in certain cases, nerve stimulation is advised to ensure the correct needle position; this step provides functional information. ${ }^{44}$ Finally, only a small dose of fluid is injected, while the spreading is observed, before more local anesthetic is given. ${ }^{45}$

These techniques and medical devices might be insufficient to prevent all adverse events, possibly due to the inadequate sensitivity and specificity of the approaches.

\subsection{Sensitivity and specificity of localization techniques}

\subsubsection{Blood vessel identification:}

Aspiration identifies the intravascular needle position in $25 \%$ to $47 \%$ of cases. ${ }^{46}$

Injection of contrast fluid during live fluoroscopy detects the intravascular needle position in $60 \%$ to $71 \%$ of cases compared with digital subtraction angiography (DSA). ${ }^{46-48}$

Currently, DSA is recommended to exclude intravascular needle placements during pain interventions. ${ }^{48,49}$ Despite the use of DSA, case reports of intravascular injection have been reported. ${ }^{50}$

Ultrasonography can identify blood vessels and discriminate between artery and veins. Also, needle tips and catheters can be visualized during the procedure in blood vessels. Further, combined with aspiration and the injection of small amounts of fluid (hydrodissection), the correct needle tip placement can be verified. ${ }^{51}$ Despite the use of ultrasonography, the injection of local anesthetics into blood vessels can still occur. ${ }^{52}$

\subsubsection{Nerve identification}

Paresthesia has been found to be $38.2 \%$ sensitive in determining needle-nerve proximity. ${ }^{12}$

Nerve stimulation in locoregional anesthesia identifies the nerve in $74.5 \%$ of cases. ${ }^{12}$

Ultrasonography can identify nerves and is used widely during locoregional anesthesia. ${ }^{35}$ The identification of nerves with ultrasonography depends highly on the anatomy, the echogenic characteristics of the patient and nerves, and the interpretation of the ultrasound images by the physician. ${ }^{53-55}$ Ultrasonography guidance has increased the safety of regional anesthesia. ${ }^{54,56,57}$ The sensitivity and specificity of ultrasonography in nerve detection have not been reported, nor have the sonographic characteristics of a safe injection. ${ }^{35,58}$ Several studies show that unintended subepineural injection still occurs. $^{59-61}$ 
Fluoroscopy fails to identify nerves directly. Only after contrast fluid injection can the correct needle position be confirmed through spreading of the fluid along the nerve. $^{44}$

\subsubsection{Human factor}

Physician- Another reason for complications or procedure failure is the human factor. Ultrasonography, DSA, and fluoroscopic images are highly dependent on the interpretation and experience of the physician. ${ }^{39}$ Ultrasonography with or without nerve stimulation is currently the most appropriate technique during peripheral nerve blocks. ${ }^{62,63}$

\subsubsection{Summary}

Most of the complications of regional anesthesia and pain medicine are attributed to the limitations of current imaging techniques and medical devices and the misinterpretation of information. There is no ideal medical device. New techniques are necessary to address the shortcomings of existing techniques.

\subsection{Light as an imaging tool}

In biomedical optics, many disciplines are cooperating to develop innovative technologies that use light. This interdisciplinary field includes optical engineering, biophysics, computer science, medicine, biology, and chemistry. ${ }^{64}$ Fluorescence, spectroscopy, and Raman (vibrational spectroscopy) are some of the more popular optical techniques that are used in a clinical setting. ${ }^{64}$

This thesis will examine diffuse reflectance spectroscopy (DRS) as an imaging tool. DRS is the basis of a system, called spectral tissue sensing (STS). To understand the principles of DRS, the physical definition of light and spectroscopy must first be introduced.

\subsubsection{Light and spectroscopy}

Light is defined as an electromagnetic wave that has the properties of particles and is produced by atoms and accelerating charges. Electromagnetic waves are described as sinusoidal waves, allowing standard wave parameters, such as wavelength and frequency, to be characterized. Wavelength is the spatial period of the wave- i.e., the distance over which the wave's shape repeats. Frequency is defined as the number of oscillations per second. The electromagnetic spectrum is the range of all possible frequencies of electromagnetic radiation. The electromagnetic spectrum extends from below the low frequencies that are used for modern communication to gamma radiation at the shortwavelength (high-frequency) end, encompassing wavelengths from thousands of kilometers down to a fraction of the size of an atom. ${ }^{65,66}$

Most segments of the electromagnetic spectrum in science are used for spectroscopic and other probing interactions to analyze matter. Spectroscopy is the study of 
the interaction between matter and electromagnetic radiation. ${ }^{65}$ Electromagnetic waves are used widely in medicine for diagnostic and monitoring purposes- one such well-known method is pulse oximetry. ${ }^{67,68}$ Figure 1.2 gives an overview of the electromagnetic spectrum and indicates the wavelengths that are discussed in this thesis.

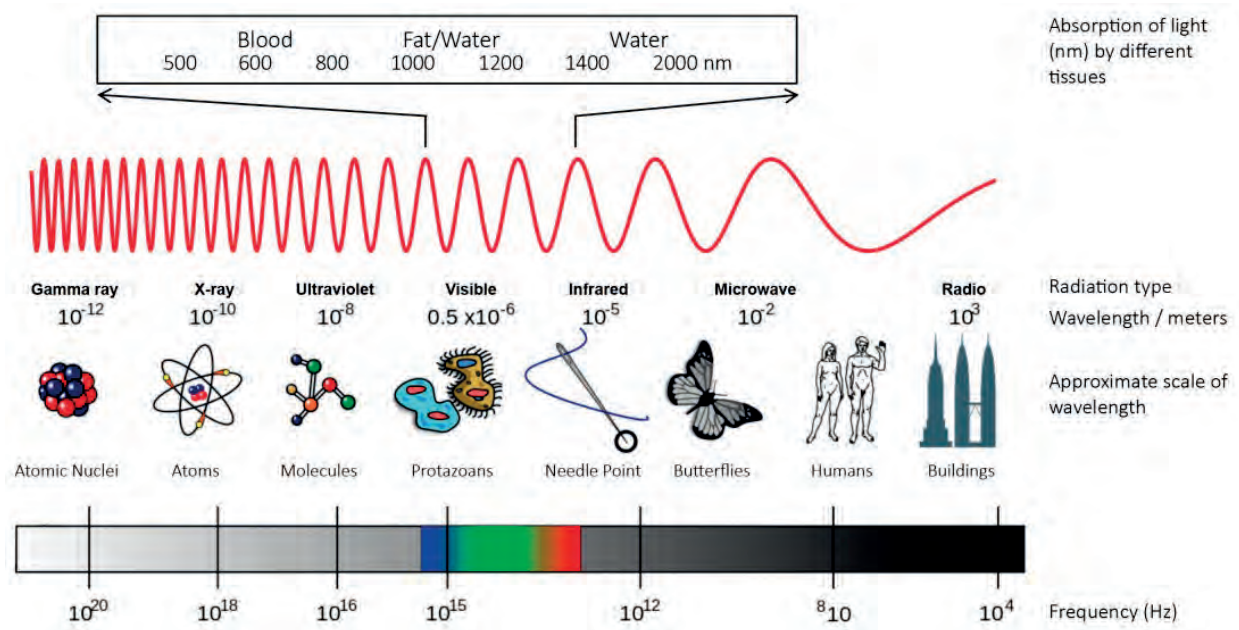

Figure 1.2 Overview of the electromagnetic spectrum. The range of wavelengths (500 nm to $2000 \mathrm{~nm}$ ) of the light that is used in this thesis spans the visible and infrared spectra and is indicated at the top of the figure (source: wikimedia.org ${ }^{69}$ ).

\subsubsection{Spectral tissue sensing}

The principle of spectral tissue sensing (STS) is as follows: when tissue is illuminated, part of the light is absorbed, and some of it is reflected. The light that is used for the studies in this thesis has a range of wavelengths from 500 to 2000 nanometers. Figure 1.2 gives an overview of the electromagnetic spectrum.

This thesis examines a STS system that is based on diffuse reflectance spectroscopy (DRS). ${ }^{70-73}$ If the spectrum of the light that is reflected from the tissue is analyzed, the characteristics of the tissue can be obtained. Thus, the spectrum that is measured can be used to analyze the composition of the tissue. Also, as detailed further in this thesis, if databases of spectra of many tissues are built, methods can be developed to recognize various tissues, based on their STS signatures.

Figure 1.3 shows a schematic of a STS system. This system comprises a console and a needle. The needle that is connected to the STS console contains 2 integrated optical fibers - one for illuminating the tissue and the other to collect reflected light from the tissue. The console of the STS system harbors a light source that delivers light in the 500-1600 $\mathrm{nm}$ wavelength range to the tissue via the illuminating fiber in the needle. The reflected light that is measured is then converted into levels of physiological parameters, such as hemoglobin (oxygenated and deoxygenated), water, and lipids, and 
into the amount of scatter. Scattering is related to refractive index changes present in the tissue i.e. to the inhomogeneity of the tissue. Also, the measured spectrum can be compared against a database of tissue spectra that are stored in the system to determine tissue type. The results of the analyses that are performed by the STS system are displayed on a screen that is viewed by the physician.

Depending on the environment in which the system is used, the spectral databases that are stored in the system, the analyses, and the information that is displayed on the screen can be adapted to the needs of the user. The volume of tissue which is measured by the needles that are used in this thesis is approximately 1 cubic millimeter.

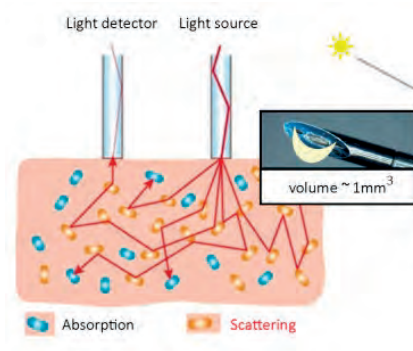

A

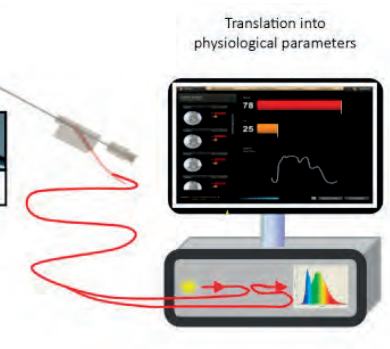

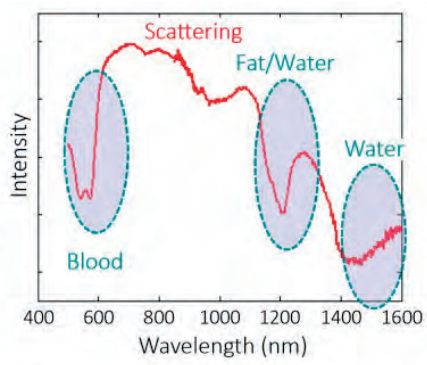

D

Figure 1.3 Overview of the spectral tissue sensing system.

A: Two fibers (an illuminating fiber and a light-collecting fiber) are integrated in a needle; the basic principles of diffuse reflectance spectroscopy (DRS) (absorption and scattering) are shown. B: A photograph of an actual needle tip, the 2 fibers, and the injection lumen. The sample volume is roughly $1 \mathrm{~mm}^{3}$.

C: The console contains a light source to illuminate the tissue via the first fiber and a detector for capturing the reflected light from the tissue via the second fiber. In this case, the spectrum and physiological parameters that are determined are displayed on the screen.

$D$ : A reflectance spectrum: a graph of the measured intensity of reflected light on the $y$-axis versus the wavelength, in nanometers $(\mathrm{nm})$, on the $\mathrm{x}$-axis. Typical absorptions of light for blood, water, and fat are indicated by the blue ovals. The shape of the spectrum also indicates the amount of scatter.

\subsubsection{STS and percutaneous needle-based procedures}

STS might have value in percutaneous needle-based interventional medical procedures and their safety. Current techniques can't identify various tissues directly. STS that is integrated into a needle can extend the operator's view and generate independent information on the type of tissue in front of the needle tip. STS exploits the phenomenon that biological tissues are optically inhomogeneous. Light propagation within a tissue depends on the scattering and absorption properties of its components, such as cells, cell organelles, and various fiber structures. ${ }^{74}$ Several questions must be answered during the development of a new clinically important and useful imaging tool. 
First, the ideal method of analyzing optical data that are generated by STS should be defined. The significant diversity and structural complexity of tissues impede the development of adequate optical models for tissue recognition. A database of the optical characteristics of clinically relevant tissues should be established.

Second, the tissue types that should be detected must be defined to reduce complications. To be a valuable additive device, STS should identify the intravascular needle position clearly. As discussed, there remain serious complications of intravascular injections of medication. ${ }^{28,61,75}$ Additionally, the reliable identification of nerve tissue can improve the safety and efficacy of procedures in locoregional anesthesia and pain medicine. Experts are debating the ideal needle tip position for anesthetizing nerves. ${ }^{58,59}$ However, it is important to avoid any nerve injury by the needle during the procedure. STS could support physicians if it can identify fascicular nerve tissue.

Third, during needle-based procedures, the needle trajectory is paramount. STS can support the operator by monitoring the transition of the needle between tissues during the puncture. Anatomical interpretation of the image and needle tip identification are some of the more challenging aspects of ultrasonography-based procedures. The transition from superficial subcutaneous fat to muscle tissue and nerve tissue should be recognized. Thus, the optical characteristics of such tissues must be defined. STS can support the interpretation of the information that is generated by the ultrasonography image by alerting one to transition of the needle tip through tissues. Operators could be reassured by the STS findings when they advance the needle to the target.

Altogether, a safe target area for injection during needle-based percutaneous procedures must be identified. 


\subsection{Aim of the thesis and research questions}

The aim of this thesis is to evaluate spectral tissue sensing in percutaneous needlebased procedures. The following research questions were raised:

1. What is the best way to analyze optical spectra with the STS system?

2. Is it possible to detect intravascular needle position with the STS system?

3. What is the optical signature of nerve tissue?

4. Is it possible to detect tissue transitions with the STS system during peripheral nerve blocks?

5. Can the most appropriate and the safest injection environment be determined with the STS system during lumbar transforaminal epidural injections?

The first research question will be answered in an ex vivo human study. Different algorithms were tested. With the obtained data, the sensitivity and specificity of the STS can be calculated, wherein the STS may differentiate nerves from the surrounding tissue (Chapter 2).

The second research question will be answered in two different settings. One study is in patients, and the other study is performed in volunteers. Moreover, in other studies of this thesis, blood vessel detection was measured as a secondary outcome (Chapter 3).

Due to ethical reasons, intended nerve puncture is not possible in an in vivo setting. Therefore, in order to answer the third question, we will obtain nerve tissue from ex vivo human nerves, and the optical tissue characteristics will be examined (Chapter 4).

In clinical settings, the last two questions will be answered. During ultrasoundguided peripheral nerve blocks, different spectra were analyzed with the needle tip in different tissues (Chapter 5).

The last question will be answered in a chronic pain patient population. During a standard procedure, STS was compared with fluoroscopy and DSA (Chapter 6). 


\section{References}

1. Kim SH, Lilot M, Sidhu KS, Rinehart J, Yu Z, Canales C, et al. Accuracy and precision of continuous noninvasive arterial pressure monitoring compared with invasive arterial pressure: a systematic review and meta-analysis. Anesthesiology. 2014;120(5):1080-97.

2. Ilfeld BM, Morey TE, Wright TW, Chidgey LK, Enneking FK. Continuous interscalene brachial plexus block for postoperative pain control at home: a randomized, double-blinded, placebo-controlled study. Anesthesia and analgesia. 2003;96(4):1089-95, table of contents.

3. Walker KJ, McGrattan K, Aas-Eng K, Smith AF. Ultrasound guidance for peripheral nerve blockade. Cochrane Database Syst Rev. 2009(4):CD006459.

4. Manchikanti L, Boswell MV, Raj PP, Racz GB. Evolution of interventional pain management. Pain physician. 2003;6(4):485-94.

5. Liljestrand G. Carl Koller and the development of local anesthesia. Acta physiologica Scandinavica Supplementum. 1967;299:1-30.

6. Goerig M, Bacon D, van Zundert A. Carl Koller, cocaine, and local anesthesia: some less known and forgotten facts. Regional anesthesia and pain medicine. 2012;37(3):318-24.

7. Koller K. Ueber die Verwendung des Kokains zur Anaesthesierung am Auge. Wiener Medizinische Wochenschrift. 1884;34:1276-309.

8. Bier A. Ueber einen neuen Weg Lokalanesthesie in den gliedmassen zu erzeugen. Langenbeck's Archiv fuer klinische Chirurgie. 1908;86:1007-16.

9. Wulf HF. The centennial of spinal anesthesia. Anesthesiology. 1998;89(2):500-6.

10. Perhtes. Leitungsanesthesie unter zuhilfename elektrischer Reizung. Muenchen Medizinische Wochenschrift. 1912;47:2545-8.

11. Greenblatt GM, Denson JS. Needle nerve stimulatorlocator: nerve blocks with a new instrument for locating nerves. Anesthesia and analgesia. 1962;41:599-602.

12. Perlas A, Niazi A, McCartney C, Chan V, Xu D, Abbas S. The sensitivity of motor response to nerve stimulation and paresthesia for nerve localization as evaluated by ultrasound. Regional anesthesia and pain medicine. 2006;31(5):445-50.

13. Hartel F. Die Leitungsanesthesie und Injectionsbehandlung des Ganglion Gasserie und der Trigeminusaeste. Arch Klin Chir. 1912;100:193-292.

14. Goodman LR. The Beatles, the Nobel Prize, and CT scanning of the chest. Radiol Clin North Am 2010; 48:1-7.

15. Mistretta CA, Grist TM. X-ray digital subtraction angiography to magnetic resonance-digital subtraction angiography using three-dimensional TRICKS. Historical perspective and computer simulations: a review. Investigative radiology. 1998;33(9):496-505.

16. la Grange P, Foster PA, Pretorius LK. Application of the Doppler ultrasound bloodflow detector in supraclavicular brachial plexus block. British journal of anaesthesia. 1978;50(9):965-7.

17. Ting PL, Sivagnanaratnam V. Ultrasonographic study of the spread of local anaesthetic during axillary brachial plexus block. British journal of anaesthesia. 1989;63(3):326-9.

18. Neal JM, Brull R, Horn JL, Liu SS, McCartney CJ, Perlas A, et al. The Second American Society of Regional Anesthesia and Pain Medicine Evidence-Based Medicine Assessment of Ultrasound-Guided Regional Anesthesia: Executive Summary. Regional anesthesia and pain medicine. 2016;41(2):181-94.

19. Lewis SR, Price A, Walker KJ, McGrattan K, Smith AF. Ultrasound guidance for upper and lower limb blocks. Cochrane Database Syst Rev. 2015(9):CD006459.

20. Marhofer P, Harrop-Griffiths W, Kettner SC, Kirchmair L. Fifteen years of ultrasound guidance in regional anaesthesia: part 1. Br J Anaesth. 2010;104(5):538-46.

21. Marhofer P, Harrop-Griffiths W, Willschke H, Kirchmair L. Fifteen years of ultrasound guidance in regional anaesthesia: Part 2-recent developments in block techniques. Br J Anaesth. 2010;104(6):673-83. 
22. Uppal V, Sondekoppam RV, Ganapathy S. Effect of beam steering on the visibility of echogenic and nonechogenic needles: a laboratory study. Canadian journal of anaesthesia = Journal canadien d'anesthesie. 2014;61(10):909-15.

23. Ip VH, Tsui BC. Practical concepts in the monitoring of injection pressures during peripheral nerve blocks. International anesthesiology clinics. 2011;49(4):67-80.

24. Neal JM, Brull R, Chan VW, Grant SA, Horn JL, Liu SS, et al. The ASRA evidence-based medicine assessment of ultrasound-guided regional anesthesia and pain medicine: Executive summary. Regional anesthesia and pain medicine. 2010;35(2 Suppl):S1-9.

25. Sites BD, Brull R, Chan VW, Spence BC, Gallagher J, Beach ML, et al. Artifacts and pitfall errors associated with ultrasound-guided regional anesthesia: Part II: A pictorial approach to understanding and avoidance. Regional anesthesia and pain medicine. 2010;35(2 Suppl):S81-92.

26. Neal JM, Bernards CM, Hadzic A, Hebl JR, Hogan QH, Horlocker TT, et al. ASRA Practice Advisory on Neurologic Complications in Regional Anesthesia and Pain Medicine. Regional anesthesia and pain medicine. 2008;33(5):404-15.

27. Liguori GA. Complications of regional anesthesia: nerve injury and peripheral neural blockade. Journal of neurosurgical anesthesiology. 2004;16(1):84-6.

28. Auroy Y, Benhamou D, Bargues L, Ecoffey C, Falissard B, Mercier FJ, et al. Major complications of regional anesthesia in France: The SOS Regional Anesthesia Hotline Service. Anesthesiology. 2002;97(5):1274-80.

29. Lee LA, Posner KL, Kent CD, Domino KB. Complications associated with peripheral nerve blocks: lessons from the ASA Closed Claims Project. International anesthesiology clinics. 2011;49(3):56-67.

30. Rathmell JP. Toward improving the safety of transforaminal injection. Anesthesia and analgesia. 2009;109(1):8-10.

31. Houten JK, Errico TJ. Paraplegia after lumbosacral nerve root block: report of three cases. Spine J. 2002;2(1):70-5.

32. Helm S, Glaser S, Falco F, Henry B. A medical-legal review regarding the standard of care for epidural injections, with particular reference to a closed case. Pain physician. 2010;13(2):145-50.

33. Huntoon MA, Martin DP. Paralysis after transforaminal epidural injection and previous spinal surgery. Regional anesthesia and pain medicine. 2004;29(5):494-5.

34. Slocombe P, Pattullo S. A site check prior to regional anaesthesia to prevent wrong-sided blocks. Anaesth Intensive Care. 2016;44(4):513-6.

35. Abdallah FW, Macfarlane AJ, Brull R. The Requisites of Needle-to-Nerve Proximity for Ultrasound-Guided Regional Anesthesia: A Scoping Review of the Evidence. Regional anesthesia and pain medicine. 2016;41(2):221-8.

36. Sites BD, Gallagher JD, Cravero J, Lundberg J, Blike G. The learning curve associated with a simulated ultrasound-guided interventional task by inexperienced anesthesia residents. Regional anesthesia and pain medicine. 2004;29(6):544-8.

37. Barrington MJ, Wong DM, Slater B, Ivanusic JJ, Ovens M. Ultrasound-guided regional anesthesia: how much practice do novices require before achieving competency in ultrasound needle visualization using a cadaver model. Regional anesthesia and pain medicine. 2012;37(3):334-9.

38. Sujatta S. First of all: Do not harm! Use of simulation for the training of regional anaesthesia techniques: Which skills can be trained without the patient as substitute for a mannequin. Best practice \& research Clinical anaesthesiology. 2015;29(1):69-80.

39. Dickerson J, Paul K, Vila P, Whiticar R. The role for peer-assisted ultrasound teaching in medical school. The clinical teacher. 2016.

40. Fitzgibbon DR, Posner KL, Domino KB, Caplan RA, Lee LA, Cheney FW. Chronic pain management: American Society of Anesthesiologists Closed Claims Project. Anesthesiology. 2004;100(1):98-105.

41. Cave G, Harvey M. Intravenous Lipid Emulsion as Antidote Beyond Local Anesthetic Toxicity: A Systematic Review. Acad Emerg Med. 200916(9):815-24.

42. Gadsden J, Latmore M, Levine DM. Evaluation of the eZono 4000 with eZGuide for ultrasound-guided procedures. Expert review of medical devices. 2015;12(3):251-61. 
43. J. van Zundert JP, C.T. Hartrick, A. Lataster, F.J.P.M. Huygen, N. Mekhail, M. van Kleef. Evidence Based Interventional Pain Medicine - According to Clinical Diagnoses. First ed. Oxford: Wiley-Blackwell; 2012.

44. Van Boxem K, van Bilsen J, de Meij N, Herrler A, Kessels F, Van Zundert J, et al. Pulsed radiofrequency treatment adjacent to the lumbar dorsal root ganglion for the management of lumbosacral radicular syndrome: a clinical audit. Pain Med. 2011;12(9):1322-30.

45. Dufour E, Donat N, Jaziri S, Kurdi O, Couturier C, Dreyfus JF, et al. Ultrasound-guided perineural circumferential median nerve block with and without prior dextrose $5 \%$ hydrodissection: a prospective randomized double-blinded noninferiority trial. Anesthesia and analgesia. 2012;115(3):728-33.

46. Furman MB, Giovanniello MT, O'Brien EM. Incidence of intravascular penetration in transforaminal cervical epidural steroid injections. Spine. 2003;28(1):21-5.

47. Lee MH, Yang KS, Kim YH, Jung HD, Lim SJ, Moon DE. Accuracy of live fluoroscopy to detect intravascular injection during lumbar transforaminal epidural injections. The Korean journal of pain. 2010;23(1):18-23.

48. Hong JH, Huh B, Shin HH. Comparison Between Digital Subtraction Angiography and Real-time Fluoroscopy to Detect Intravascular Injection During Lumbar Transforaminal Epidural Injections. Regional anesthesia and pain medicine. 2014.

49. Jasper JF. Role of digital subtraction fluoroscopic imaging in detecting intravascular injections. Pain physician. 2003;6(3):369-72.

50. Chien GC, Candido KD. Digital Subtraction Angiography is Not the Answer for Safe Epidural Injections. Pain physician. 2014;17(3):E413-4.

51. Auyong DB, Benonis JG, Gonzales J. Distal squeeze technique increases venous blood flow and allows for easy identification of veins with ultrasound. Regional anesthesia and pain medicine. 2010;35(3):314.

52. Sites BD, Taenzer AH, Herrick MD, Gilloon C, Antonakakis J, Richins J, et al. Incidence of local anesthetic systemic toxicity and postoperative neurologic symptoms associated with 12,668 ultrasound-guided nerve blocks: an analysis from a prospective clinical registry. Regional anesthesia and pain medicine. 2012;37(5):478-82.

53. O'Neil JMD. Musculoskeletal Ultrasound, Anatomy and Technique. 1 ed. New York: Spinger-Verlag New York; 2008. 348 p.

54. Neal JM. Ultrasound-guided regional anesthesia and patient safety: An evidence-based analysis. Regional anesthesia and pain medicine. 2010;35(2 Suppl):S59-67.

55. Orebaugh SL, Kentor ML, Williams BA. Adverse outcomes associated with nerve stimulator-guided and ultrasound-guided peripheral nerve blocks by supervised trainees: update of a single-site database. Regional anesthesia and pain medicine. 2012;37(6):577-82.

56. Barrington MJ, Kluger R. Ultrasound guidance reduces the risk of local anesthetic systemic toxicity following peripheral nerve blockade. Regional anesthesia and pain medicine. 2013;38(4):289-99.

57. Hadzic A, Sala-Blanch X, Xu D. Ultrasound guidance may reduce but not eliminate complications of peripheral nerve blocks. Anesthesiology. 2008;108(4):557-8.

58. Bigeleisen PE. Nerve puncture and apparent intraneural injection during ultrasound-guided axillary block does not invariably result in neurologic injury. Anesthesiology. 2006;105(4):779-83.

59. Orebaugh SL, McFadden K, Skorupan H, Bigeleisen PE. Subepineurial injection in ultrasound-guided interscalene needle tip placement. Regional anesthesia and pain medicine. 2010;35(5):450-4.

60. Chin KJ, Perlas A, Chan VW, Brull R. Needle visualization in ultrasound-guided regional anesthesia: challenges and solutions. Regional anesthesia and pain medicine. 2008;33(6):532-44.

61. Liu SS, YaDeau JT, Shaw PM, Wilfred S, Shetty T, Gordon M. Incidence of unintentional intraneural injection and postoperative neurological complications with ultrasound-guided interscalene and supraclavicular nerve blocks. Anaesthesia. 2011;66(3):168-74.

62. Terkawi AS, Karakitsos D, Elbarbary M, Blaivas M, Durieux ME. Ultrasound for the anesthesiologists: present and future. ScientificWorldJournal. 2013;2013:683685.

63. Neuburger M, Schwemmer U, Volk T, Gogarten W, Kessler P, Steinfeldt T. [Localization of peripheral nerves. Success and safety with electrical nerve stimulation]. Anaesthesist. 2014;63(5):422-8.

64. Valdes PA, Roberts DW, Lu FK, Golby A. Optical technologies for intraoperative neurosurgical guidance. Neurosurg Focus. 2016;40(3):E8. 
65. Douglas A. Skoog DMW, F. James Holler, Stanley R. Coruch. Fundamentals of Analytical Chemistry. 8th ed. Belmont, CA 94002-3098, USA: MaryFinch; 2014. 1048 p.

66. Sliney DH. What is light? The visible spectrum and beyond. Eye (Lond). 2016;30(2):222-9.

67. Levin PD, Levin D, Avidan A. Medical aerosol propellant interference with infrared anaesthetic gas monitors. Br J Anaesth. 2004;92(6):865-9.

68. Chan ED, Chan MM, Chan MM. Pulse oximetry: understanding its basic principles facilitates appreciation of its limitations. Respir Med. 2013;107(6):789-99.

69. wikimedia.

https://upload.wikimedia.org/wikipedia/commons/thumb/c/cf/EM_Spectrum_Properties_edit.svg/2000 px-EM_Spectrum_Properties_edit.svg.png [website].

70. Schenkman KA, Marble, DR., et al. Near infrared spectroscopic measurement of myoglobin oxygen saturation in the presence of hemboglobin using partial least-squares analysis. Applied Spectroscopy. 1999;53(3):325-31.

71. Bashkatov AN, Genina E.A., et al. Optical properties of the subcutaneous adipose tissue in the spectral range 400-2500 $\mathrm{nm}$. Optics and Spectroscopy. 2005;99(5):836-42.

72. Bashkatov AN, Genina E.A., et al. Optical properties of human skin, subcutaneous and muscous tissues in the wavelength range from 400 t2000 nm. Journal of Physics D-Applied Physics. 2005;38(15):2543-55.

73. Kondepati VR, Heise HM, Backhaus J. Recent applications of near-infrared spectroscopy in cancer diagnosis and therapy. Anal Bioanal Chem. 2008;390(1):125-39.

74. Tuchin VV. Tissue Optics: Light Scattering Methods and Instruments for Medical Diagnosis SPIE Publications; 2014. 882 p.

75. Barrington MJ, Snyder GL. Neurologic complications of regional anesthesia. Curr Opin Anaesthesiol. 2011;24(5):554-60. 



\section{Chapter \\ What is the best way to analyze optical spectra with the STS \\ system?}

Nerve detection with optical spectroscopy for regional anesthesia procedures

Benno H. W. Hendriks, Andrea J. R. Balthasar, Gerald W. Lucassen, Marjolein van der Voort, Manfred Mueller, Vishnu V. Pully, Torre M. Bydlon, Christian Reich, Arnold T. M. H. van Keersop, Jeroen Kortsmit, Gerrit C. Langhout, Geert-Jan van Geffen J Transl Med 2015;13:380 


\section{Abstract}

\section{Background}

Regional anesthesia has several advantages over general anesthesia but requires accurate needle placement to be effective. To achieve accurate placement, a needle equipped with optical fibers that allows tissue discrimination at the needle tip based on optical spectroscopy is proposed. This study investigates the sensitivity and specificity with which this optical needle can discriminate nerves from the surrounding tissues making use of different classification methods.

\section{Methods}

Diffuse reflectance spectra were acquired from 1563 different locations from 19 human cadavers in the wavelength range of 400-1710 nm; measured tissue types included fascicular tissue of the nerve, muscle, sliding fat and subcutaneous fat. Physiological parameters of the tissues were derived from the measured spectra and part of the data was directly compared to histology. Various classification methods were then applied to the derived parameter dataset to determine the accuracy with which fascicular tissue of the nerve can be discriminated from the surrounding tissues.

\section{Results}

From the parameters determined from the measured spectra of the various tissues surrounding the nerve, fat content, blood content, beta-carotene content and scattering were most distinctive when comparing fascicular and non-fascicular tissue. Support Vector Machine classification with a combination of feature selections performed best in discriminating fascicular nerve tissue from the surrounding tissues with a sensitivity and specificity around $90 \%$.

\section{Conclusions}

This study showed that spectral tissue sensing, based on diffuse reflectance spectroscopy at the needle tip, is a promising technique to discriminate fascicular tissue of the nerve from the surrounding tissues. The technique may therefore improve accurate needle placement near the nerve which is necessary for effective nerve blocks in regional anesthesia. 


\section{Background}

Regional anesthesia has shown advantages over general anesthesia; lower odds of inpatient mortality and pulmonary complications during hip repair, ${ }^{1}$ faster return of bowel function $^{2}$ as well as a lower risk for delirium and nausea have been reported. Current disadvantages of regional anesthesia are the unpredictable onset time of the block as well as the risk of a failed block and, although rare, the risk for complications caused by injection of local anesthetics into critical structures such as blood vessels or nerve fascicles (see Figure 2.1). ${ }^{3,4}$ Accurate needle placement with respect to the various structures is therefore important. ${ }^{5}$

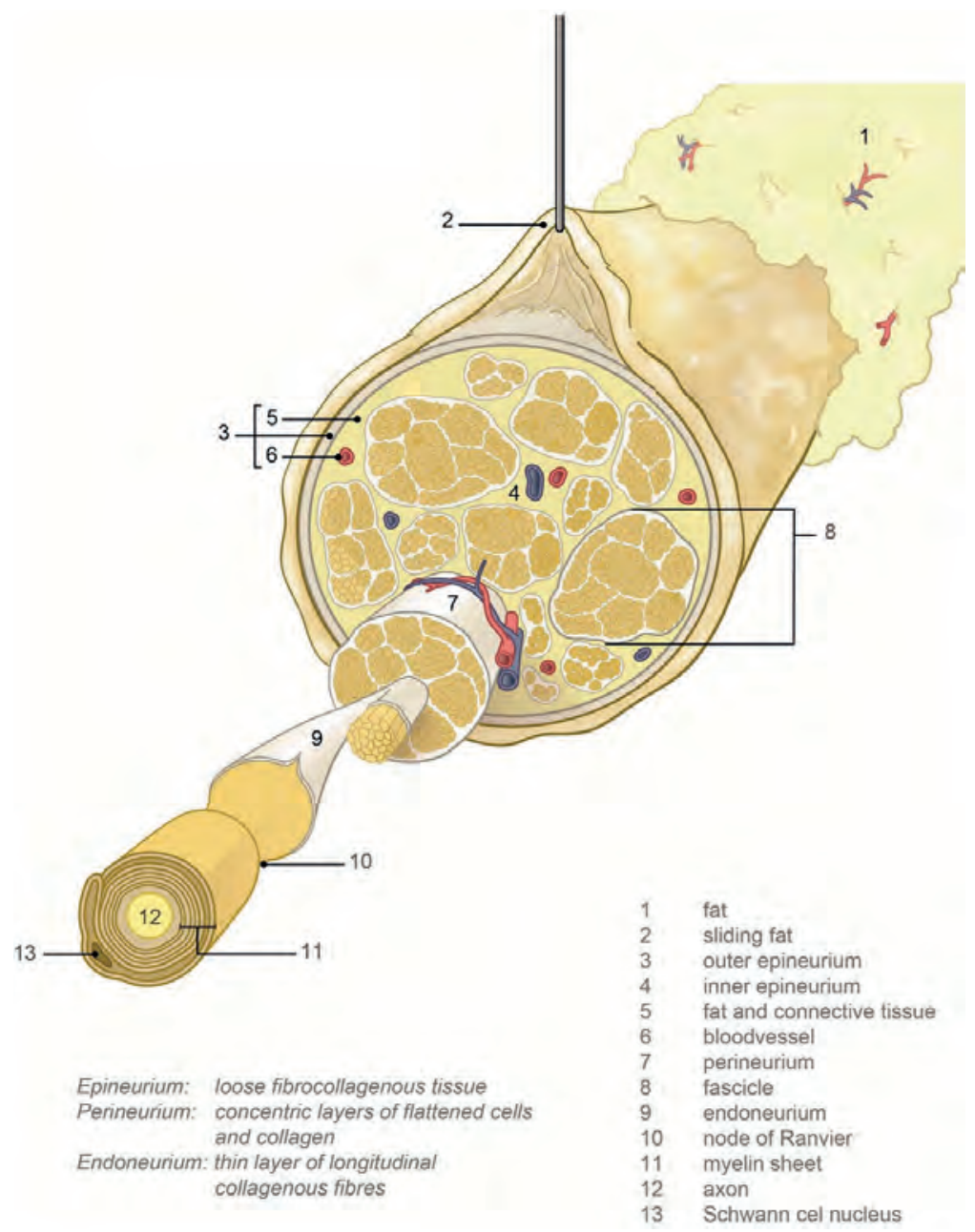

Figure 2.1 Schematic drawing of the nerve and the surrounding tissues. 
Techniques like paresthesia, electrical nerve stimulation and ultrasound guidance have been introduced to improve needle placement. The elicitation of paresthesia has shown a sensitivity of only $40 \%$ for nerve detection while for nerve stimulation up to $75 \%$ sensitivity has been reported. ${ }^{6,7}$ However, the stimulation threshold does not give an exact indication of the distance between the needle and nerve. Ultrasound (US) guidance for the performance of peripheral nerve blocks improves the quality of regional anesthesia because nerve structures can be seen as multiple rounded hypoechoic areas in a hyperechoic background or vice versa ${ }^{8}$ and the spread of local anesthetic is observed upon injection. However, the quality of the ultrasound guidance is user- and patientdependent, requires sufficient training, and additionally ultrasound imaging has technical limitations. For example, as the US frequency decreases, degradation in the image resolution occurs. ${ }^{9}$

Diffuse optical spectroscopy is a technique which sends white light into tissue and measures the reflected light. The reflected light has a specific spectral distribution due to the absorption and scattering of photons interacting with the tissue. Since these changes are determined by the tissue composition, the technique provides tissue discrimination. By incorporating optical fibers into needles, a method has been developed that can provide real-time tissue feedback at the tip of the needle. This spectral tissue sensing (STS) method is complementary to existing techniques that do not have this functionality. Therefore, STS could improve needle placement in regional anesthesia for accurate deposition of anesthetics. Recently, several studies have been performed aiming at nerve discrimination based on diffuse optical spectroscopy. ${ }^{10-12}$ In the study of Brynolf et al. ${ }^{10}$ the optical detection of the brachial plexus was studied in in vivo swine. While promising results were presented, the study was limited to detecting a "nerve target region" and performed on only two swine. Balthasar et al. ${ }^{11}$ investigated optical detection of peripheral nerves in 20 patients. Subcutaneous fat, muscle and a nerve target region were the tissue types included and detected in the study. The results indicated that the transitions from muscle to nerve target region could be identified reliably. However, the question of nerve proximity remained unanswered. Differentiation between nerve and adipose tissue with optical spectroscopy was investigated in thyroid and parathyroid surgery by Schols et al. ${ }^{12}$ Discrimination between nerve and adipose tissue was possible especially when measuring the spectra in the near-infrared region. However, due to the limited dataset of only including adipose tissue and nerve, no definitive conclusions could be drawn from this data regarding needle placement for regional anesthesia where the needle also traverses subcutaneous fat and muscle tissue.

In this study, it was investigated whether accurate needle placement near nerve structures of the cervical nerves (C-roots) and the median nerve of human cadavers was possible when guided by STS to facilitate needle placement for regional anesthesia. Furthermore, to investigate whether a detection algorithm based on fascicular tissue of the cervical nerve can be generalized to other nerves, spectra measured at different 
locations near the median nerve was validated on a model trained on the spectra acquired from the cervical nerve area.

\section{Methods}

\section{Human specimens}

Human tissue measurements were performed at the Anatomy department of the Radboud University Medical Center Nijmegen. In this study STS measurements were performed in 19 human cadavers, 11 male and 8 female with an age range of 65-92 years (mean age 80.4 years). A handwritten and signed codicil from the donor, as required by the Dutch law for scientific research and education is kept at the Department of Anatomy of the Radboud University Nijmegen, Nijmegen, The Netherlands.

The human cadavers were one-time frozen and selected to have no neurological abnormalities or diseased tissue near the intended measurement sites. In each cadaver the cervical area (from interscalene region to proximal until the neuroforamen) and forearm area (medial side of the mid forearm) was dissected to expose the nerves and surrounding tissues. In all human cadavers cervical nerves and surrounding tissues were measured and in ten of them the median nerves with surrounding tissues were also measured.

\section{Cervical nerve, median nerve and surrounding tissue anatomy}

In general after skin perforation, when advancing the needle towards the nerve a layer of subcutaneous fat, muscle tissue and the fat surrounding the nerve (sliding fat) will be traversed (see Figure 2.1). Sliding fat in our case is defined as fat surrounding the entire nerve bundle; fat outside the outer epineurium. In Figure 2.2 the histology of subcutaneous fat, muscle, the cervical nerve and the median nerve is shown. The cervical nerve at the proximal side is mostly mono-fascicular branching into multiple fascicular bundles at the distal side. The fascicular bundles are surrounded by loose collagenous tissue and adipose tissue of the epineurium. ${ }^{13}$ The median nerve is already multi fascicular at the proximal side, with the number of fascicles increasing towards the distal side. Subcutaneous fat shows a high amount of adipose cells with some connective tissue while muscle tissue is mostly composed of muscle fibers. 

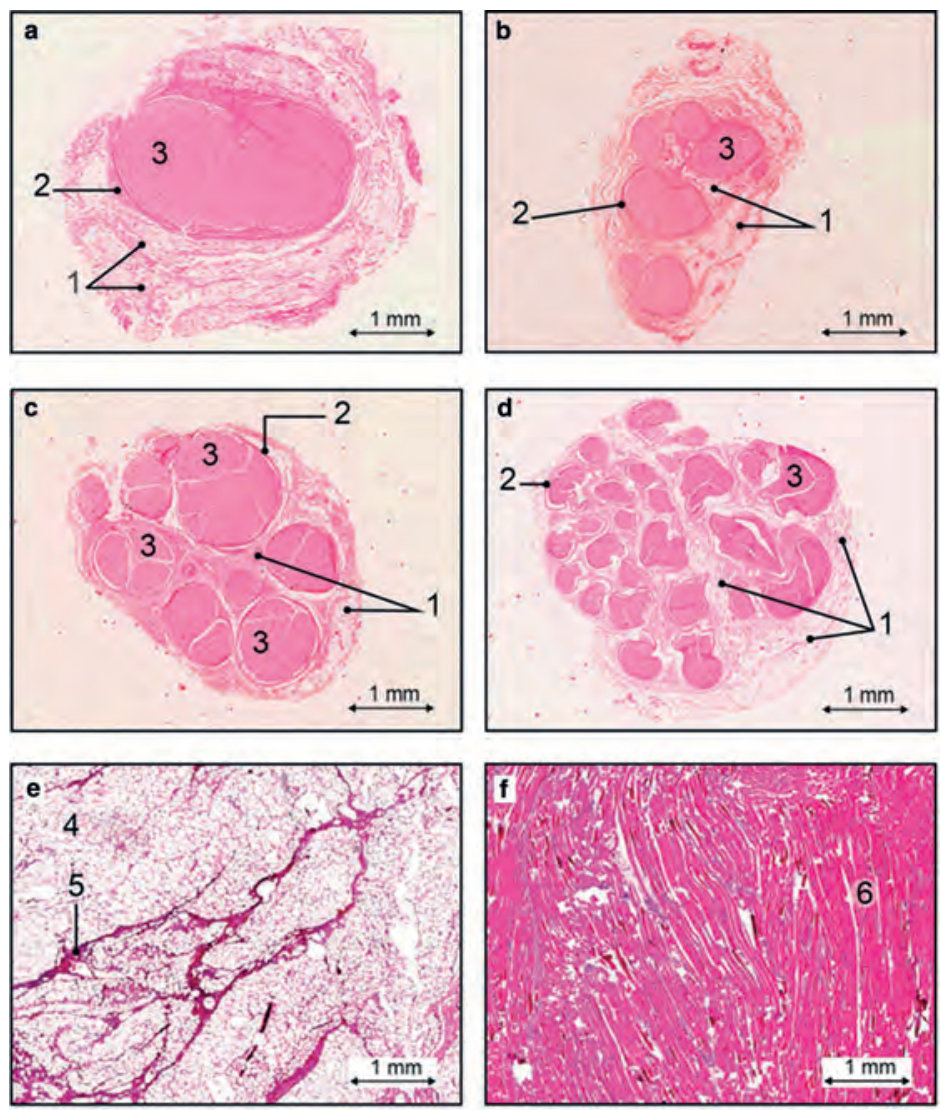

1 epineurium: connective tissue, bloodvessels, fat

2 perineurium: concentric layers of flattened cells and collagen

3 nerve fascicle

4 connective tissue, fat

5 collagenous fibres

6 muscle fascicles

Figure 2.2 Histology slides of the nerves and surrounding tissues studied: cross section of the cervical nerve (C6-root) with some surrounding areolar tissue proximal (a) and distal (b); cross section of the median nerve proximal (c) and distal (d), subcutaneous fat (e) and muscle (f).

\section{Instrumentation}

Ex vivo diffuse reflectance spectra were acquired using a portable spectroscopic system as illustrated in Figure 2.3. A tungsten halogen broadband light source with an integrated shutter (Avantes HAL-S) was used as an emitting source. Delivery of light to the tissue and its collection were achieved with a $1.0 \mathrm{~mm}$ diameter fiber-optic probe with the distal end polished at an angle of 30 degrees. The probe consisted of two $200-\mu \mathrm{m}$ core 
diameter optical fibers with a tip to tip distance of $0.85 \mathrm{~mm}$. The illumination fiber was connected to the light source and the collection fiber was connected to a spectrometer with a silicon detector (Horiba, S318-2 VIS) and a spectrometer with an InGaAs detector (Horiba-S330-2 NIR), via a 50-50\% fiber splitter to divide the collected light over the two detectors.

The diffuse optical spectra were calibrated by subtracting a background measurement acquired after each tissue measurement and divided by the response of a white reflectance standard (Spectralon) with known reflectivity.

Acquisition time per spectrum was $1 \mathrm{~s}$ including background measurement. At each measurement location typically 10 spectra were acquired that were averaged to form one data point in the database.
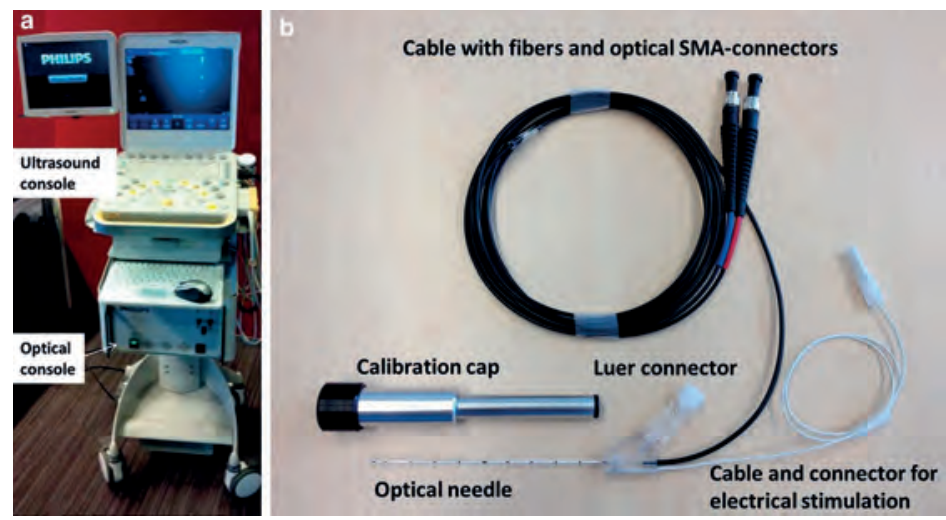

Figure 2.3 Picture of the optical console (left) and the optical needle (right) used in the measurements.

\section{Data acquisition}

In this study STS measurements were performed on 19 human cadavers. A database was compiled of spectra measured at 1274 different locations on and near nerve roots as well as at 289 different locations on and near the median nerve. Spectra (400-1710 nm) were acquired from subcutaneous fat, muscle, sliding fat, and fascicular tissue of the nerve. Two experienced anesthesiologists, with the assistance of an anatomist, positioned the optical needle on the exposed tissues. In all cases we ensured that the optical fibers of the needle made good contact with the tissue to avoid artifacts due to air voids. Because the needle was held by hand some slight movements could have occurred but analysis of several measurements taken at one location did not show significant variations in the spectra. To avoid that a mixture of the tissue types was measured we made sure that the tissue layer was thicker than $2 \mathrm{~mm}$. Especially for sliding fat, the optical needle was placed such that no nerve structure was in the direction of the light beam. 


\section{Feature extraction methods}

All data processing was performed using software developed in-house based on MATLAB (Mathworks Inc.) and its Statistics toolbox. Where third party toolboxes were used, they are mentioned below.

With a resolution of $1 \mathrm{~nm}$ each measured spectrum consists of 1311 data points. To avoid over-fitting, the number of data points must be translated into features, with the number of features considerably lower than the number of spectra measured. Several methods to reduce the number of data points into relevant features were considered. In particular we considered methods with limited dependence on absolute intensities since the absolute intensity of the spectra may fluctuate significantly due to small variations in calibration conditions, the state of the probe, or even the bending radius of the fibers.

The first feature extraction method was a fitting method which decomposes a spectrum into 12 fit parameters i.e. features. The second method was to perform a standard principal component analysis (PCA) and use the most important principal components as features. $^{14}$

\section{Physiological fit parameters}

Spectral data modelling can relate the measured spectra to underlying physiological parameters like blood and fat concentrations. The spectra were fit with a modified version of the model developed by Farrell et al.. ${ }^{15}$ This model estimates the absorption coefficient $\mu_{\mathrm{a}}(\lambda)$ and the reduced scattering coefficient $\mu_{\mathrm{s}^{\prime}}(\lambda)$ expressed in $\mathrm{cm}^{-1}$.

To determine the chromophore concentrations present in the tissue, the fiber distance between the emitting and collecting fibers as well as the wavelength-dependent absorption coefficients of these chromophores need to be known. ${ }^{16-18}$ The chromophores that were taken into account in this study were the blood related absorbers deoxygenated-hemoglobin ( $\mathrm{Hb}$ ) and oxygenated-hemoglobin ( $\mathrm{HbO} 2$ ), $\beta$-carotene, fat, water and collagen. The blood oxygen saturation (StO2) is defined as the fraction of hemoglobin that is oxygenated i.e. $\mathrm{HbO} 2 /(\mathrm{Hb}+\mathrm{HbO} 2)$. The fat-to-water and fat fraction $F /(W+F)$ is defined as fat content divided by the water and fat content of the tissue. For the scattering the empirical model described by

$$
\mu_{s^{\prime}}(\lambda)=\operatorname{S} 800\left[\text { Fmie }\left(\frac{\lambda}{\lambda_{0}}\right)^{-b}+(1-\text { Fmie })\left(\frac{\lambda}{\lambda_{0}}\right)^{-4}\right]
$$

is used where $\lambda_{0}=800 \mathrm{~nm}$ corresponds to a wavelength normalization value, $\mathrm{S} 800$ is the reduced scattering amplitude at $\lambda_{0}$, the Mie scattering slope is $b$, and Fmie denotes the Mie-to-total reduced scattering fraction assuming that Mie and Rayleigh scattering are the two dominant types of scattering in tissue. 


\section{Wavelength dependent features}

Each fit parameter and principal component relates to the full wavelength range of the spectrum, so we also developed a method whereby each feature relates to a certain wavelength range. In this third method the spectrum was divided into equally sized wavelength segments. For each segment a feature was calculated according to how the spectrum slopes in the segment as compared to the averaged spectra. To avoid the influence of intensity variations this was performed on normalized spectra. To determine the difference from the average spectra, the intensities were scaled for each wavelength, so that the average intensity was 0 and the standard deviation was 1 . The scaled intensities were then added to obtain a scalar feature. Segment values are strongly positive when a spectrum slopes significantly more upward than the average, strongly negative when it slopes more downwards, and around zero when it behaves averagely or when the individual spectrum crosses the average spectrum.

This segment feature extraction method was considered as representative for the many other methods that can be used to describe the (relative) behavior of a curve.

\section{Classification methods}

Different classification methods were investigated to discriminate between fascicular tissue of the nerve and nonfascicular tissue (muscle, subcutaneous and sliding fat). These included: partial least squares discriminant analysis (PLS-DA), support vector machine (SVM) and classification and regression trees (CART) analysis.

\section{Partial least squares discriminant analysis (PLS-DA)}

Partial least-squares (PLS) ${ }^{19}$ analysis is a regression method to find a linear relationship between a response variable $Y$ (tissue type class) and the independent variable $X$ (spectra). PLS-DA is widely used for analysis of spectra. The method is based on finding a number of principal components that represent as much of the variance in $\mathrm{X}$ as is possible. PLS-DA selects the principal components which are most relevant to the response variable Y. Therefore, PLS-DA acts directly on the spectra without the need for prior feature extraction. The PLS model is generated using a training data set. A discriminant analysis (DA) method is subsequently performed to obtain thresholds for discriminating the different responses (i.e. tissue classes). Prediction of class (tissue type) on the remaining data (the validation data set) is obtained by comparing the predicted PLS scores with the DA thresholds. The measured tissue type is assigned to one of the two predefined tissue classes depending on the PLS scores. The PLS-DA algorithm scripts used PLS Toolbox 6.2 (Eigenvector Research, Inc, Wenatchee, WA). 


\section{Support vector machine (SVM)}

Support vector machines $(S V M)^{20}$ divide the feature space with a hyperplane that separates the two classes. In case full separation is not possible, a penalty parameter determines the behavior of the boundary. We used LIBSVM (http://www.csie.ntu.edu.tw/ cjlin/libsvm/) with a radial Kernel and standard parameters. Before using SVM, all features were scaled to a mean of 0 and a standard deviation of 1 .

\section{Classification and regression trees (CART) analysis}

The classification and regression tree (CART) is based on a binary recursive partitioning algorithm. It starts from a central node that discriminates the two classes based on the best discriminating feature. From this node, daughter partial trees are generated and other features or the same are used for further splits. The purity of each node is assessed with the Gini's maximization index algorithm which corresponds to unity minus the sum of square of the proportions of target classes at a specific node. ${ }^{21}$

\section{Statistical analysis}

All classification methods used a training data set to create a classification model. A second, validation data set was then used to calculate accuracy, sensitivity and specificity for the model based on the confusion matrix of the validation data. Sensitivity and specificity are complementary (one can improve sensitivity at the cost of specificity and vice versa) so Matthews correlation coefficient (MCC) was used as a scalar measure for the quality of classification. MCC is given by ${ }^{22}$ :

$$
\mathrm{MCC}=\frac{\mathrm{TP} \times \mathrm{TN}-\mathrm{FP} \times \mathrm{FN}}{\sqrt{(\mathrm{TP}+\mathrm{FP})(\mathrm{TP}+\mathrm{FN})(\mathrm{TN}+\mathrm{FP})(\mathrm{TN}+\mathrm{FN})}}
$$

where TP are the true positives, TN the true negatives, FP the false positives and FN the false negatives. Here, fascicular tissue of the nerve was considered as a "positive" and surrounding non-fascicular tissues as a "negative". The value of MCC is between -1 and 1 , where 1 represents a perfect prediction, 0 no better than random and -1 total disagreement between prediction and actual state.

For the cervical nerve dataset a leave-one-specimen-out cross-validation approach was used, meaning the classification models were trained on the spectra from all but one cadaver and the validation was done on the spectra from the cadaver left out. This was repeated until all cadavers had been left out once, with the confusion matrices being added up.

The datasets acquired for the median nerve and surrounding tissue were classified by training the algorithms on the cervical nerve and surrounding tissues database only 
and then applying the model to the datasets collected in the area of the median nerve. This was to show how the classification algorithms work on truly independent data.

\section{Results}

\section{Tissue spectra}

In total spectra were collected from 1274 different locations in the neck area of 19 human cadavers resulting in a dataset consisting of: 702 fascicular measurements from the cervical nerves, 223 muscle, 164 sliding fat and 185 subcutaneous fat. In addition, spectra were collected from 289 different locations near the median nerve: 107 median nerve, 76 muscle, 46 sliding fat and 60 subcutaneous fat tissues.

In Figure 2.4 the average spectra are shown measured on subcutaneous fat, muscle, sliding fat and fascicular tissue of the cervical nerve (nerve tissue). To determine the physiological differences between the tissues in Figure 2.5 boxplots of the most relevant parameters determined by the fit model are shown. A blood content of $100 \%$ is equivalent to $150 \mathrm{~g}$ hemoglobin/liter. The blood content in muscle tissue is found to be higher than nerve, and is lowest in subcutaneous and sliding fat which is in line with expectations based on the diffuse reflectance spectrum shown in Figure 2.4, since the amount of blood is linked to the absorption dip near 550-600 nm. Blood oxygen saturation was low for muscle tissue and comparable for the other tissues. Adipose rich tissues (subcutaneous and sliding fat), show high fat content, while for nerve and muscle lower values were found. This is also observed in the typical spectra where higher fat values result in a more pronounced absorption dip around $1210 \mathrm{~nm}$. Beta-carotene, absorbing near 500 $\mathrm{nm}$ causing a yellow appearance of tissue, shows a similar behavior to the fat content. Results for the collagen content estimated from the fit model show higher values in muscle and nerve. For the scattering related parameters, the contribution of the Mie scattering (Fmie) and the Mie slope parameter (b) show largest differences between the four tissues. 


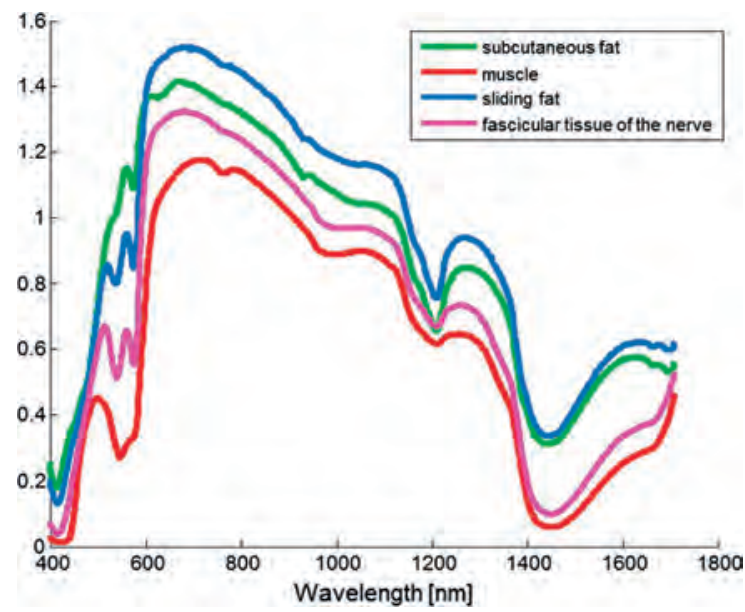

Figure 2.4 Averaged spectra measured for the four tissue classes.
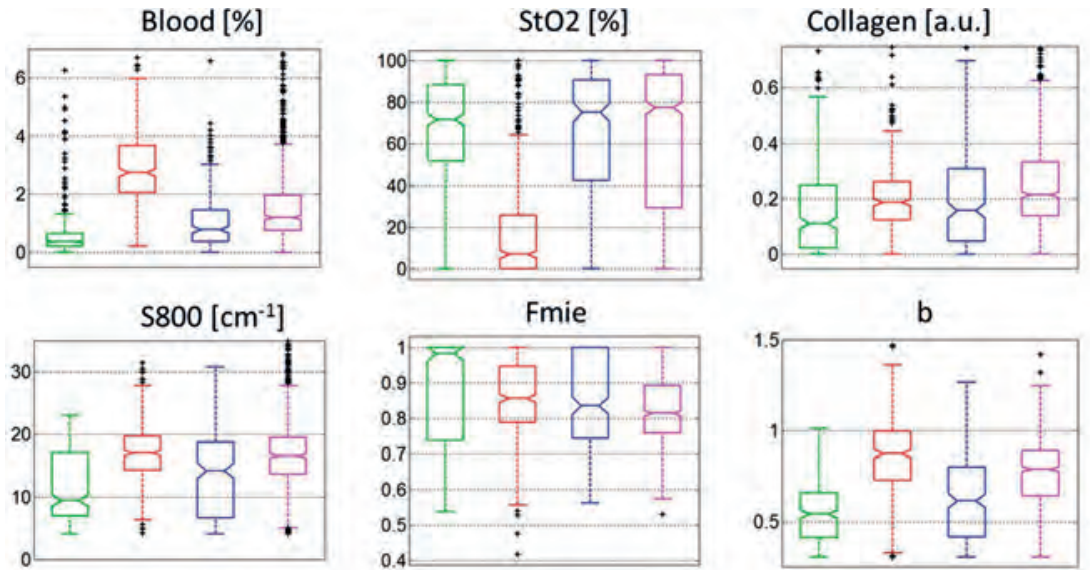

b
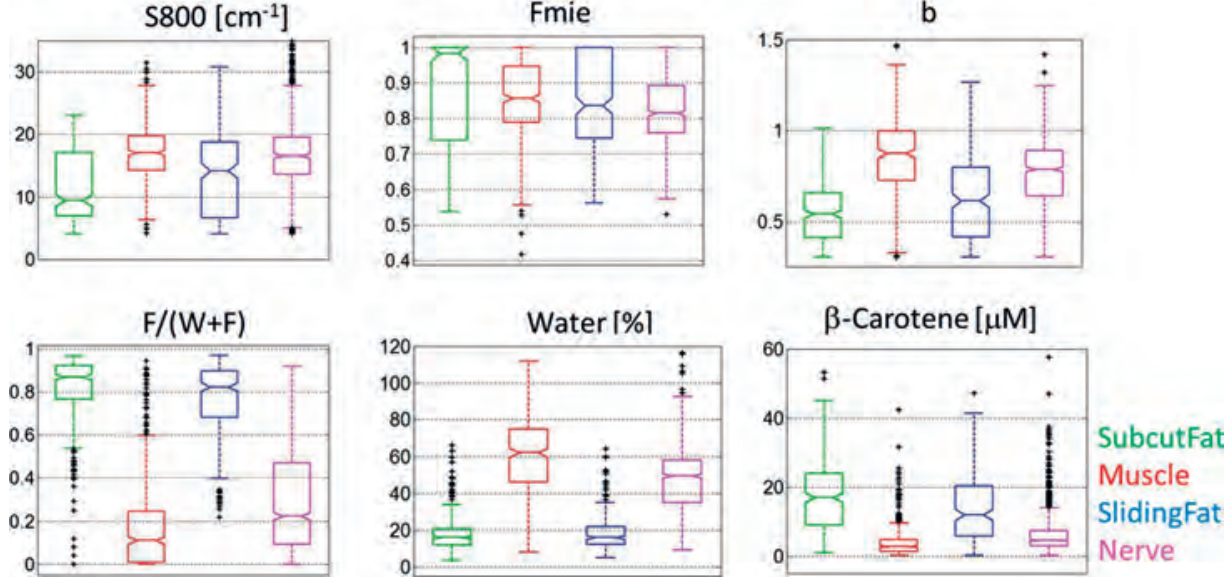

Figure 2.5 Boxplots of the various parameters derived from the fit model. Note the plots have been adjusted to maximize visualization of the boxes and whiskers; therefore, not all outliers are shown. 


\section{Tissue classification}

In Table 2.1, the classification results for fascicular discrimination from the surrounding non-fascicular tissues (subcutaneous fat, muscle and sliding fat) according to the methods considered in this paper are summarized. For the SVM classification, the different feature extraction methods (Fit, PCA and Segments) as well as a combined feature that combines all three were considered. The classification results show that accurate discrimination of nerve tissue is possible, independent of the classification method used. The highest achieved sensitivity and specificity were both around $90 \%$.

The number of principal components and the length of the segments were optimized in a coarse way. For SVM, 30-40 principal components and short segments with a length between 10 and $20 \mathrm{~nm}$ resulted in the best performance. The table contains the values for 30 principal components and $20 \mathrm{~nm}$ segments. As mentioned before, a leaveone-specimen-out cross-validation approach was applied for this analysis.

Table 2.1 Classification results according to SVM, PLS-DA and CART for discrimination of fascicular tissue of the nerve from the surrounding tissues.

\begin{tabular}{llllllllllll}
\hline $\begin{array}{l}\text { Classification } \\
\text { method }\end{array}$ & $\begin{array}{l}\text { Feature } \\
\text { selection }\end{array}$ & MCC & ACC & SENS (\%) & SPEC (\%) PPV & NPV & TP & FN & FP & TN \\
\hline SVM & Fit & 0.711 & 0.854 & 82.6 & 88.8 & 0.901 & 0.806 & 580 & 122 & 64 & 508 \\
SVM & PCA & 0.793 & 0.897 & 89.9 & 89.5 & 0.913 & 0.878 & 631 & 71 & 60 & 512 \\
SVM & Segments & 0.779 & 0.890 & 88.6 & 89.5 & 0.912 & 0.865 & 622 & 80 & 60 & 512 \\
SVM & Combined & 0.826 & 0.914 & 91.3 & 91.4 & 0.929 & 0.896 & 641 & 61 & 49 & 523 \\
PLSDA & 10PC's & 0.814 & 0.907 & 92.5 & 89.5 & 0.864 & 0.943 & 494 & 40 & 78 & 662 \\
CART & Fit & 0.615 & 0.808 & 81.2 & 80.4 & 0.836 & 0.777 & 570 & 132 & 112 & 460 \\
\hline
\end{tabular}

For SVM different feature selection methods are used: fit parameters, PCA, segments and a combination of the last three. For PLSDA, 10 principal components have been used (10PC's). For the CART analysis, the fit parameters have been used as features.

Matthews correlation coefficient (MCC see Eq. 2 text), accuracy $(A C C=[T P+T N] /[T P+F N+F P+T N])$, sensitivity $(S E N S=T P /[T P+F N])$, specificity $(S P E C=T N /[F P+T N])$, positive predictive value $(P P V=T P /[T P+F P])$, negative predictive value (NPV = TN/[TN + FN]), true positive $(T P)$, false negative (FN), false positive (FP), true negative (TN).

To investigate the validity of the classification when applying the models trained on the data from the neck area to classify spectra from tissues in other areas of the body, the above analysis was repeated. In Table 2.2, the classification results are listed for median nerve discrimination from the surrounding tissues when the classification algorithm was trained on data collected from C-root nerves and surrounding tissues in the neck area only. Overall, the results of that analysis show somewhat lower sensitivity and specificity. Nonetheless, sensitivity and specificity above $85 \%$ were achieved with most methods. 
Table 2.2 Classification results according to SVM, PLS-DA and CART for discrimination of fascicular tissue of the median nerve from the surrounding tissues validation data collected in the forearm area) when training the various methods using data collected in the neck area.

\begin{tabular}{|c|c|c|c|c|c|c|c|c|c|c|c|}
\hline $\begin{array}{l}\text { Classification } \\
\text { method }\end{array}$ & $\begin{array}{l}\text { Feature } \\
\text { selection }\end{array}$ & MCC & ACC & SENS (\%) & SPEC (\%) & PPV & NPV & TP & FN & FP & TN \\
\hline SVM & Fit & 0.683 & 0.848 & 85.0 & 84.6 & 0.765 & 0.906 & 91 & 16 & 28 & 154 \\
\hline SVM & PCA & 0.693 & 0.855 & 83.2 & 86.8 & 0.788 & 0.898 & 89 & 18 & 24 & 158 \\
\hline SVM & Segments & 0.717 & 0.862 & 88.8 & 84.6 & 0.772 & 0.928 & 95 & 12 & 28 & 154 \\
\hline SVM & Combined & 0.751 & 0.882 & 86.9 & 89.0 & 0.823 & 0.920 & 93 & 14 & 20 & 162 \\
\hline PLSDA & $10 P C^{\prime} s$ & 0.580 & 0.789 & 84.1 & 75.8 & 0.672 & 0.890 & 90 & 17 & 44 & 138 \\
\hline CART & Fit & 0.442 & 0.730 & 71.0 & 74.2 & 0.618 & 0.813 & 76 & 31 & 47 & 135 \\
\hline
\end{tabular}

\section{Discussion and conclusion}

In this study the accuracy with which STS is able to discriminate nerve tissue from surrounding tissues during needle interventions for regional anesthesia was investigated. Of four tissue types spectra were collected from 19 human cadavers at 1563 different locations: 1274 spectra acquired from the cervical nerve area and 289 spectra from the median nerve area. Various classification algorithms were applied to the dataset showing that fascicular tissue of the nerve can be discriminated from surrounding tissues with sensitivity and specificity around $90 \%$. These results were based on the largest dataset collected to date in nerve and surrounding tissues and confirm earlier reports on nerve discrimination with smaller data sets. ${ }^{10-12}$

The histology slides show that the nerves studied consisted mainly of fascicular bundles enclosed by loose collagenous tissue and adipose tissue of the epineurium. The outer-epineurium is surrounded by a layer of sliding fat with variable thickness. The boxplots of the physiological parameters obtained by fitting the spectra show a significant difference between fat content for tissues such as subcutaneous fat and sliding fat compared to muscle and nerve tissues. The same holds for beta-carotene which is present in fatty tissues. ${ }^{18}$ The blood content in muscle tissue is on average higher while the blood oxygen saturation herein is lower than in the surrounding tissues. Furthermore, from the parameters related to scattering ( 8000 , Fmie and b), the Mie slope b seems to differ most between the tissues. Collagen content shows higher values in muscle and nerve which is in line with the fact that those tissues are richer in connective tissue compared to the other tissues investigated. ${ }^{13}$ In general, there is a significant variation in the parameters determined within each tissue, and outliers are present (indicated with ' + '). Despite these variations and outliers, the nerve tissue can be discriminated with high accuracy from the surrounding tissues for this large data set.

The spectra were measured in one-time frozen human cadavers, where the tissues were exposed so that the physician was able to validate the tissue type measured visually. Physiological parameters that are known to change from in vivo to ex vivo are the 
blood content and blood oxygen saturation. The settling of blood (livor mortis ${ }^{23}$ ) will affect the local blood content while the blood oxygen saturation in the exposed tissue is likely to be different from living tissue because of no blood circulation as well as the uptake of oxygen directly from the air. In what way this affects the nerve discrimination when performing the measurements in vivo is part of future investigation.

Of the various dimension reduction methods the fit method resulted in the lowest MCC. The fit method reduces the spectral information into 12 independent parameters thereby losing relevant information for discriminating nerves. Additionally the fit method, based on the analytical model derived by Farrell et al., ${ }^{15}$ assumes the tissue to be homogenous. The histological results, however, show that within the optical probing volume of $1 \mathrm{~mm} 3$ this assumption is not always fulfilled, especially near the nerve that has a more layered structure. Despite these disadvantages, the fit method provides the opportunity to link observed features in the spectra to the histological and physiological properties of the studied tissues. Trends in the boxplots of Figure 2.5 can be linked to the histology shown in Figure 2.2.

There is significant variation present in the measured spectra per tissue type. As a result, discrimination of the various tissues requires taking multiple parameters into account, in our case up to 40 spectral features. The danger of over fitting the data is prevented by our large spectral database collected in 19 specimens. From the classification methods, SVM performed best. SVM may be able to perform well on multidimensional parameter spaces because it can ignore features that do not significantly contribute to the discrimination. The fit dimension reduction allows easy interpretation of the results since the input parameters for classification are used whereas other methods post-process the parameters into scores that might not be intuitively related to the input parameters. From the fit parameters, the scattering parameters, the fat and water content as well as blood oxygen saturation contributed most to the fascicular tissue of the nerve discrimination. In general, the various classification methods resulted in similar accuracies, demonstrating the robustness of STS for tissue discrimination. This robustness allows flexibility in classification algorithm design when developing tissue discrimination based on STS into a product.

The cervical nerves as well as the median nerves investigated in this study are mostly composed of fascicular tissue with a limited amount of epineurium that consists of irregular connective tissue and adipocytes. This composition is known to be different in various nerves and changes when going from proximal to distal locations of the nerve. ${ }^{24}$ When training the classification algorithm on the cervical nerve area only and testing it on the median nerve area, the sensitivity and specificity for discrimination were somewhat lower than for cervical nerve discrimination alone (see Tables 2.1, 2.2). Apparently, the variation in nerve tissue composition observed in the histology shown in Figure 2.2 requires fine tuning of the classification algorithm. To what extent this variation in composition affects the nerve discrimination for nerves at other locations in the body has to be further investigated. 
To ensure that the correct tissue type was measured, two experienced anesthesiologists with the assistance of an anatomist positioned the optical needle on the exposed tissues. The exposed setting with visual confirmation was considered to be the best gold standard, as opposed to ultrasound where the needle tip/nerve visualization is prone to errors. Special attention was given to measure pure tissue types which was sometimes challenging for thin structures such as sliding fat and when placing the needle on a nerve that could slide away. Despite these difficulties, the errors that might be introduced by these measurement inaccuracies will have limited influence on the final classification accuracy due to the large dataset we acquired.

Comparing STS (sensitivity around $90 \%$ ) with other needle guidance techniques shows a significant improvement in nerve detection compared to paresthesia (sensitivity of $40 \%$ ) [6] and nerve stimulation (sensitivity up to $75 \%$ ). ${ }^{7}$ Another advantage of the STS method is that the detection distance (determined by the distance between fiber ends) is on the order of a millimeter, allowing more accurate placement near the nerve compared to the other methods where, even when the needle detects nerve, the exact distance is not well known. Both improvements compared to current practice may contribute to safer loco-regional blocks in regional anesthesia.

From a clinical point of view, the most vulnerable and important structure of the nerve is the nerve fascicle itself. During the administration of loco-regional anesthesia, nerve-fascicles can be damaged directly or manipulation of surrounding tissue during the procedure, or indirect trauma due to ischemia by damaging the supplying blood vessel, can occur. The cause of damage, but also the site of damage, impacts the consequences. Proximal damage causes more neurological deficit than distal damage. Also, the proximal part of the nerve is more prone to damage than the distal area. In an ideal situation, the nerves should be detected right on time, the injection of fluids should not cause damage, the spread of the anesthetic fluid is perfect and the onset time of the block is fast. Several ideal needle placements are theoretically possible. One could be just outside the nervestructure, e.g. outer epineurium. In case of proximal nerve roots or cases of nearly mono-fascicular nerves this would be achievable. Another one could be inside the epineurium, e.g. intra-epineurally or parafascicular, but not intrafascicular. This needle position may be an option in more peripheral nerves with a lot of connective tissue and fat inside the epineurium, where the risk of damaging a fascicle is probably lower.

This study shows that employing STS at the tip of the needle is a promising technique that may improve accurate needle placement near nerve structures in regional anesthesia. In particular penetration of the needle into the nerve-fascicle could be avoided. However, before this can be implemented in real clinical practice, further validation is required where the technique is investigated in an in vivo setting where the different tissue structures are approached percutaneously. This will be part of future investigations. 


\section{References}

1. Neuman MD, Silber JH, Elkassabany NM, Ludwig JM, Fleisher LA. Comparative effectiveness of regional versus general anesthesia for hip fracture surgery in adults. Anesthesiology. 2012;117:72-92.

2. Breen P, Park KW. General anesthesia versus regional anesthesia. Int Anesthesiol Clin. 2002;40:61-71.

3. Auroy Y, Narchi P, Messiah A, Litt L, Rouvier B, Samii K. Serious complications related to regional anesthesia: results of a prospective survey in France. Anesthesiology. 1997;87:479-86.

4. Auroy Y, Benhamou D, Bargues L, Ecoffey C, Falissard B, Mercier FJ, Bouazziz H, Samii K. Major complications of regional anesthesia in France: the SOS Regional Anesthesia Hotline Service. Anesthesiology. 2002;97:1274-80.

5. Selander D, Brattsand R, Lundborg G, Nordborg C, Olson Y. local anesthetics: importance of mode of application, concentration and adrenaline for the appearance of nerve lesions: an experimental study of axonal degeneration and barrier damage after intrafascicular injection or topical application of bupivacaine (Marcah ${ }^{\circledast}$ ). Acta Anaesthesiol Scand. 1979;23:127-36.

6. Urmey WF, Stanton J. Inability to consistently elicit a motor response following sensory paresthesia during interscalene block administration. Anesthesiology. 2002;96:552-4.

7. Perlas A, Niazi A, McCartney C, Chan V, Xu D, Abbas S. The sensitivity of motor response to nerve stimulation and paresthesia for nerve localization as evaluated by ultrasound. Reg Anesth Pain Med. 2006;31:445-50.

8. Walker KJ, McGrattan K, Aas-Eng K, Smith AF. Ultrasound guidance for peripheral nerve blockade. Cochrane Database Syst Rev. 2009;4(4):CD006459. doi:10.1002/14651858.CD006459.pub2.

9. Silverstri E, Martinoli C, et al. Echotexture of peripheral nerves: correlation between US and histologic findings and criteria to differentiate tendons. Radiology. 1995;197:291-6.

10. Brynolf M, Sommer M, Desjardins AE, van der Voort M, Roggeveen S, Bierhoff W, Hendriks BHW, Rathmell JP, Kessels AGH, Söderman M, Holström B. Optical detection of the brachial plexus for peripheral nerve blocks: an in vivo swine study. Reg Anesth Pain Med. 2011;36:350-7.

11. Balthasar A, Desjardins AE, van der Voort M, Lucassen GW, Roggeveen S, Wang K, Bierhoff W, Kessels $A G$, van Kleef $M$, Sommer M. Optical detection of peripheral nerves: an in vivo human study. Reg Anesth Pain Med. 2012;37:277-82.

12. Schols RM, ter Laan M, Stassen LP, Bouvy ND, Amelink A, Wieringa FP, Alic L. Differentiation between nerve and adipose tissue using wide-band $(350-1830 \mathrm{~nm})$ in vivo diffuse reflectance spectroscopy. Lasers Surg Med. 2014;46:538-45.

13. Young B, Lowe JS, Stevens A, Heath JW. Wheater's functional histology, vol. 5th. London: Churchill Livingstone Elsevier; 2006.

14. Jolliffe IT. Principal component analysis. Series: Springer series in statistics, vol. 2nd. New York: Springer; 2002.

15. Farrell TJ, Patterson MS, Wilson B. A diffusion theory model of spatially resolved, steady-state diffuse reflectance for the noninvasive determination of tissue optical properties in vivo. Med Phys. 1992;19:879.

16. Nachabé R, Hendriks BHW, Desjardins AE, van der Voort M, van der Mark MB, Sterenborg HJCM. Estimation of lipid and water concentrations in scattering media with diffuse optical spectroscopy from 900 to 1600 nm. J Biomed Opt. 2010;15:037015.

17. Nachabé R, Hendriks BHW, Desjardins AE, van der Voort M, Sterenborg HJCM. Estimation of biological chromophores using diffuse optical spectroscopy: benefit of extending the UV-VIS wavelength range to include 1000-1600 nm. Biomed Optics Express. 2010;1:1432-42.

18. Bydlon TM, Nachabé R, Ramanujam N, Sterenborg HJCM, Hendriks BHW. Chromophore based analyses of steady-state diffuse reflectance spectroscopy: current status and perspectives for clinical adoption. J Biophotonics. 2014;8:9-24.

19. Barker M, Rayens W. Partial least squares of discrimination. J Chemom. 2003;17:166-73. 
20. Wu X, Kumar V, Quinlan JR, Gosh J, Yang Q, Motoda H, McLachlan GJ, Ng A, Liu B, Yu PS, Zhou Z-H, Steinbach M, Hand DJ, Steinberg D. Top ten algorithms in data mining. Knowl Inf Syst. 2008;14:1-37.

21. Breiman L. Classification and regression trees. Belmont: Wadsworth International group; 1984.

22. Matthews BW. Comparison of the predicted and observed secondary structure of T4 phage lysozyme. Biochimica et Biophysica Acta (BBA) - Protein. Structure. 1975;405:442-51.

23. Buchan MJ, Anderson GS. Time since death: a review of the current status of methods used in the later postmortem interval. Can Soc Forensic Sci J. 2001;34:1-22.

24. Moayeri N, Groen GJ. Differences in quantitative achitecture of sciatic nerve may explain differences in potential vulnerability to nerve injury, onset time, and minimum effective anesthetic volume. Anesthesiology. 2009;111:1128. 




\section{Chapter}

\section{Is it possible to detect intravascular needle position with the STS system?}

3A Is it possible to detect intravascular needle position with the STS system during nerve blocks?

3B: Is it possible to identify intra- and extravascular needle placement with the STS system? 



\section{Chapter}

\section{Is it possible to detect intravascular needle position with the STS system during nerve blocks?}

Optical detection of vascular penetration during nerve blocks: an in vivo human study

Andrea Balthasar, Adrien E. Desjardins, Marjolein van der Voort, Gerald W. Lucassen, Stefan Roggeveen, Ke Wang, Walter Bierhoff, Alfons G.H. Kessels, Micha Sommer, Maarten van Kleef Reg Anesth Pain Med 2012;37(1):3-7 


\section{Abstract}

\section{Background and objectives}

Complications resulting from vascular penetration during nerve blocks are rare but potentially devastating events that can occur despite meticulous technique. In this in vivo human pilot study, we investigated the potential for detecting vascular penetration with optical reflectance spectroscopy during blocks of the sympathetic chain and the communicating ramus at lumbar levels.

\section{Methods}

A custom-designed needle stylet with integrated optical fibers was used in combination with a commercial needle shaft. The needle stylet was connected to a console that delivered broadband light to tissue and spectrally resolved light that was scattered near the stylet tip. A total of 18 insertions were performed on 10 patients; testing for vascular penetration at the nerve target region was performed with aspiration and with radio-opaque contrast injections, visualized fluoroscopically. Optical absorption by hemoglobin was quantified with a blood parameter that was calculated from each spectrum. The blood parameter provided a measure of the difference between spectra acquired from the nerve target region and reference spectra acquired from blood extracted from a volunteer.

\section{Results}

In 2 insertions, vascular penetration was detected. Pronounced optical absorption by hemoglobin was observed to be associated with both of these events and absent in all other cases. The difference between the blood parameters obtained when vascular penetration was detected, and all other blood parameters were statistically significant $(P=0.006)$, with a diagnostic odds ratio of 35.4 (confidence interval, 2.21 to $\infty$ ).

\section{Conclusions}

The results from this study suggest that optical spectroscopy has the potential to detect intravascular needle placement, which may in turn increase the safety of nerve blocks. 


\section{Introduction}

Minimizing the risk of vascular complications is of critical importance during percutaneous injections for interventional pain management. Unintentional intravascular injections of local anesthetics and corticosteroids can contribute to poor procedure outcomes and can lead to neurologic and cardiovascular complications including quadriplegia, cortical blindness, stroke, and death. ${ }^{1}$ One mechanism of injury is ischemia caused by end-arteriolar occlusion by injection of particulate steroids. ${ }^{2}$

Intravascular injections of anesthetics can also result in cardiotoxicity. ${ }^{3}$ Kim et al. ${ }^{4}$ found that intravascular injections, including purely vascular and simultaneous perineural and vascular uptake, occurred at rates of $9.9 \%$ and $63.4 \%$ for lumbar and cervical transforaminal epidural injections, respectively.

Current methods for avoiding intravascular penetration are not always effective. Aspiration provides false negatives ${ }^{3,5}$; suction performed during this test can collapse a vessel and prevent backflow of blood. ${ }^{6}$ In a prospective study of transforaminal cervical epidural steroid injections, aspiration of blood into the hub of the needle was found to be $97 \%$ specific but only $45.9 \%$ sensitive for intravascular injection. ${ }^{7}$ The use of radiographic contrast injected under continuous fluoroscopy with or without digital subtraction is recommended to detect intravascular needle placement. ${ }^{2,8} \mathrm{~A}$ radio-opaque contrast injection may not always be effective, however; the spreading pattern can be fleeting and its interpretation is not always straightforward because an injection can be simultaneously intravascular and epidural. ${ }^{9}$ Furthermore, slight movements of the needle after the injection could result in a transition from an extravascular to an intravascular position.

Optical reflectance spectroscopy could potentially allow for a reliable detection of intravascular needle tip placement. With this technique, broadband light is delivered to the tissue, and it is subsequently scattered and absorbed. By spectrally resolving scattered light, the presence of specific absorbing molecules can be inferred. Optical absorption from oxyhemoglobin and deoxyhemoglobin is particularly prominent in the wavelength range of 500 to $600 \mathrm{~nm}^{10}$ Benaron et al. ${ }^{11,12}$ demonstrated that tissue oximetry can be performed with needle probes.

Recently, our research team introduced needles with optical fibers integrated into the cannula ${ }^{13}$ and into the stylet ${ }^{14}$ and an associated optical console ${ }^{15}$ to perform spectroscopic measurements during insertions to the epidural space and peripheral nerves. Pilot studies performed in swine, which involved insertions to the epidural space and the brachial plexus, provided initial indications that vascular penetration events can be detected with optical reflectance spectroscopy. ${ }^{16,17}$

The purpose of this study was to investigate whether optical reflectance spectroscopy has the potential to detect vascular penetration in humans. During blocks of the sympathetic chain and the communicating ramus in humans, a custom-designed needle 
stylet with integrated optical fibers was used to acquire reflectance spectra from tissues at the needle tip. The reflectance spectra were compared with the results of 2 current methods for detecting intravascular penetration, aspiration, and radio-opaque contrast injections.

\section{Methods}

\section{Patients}

The study was conducted at the Maastricht University Medical Centre (Maastricht, the Netherlands) with the Medical Ethics Committee approval (Medisch Ethische Commissie academisch ziekenhuis Maastricht, Maastricht, the Netherlands). A total of 10 patients, whose age ranged from 38 to 79 years, were included in the study. All patients provided written informed consent for their participation. Three of the patients were scheduled to receive lumbar sympathetic blocks for the treatment of complex regional pain syndrome or pain secondary to peripheral vascular disease. Seven patients were scheduled to receive a communicating ramus nerve block for the treatment or diagnosis of discogenic lower back pain. One insertion at the L4 level was performed for patients who received a lumbar sympathetic block, and 2 or 3 insertions at 2 levels around the corpus vertebrae of $L 4$ and $L 5$ were performed for those who received a communicating ramus block. In total, there were 18 insertions. All procedures were performed without sedation. Oxygen saturation and heart rate were monitored continuously, and an intravenous cannula was inserted for safety reasons.

\section{Optical stylet and console}

The custom optical stylet, developed by Philips Research (Eindhoven, the Netherlands) and InVivo GmbH (Schwerin, Germany), was compatible with a commercially available 20- gauge radiofrequency needle cannula (Cotop, Amsterdam, the Netherlands). The stylet was CE marked by In Vivo GmbH for peripheral nerve blocks in humans. The needle cannula had a length of $144 \mathrm{~mm}$ and a bevel angle of 20 degrees. Two optical fibers with core diameters of $100 \mathrm{Km}$ were integrated in the needle stylet. One fiber delivered broadband light to the tissue; the other received scattered light and directed it to the optical console. The optical console consisted of a tungsten-halogen light source and a spectrometer that resolved received light from the tissue across the wavelength range of 500 to $950 \mathrm{~nm}$. The optical console was previously described in detail. ${ }^{15}$ 


\section{Insertion protocol}

Fluoroscopic imaging with a mobile C-arm system (BV Endura; Philips Healthcare, Best, the Netherlands) was used for all procedures. The C-arm was first positioned anteroposteriorly to locate the appropriate vertebral level. The C-arm was then turned obliquely to determine the entry point, with a "tunnel" view. An intracutaneous injection of $1.0 \%$ lidocaine was performed with a 27-gauge needle for surface analgesia. Needle insertions toward the nerve targets with the radiofrequency needle and optical stylet were performed in accordance with standard practice. ${ }^{18}$

When the presumed nerve target region was reached, optical spectra and a fluoroscopic image were acquired. Five spectra were acquired; each acquisition was 0.5 seconds in duration. A background spectrum corresponding to each tissue spectrum was also acquired, with the broadband light shuttered to measure ambient light. Subsequently, the optical stylet was removed, and an aspiration test was performed. Radioopaque contrast (iohexol) was administered, and the pattern of contrast spread was noted. In case of intravascular detection, the needle was repositioned. When both tests for intravascular detection were negative and the characteristic spread of contrast around the nerve was noted, a diagnostic block or radiofrequency ablation was performed.

During the insertions, information about the optical spectra was unavailable to the practitioner to ensure that the trajectories of the needle tip were as similar as possible to those performed in normal clinical practice.

\section{Spectral analysis}

Spectral analysis was performed offline with custom software written in Matlab (MathWorks, Natick, Mass). Two pre-processing steps were performed, as detailed previously: subtraction of the background spectrum and an intensity calibration with a spectrum acquired from a white reflectance standard. ${ }^{15}$ Subsequently, a blood parameter, B, was calculated from each spectrum.

The blood parameter is a measure of the similarity of a spectrum acquired from the nerve target region, $S_{\text {target, }}$ to the spectrum acquired from an extracted blood sample, $\mathrm{S}_{\text {blood. }}$ This extracted blood sample was obtained from a volunteer who was distinct from the patients enrolled in the study. After hypodermic extraction from a superficial arm vein, the optical needle and stylet were positioned in this blood sample, such that the distance between the bevel surface and the walls of the surrounding vial was greater than $5 \mathrm{~mm}$. A total of 5 blood spectra and 5 background spectra were acquired within 1 minute of extraction, with exposure times of 0.5 seconds per spectrum. The blood parameter is given by:

$$
B=1 /\left|\left\langle S_{\text {target }}\right\rangle-S_{\text {blood }}\right|,
$$


where " \langle\rangle " denotes an average of the spectrum for 4 wavelengths: 529, 545, 570, and $584 \mathrm{~nm}$. At these wavelengths, optical absorption from hemoglobin is prominent so that there is a high contrast for a transition into a blood vessel, and the optical absorption coefficients of oxygenated and deoxygenated forms of hemoglobin are equal. The blood parameter is an empirical parameter that was developed as a relative measure to compare spectra; it does not provide a direct measure of the hemoglobin concentration. A high value for the blood parameter is indicative of a strong spectral similarity to blood.

To identify spectra that corresponded to vascular penetration events, a threshold for the blood parameter was calculated based on receiver operating characteristic analysis. Using exact logistic regression (with the "exlogistic" command in Stata 11, StataCorp LP, College Station, TX), the diagnostic odds ratio and corresponding confidence interval were calculated.

\section{Results}

During the 18 insertions performed during this study, 2 unintentional vascular penetration events were detected with aspiration and radio-opaque, contrast injection tests. During all other insertions, results from both of these 2 tests were negative. No complications were observed or reported.

In a fluoroscopic image acquired from the target location (sympathetic chain) during an insertion in which vascular penetration was not detected, contrast surrounding the nerve is clearly visible at the injection site (Figures 3A.1A, B). Optical absorption by hemoglobin was apparent in the optical spectra acquired from the target location. For wavelengths in the range of 500 to $600 \mathrm{~nm}$, where optical hemoglobin absorption is particularly high, intensities were lower than at higher wavelengths (600-900 nm).

In a fluoroscopic image acquired from an insertion in which vascular penetration was detected at the target location (sympathetic chain), contrast is not visible; its spread from the injection site was rapid (Figures 3A.1C, D). The effects of optical absorption by hemoglobin were more pronounced in the optical spectra acquired from this location than in those acquired from the aforementioned target location in which vascular penetration was not detected. Absorption by deoxyhemoglobin was not visually apparent in the spectra (the absorption spectrum of this form has a prominent peak centered at $757 \mathrm{~nm}$ that is absent in that of the oxygenated form ${ }^{10}$ ), which suggested that the punctured vessel was an artery.

Across all insertions, a high value of the blood parameter was associated with the detected vascular penetration at the nerve target region (Figure 3A.2). For both needle insertions in which vascular penetration was detected at the target location, the blood parameter was more than 4-fold higher than in the other 16 insertions. This difference 
in the blood parameter was statistically significant $(P=0.006)$, with a diagnostic odds ratio of 35.4 (confidence interval, 2.21 to $\infty$ ).
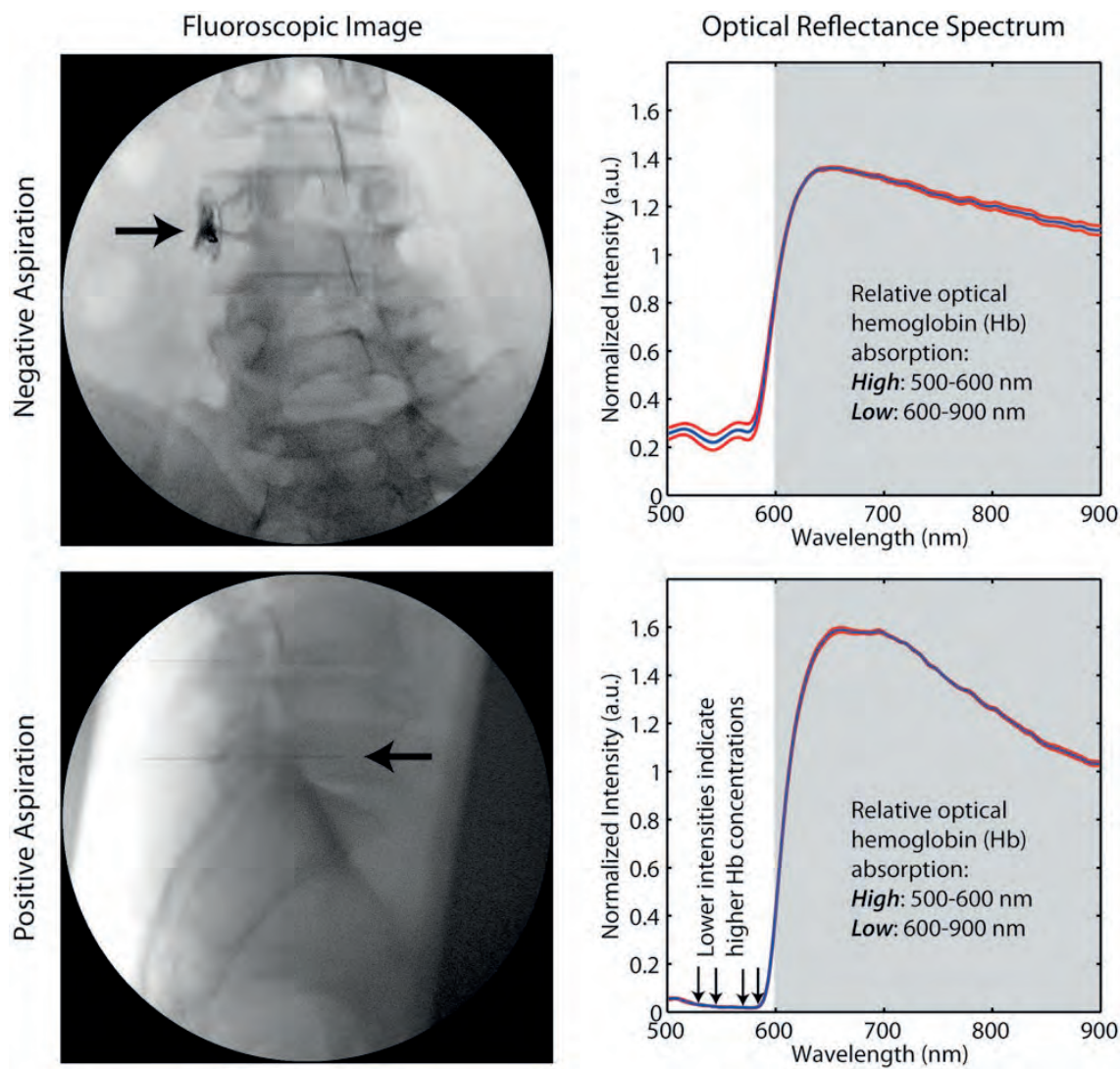

Figure 3A.1 Fluoroscopic images $(A, C)$ and optical spectra $(B, D)$ acquired from the nerve target regions during 2 insertions to the sympathetic chain (different subjects), in which results of the aspiration test for vascular access were negative $(A, B)$ and positive $(C, D)$. In the fluoroscopic images, the arrows indicate the location of the needle tip. Optical spectra corresponding to the 2 fluoroscopic images indicate the intensity of light received by the needle (linear units) as a function of the wavelength, normalized by the mean intensity. Mean spectral values (blue; middle) and SDs relative to the mean (red; top/bottom) are displayed as a function of wavelength. Specific wavelengths used to calculate the blood parameter (529, 545, 570, and $584 \mathrm{~nm}$ ) are indicated with arrows (D). At these wavelengths, optical absorption from hemoglobin is prominent so that there is high contrast for a transition into a blood vessel, and the optical absorption coefficients of oxygenated and deoxygenated forms of hemoglobin are equal. 


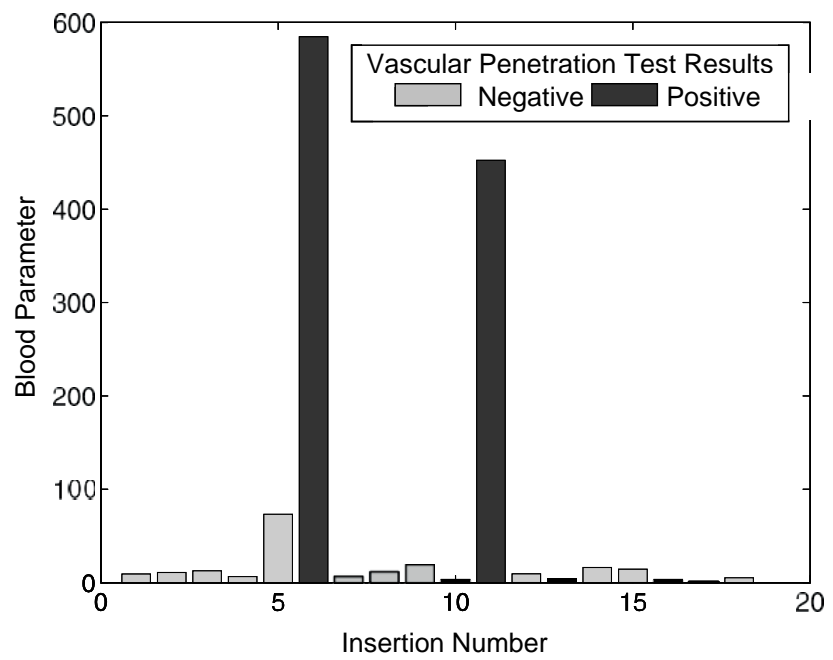

Figure 3A.2 Mean blood parameters calculated from the optical spectra that were acquired at the nerve target regions are shown. The blood parameter is a measure of the similarity of an optical spectrum to extracted blood (a higher value corresponds to greater similarity). The blood parameter was significantly higher when the aspiration test was positive (black) than when it was negative (gray).

\section{Discussion}

Fluoroscopic imaging can be invaluable for the guidance of nerve blocks, but it does not provide contrast for soft tissue structures like blood vessels. The optical stylet used in this study allowed for optical contrast to be derived from tissues at the needle tip without alterations to current needle insertion protocols. Optical absorption by hemoglobin as a means for generating contrast for blood that is complementary to traditional imaging modalities has shown promise in the context of near-infrared reflectance spectroscopy studies of brain injury in humans. ${ }^{19}$ To the authors' knowledge, this was the first study to demonstrate the use of reflectance spectroscopy to detect vascular penetration events during nerve blocks in humans.

For blocks of the lumbar sympathetic chain and the communicating ramus, intravascular injections and hematomas are rare ${ }^{20,21}$ but can lead to devastating complications. The optical stylet used in this study could, in principle, be modified to accommodate different needle cannula geometries; as such, it could potentially be used to detect intravascular needle placement across a broad range of interventional pain management and regional anesthesia procedures. Additional sources of contrast would be valuable to further reduce the risk of intravascular injections in transforaminal epidural steroid injections, where anatomic variation of the arteries is of concern (e.g., the artery 
of Adamkiewicz for lumbar and thoracic injections, ${ }^{22,23}$ and the vertebral and cervical arteries for cervical injections ${ }^{24}$ ).

This study had several limitations. The first relates to the method used to detect vascular penetration events. The criterion standard -detection with aspiration and with contrast injections- less than $100 \%$ sensitive. As a result, it is possible that vascular penetration without sequelae occurred in 1 or more of the insertions without being detected. This limitation could be overcome, in part, with computed tomography, but the gain in certainty would come at the expense of far greater doses of ionizing radiation, costs, and hospital staff time. The second limitation is that the relatively small sample size resulted in only 2 spectra that corresponded to vascular penetration events. Although the blood parameters obtained from these spectra were significantly larger than those of others, it is unclear to what extent vascular penetration could be detected in different contexts, including partial penetration of a vessel. In future studies, it would be interesting to investigate the extent to which vessel injury could be avoided. Even with the absence of intravascular injection, vessel injury can have fatal outcomes, as observed in 1 case with dissection of the vertebral artery with subsequent thrombosis ${ }^{25}$ and in a second case with a hematoma. ${ }^{20}$

The encouraging results of this study set the stage for larger scale studies, which could lead to algorithms that indicate when it is unsafe to perform an injection or administer radiofrequency ablation. Ultimately, analysis of the optical spectra might allow for chemometric analyses of heme proteins and other molecules that exhibit prominent optical absorption. With this information, arterial and venous blood could be differentiated, and tissue oxygenation could be estimated. Several factors would likely need to be taken into account, including the aggregation and flow of erythrocytes, the concentrations of plasma constituents such as bilirubin and platelets, and the effects of optical scattering. ${ }^{26}$ Aside from detecting vascular penetration events, chemometric analyses might allow for the positive detection of target nerves. The potential of this concept was recently demonstrated by 2 studies, in which the brachial plexus in swine was positively identified by optical spectroscopy performed with optical fibers integrated into the needle stylet. ${ }^{14,17}$

Detection of vascular penetration events with optical spectroscopy could also be valuable in the context of ultrasound-guided peripheral nerve block procedures. Ultrasound guidance is generally considered beneficial for reducing the risk of vascular penetration, although it is not clear whether this reduction is linked to a reduction in the risk of local anesthetic systemic toxicity. ${ }^{27}$ Visualizing vessels with ultrasound guidance can be challenging under certain conditions. For instance, acoustic shadowing can preclude visualization of vessels that are close to bones, and veins can be invisible when they are compressed. Pulsatile movements are not always apparent with small arteries, and there can be insufficient Doppler contrast for deep vessels due to signal attenuation. Intravascular injections have been reported even with the vascular and neural structures that were clearly visible with ultrasound. ${ }^{28}$ 
The results of this study provide an initial indication that vascular penetration events can be detected in humans with optical reflectance spectra acquired from tissues at the needle tip. Optical reflectance spectroscopy provides information that is complementary to fluoroscopy and current methods for detecting intravascular needle tip placement. 


\section{References}

1. Smuck M, Tang CT, Fuller BJ. Incidence of simultaneous epidural and vascular injection during cervical transforaminal epidural injections. Spine. 2009;34:E751-E755.

2. Rathmell JP, Aprill C, Bogduk N. Cervical transforaminal injection of steroids. Anesthesiology. 2004;100:1595-1600.

3. Petitjeans F, Mion G, Puidupin M, Tourtier JP, Hutson C, Saissy JM. Tachycardia and convulsions induced by accidental ropivicaine injection during sciatic block. Acta Anaesth Scand. 2002;46:616-617.

4. Kim DW, Han KR, Kim C, Chae YJ. Intravascular flow patterns in transforaminal epidural injections: a comparative study of the cervical and lumbar vertebral segments. Anesth Analg. 2009;109:233-239.

5. Ho KY. Vascular uptake of contrast despite negative aspiration in interlaminar cervical epidural injection. Pain Physician. 2006;9:267-268.

6. Checketts MR, Wildsmith JA. Accidental i.v. injection of local anesthetics: an avoidable event? $\mathrm{Br} \mathrm{J}$ Anaesth. 1998;80:710-711.

7. Furman MB, Giovanniello MT, O'Brien EM. Incidence of intravascular penetration in transforaminal cervical epidural steroid injections. Spine. 2003;28:21-25.

8. Jasper JF. Role of digital subtraction fluoroscopic imaging in detecting intravascular injections. Pain Physician. 2003;6:369-372.

9. Smuck M, Leung D. Inadvertent injection of a cervical radicular artery using an atraumatic pencil-point needle. Spine. 2011;36:E220-E223.

10. Schenkman KA, Marble DR, Feigl EO, Burns DH. Near-infrared spectroscopic measurement of myoglobin oxygen saturation in the presence of hemoglobin using partial least-squares analysis. Appl Spectrosc. 1999;53:325-331.

11. Benaron DA, Parachikov IH, Friedland S, et al. Continuous, noninvasive, and localized microvascular tissue oximetry using visible light spectroscopy. Anesthesiology. 2004;100:1469-1475.

12. Benaron DA, Parachikov IH, Cheong WF, et al. Design of a visible-light spectroscopy clinical tissue oximeter. J Biomed Opt. 2005;10:044005.

13. Desjardins $A E$, Hendriks BHW, van der Voort M, et al. Epidural needle with embedded optical fibers for spectroscopic differentiation of tissue: ex vivo feasibility study. Biomed Opt Express. 2011;2:1452-1461.

14. Desjardins AE, van der Voort M, Roggeveen $\mathrm{S}$, et al. Needle stylet with integrated optical fibers for spectroscopic contrast during peripheral nerve blocks. J Biomed Opt. 2011;16:077004.

15. Nachabe' R, Hendriks BHW, Desjardins AE, van der Voort M, van der Mark MB, Sterenborg HJCM. Estimation of lipid and water concentrations in scattering media with diffuse optical spectroscopy from 900 to $1600 \mathrm{~nm}$. J Biomed Opt. 2010;15:037015.

16. Rathmell JP, Desjardins AE, van der Voort M, et al. Identification of the epidural space with optical spectroscopy: an in vivo swine study. Anesthesiology. 2010;113:1406-1418.

17. Brynolf $M$, Sommer M, Desjardins $A E$, et al. Optical detection of the brachial plexus for peripheral nerve blocks: an in vivo swine study. Reg Anesth Pain Med. 2011;36:350-357.

18. Kallewaard JW, Terheggen MAMB, Groen GJ, et al. Discogenic low back pain. Pain Pract. 2007;10:560579.

19. Gopinath SP, Robertson CS, Contant CF, Narayan RK, Grossman RG, Chance R. Early detection of delayed traumatic intracranial hematomas using near-infrared spectroscopy. J Neurosurg. 1995;83:438-444.

20. Maier C, Gleim M, Weiss T, Stachetzki U, Nicolas V, Zenz M. Severe bleeding following lumbar sympathetic blockade in two patients under medication with irreversible platelet aggregation inhibitors. Anesthesiology. 2002;97:740-743.

21. Chandler G, Dalley G, Hemmer J Jr, Seely T. Gray ramus communicans nerve block: novel treatment approach for painful osteoporotic vertebral compression fracture. South Med J. 2001;94:387-393.

22. van Boxem K, Cheng J, Patijn J, et al. Lumbosacral radicular pain. Pain Practice. 2010;10:339-358.

23. Murthy NS, Maus TP, Behrns CL. Intraforaminal location of the great anterior radiculomedullary artery (artery of Adamkiewicz): a retrospective review. Pain Med. 2010;11:1756-1764. 


\section{Chapter 3A}

24. Huntoon MA. Anatomy of the cervical intervertebral foramina: vulnerable arteries and ischemic neurologic injuries after transforaminal epidural injections. Pain. 2005;117:104-111.

25. Rozin L, Rozin R, Koehler SA, et al. Death during transforaminal epidural steroid nerve root block (C7) due to perforation of the left vertebral artery. Am J Forensic Med Pathol. 2003;24:351-355.

26. Meinke M, Mueller G, Helfmann J, Friebel M. Optical properties of platelets and blood plasma and their influence on the optical behavior of whole blood in the visible to near infrared wavelength range. J Biomed Opt. 2007;12:014024.

27. Neal JM. Ultrasound-guided regional anesthesia and patient safety: an evidence-based analysis. Reg Anesth Pain Med. 2010;35:S59-S67.

28. Zetlaoui PJ, Labbe JP, Benhamou D. Ultrasound guidance for axillary plexus block does not prevent intravascular injection. Anesthesiology. 2008;108:761. 



\section{Chapter}

\section{Is it possible to identify intra- and extravascular needle placement with the STS system?}

Spectral tissue sensing to identify intra- and extravascular needle placement - a randomized single-blind controlled trial

Andrea J.R. Balthasar, Geert-Jan van Geffen, Marjolein van der Voort, Gerald W. Lucassen, Stefan Roggeveen, Ivar J. Bruaset, Joergen Bruhn PLoS One 2017;12(3):e0172662 


\section{Abstract}

Safe vascular access is a prerequisite for intravenous drug admission. Discrimination between intra- and extravascular needle position is essential for procedure safety. Spectral tissue sensing (STS), based on optical spectroscopy, can provide tissue information directly from the needle tip. The primary objective of the trial was to investigate if STS can reliably discriminate intra-vascular (venous) from non-vascular punctures. In 20 healthy volunteers, a needle with an STS stylet was inserted, and measurements were performed for two intended locations: the first was subcutaneous, while the second location was randomly selected as either subcutaneous or intravenous. The needle position was assessed using ultrasound (US) and aspiration. The operators who collected the data from the spectral device were blinded to the insertion and ultrasonographic visualization procedure and the physician was blinded to the spectral data. Following offline spectral analysis, a prediction of intravascular or subcutaneous needle placement was made and compared with the "true" needle tip position as indicated by US and aspiration. Data for 19 volunteers were included in the analysis. Six out of 8 intended vascular needle placements were defined as intravascular according to US and aspiration. The remaining two intended vascular needle placements were negative for aspiration. For the other 11 final needle locations, the needle was clearly subcutaneous according to US examination and no blood was aspirated. The Mann- Whitney U test yielded a $p$-value of 0.012 for the between-group comparison. The differences between extra- and intravascular were in the within-group comparison computed with the Wilcoxon signed-rank test was a p-value of 0.022. In conclusion, STS is a promising method for discriminating between intravascular and extravascular needle placement. The information provided by this method may complement current methods for detecting an intravascular needle position. 


\section{Introduction}

Safe vascular access is a prerequisite for intravenous drug admission. The opposite is true during locoregional anesthesia. In this case, local anesthetics should not be injected into a blood vessel but spread around the target nerve. Inadvertent vascular injections represent rare but serious complications. Several case reports have been published since the 1940s. ${ }^{1}$ The inadvertent injection of drugs into blood vessels can contribute to considerable morbidity ${ }^{2}$ and, therefore, precautions should be taken to reduce the risk of intravascular injection of local anesthetics. These precautions include aspiration before injection, administration of epidural test doses of local anesthetics, and incremental administration of small amounts of drugs. Imaging techniques such as ultrasonography (US), radiography or angiography are powerful tools to choose the correct injection point and may diminish, but do not exclude, the risk of inadvertent intravascular injections. ${ }^{3-6}$ In the case of intended venous injection, subcutaneous or inadvertent intra-arterial injections have to be avoided. ${ }^{7,8}$

Spectral tissue sensing (STS) is a new technique with the potential to provide information on the composition of the tissue in front of the needle tip. The technique is complementary to current imaging, aspiration and needling techniques and may be a useful tool to document and avoid inadvertent intravascular injection or to confirm an intravascular needle position. The optical method that forms the basis of the spectral tissue sensing technology is diffuse reflectance spectroscopy (DRS). The technique makes use of the phenomenon that if light is delivered to tissue, it is scattered and absorbed. The extent of scattering and absorption is determined by the composition of the tissue. For example, visible and near-infrared light are absorbed by hemoglobin, water, and lipids present in biological tissue. These substances show distinct absorption features. ${ }^{9-12}$ Well-established techniques that make use of this phenomenon are pulse oximetry and real-time gas analysis. ${ }^{13,14}$ The development of fiber-optic sensing technology has enabled DRS measurements directly via the tip of a needle. ${ }^{15-17}$ Needle stylets with DRS technology contained within standard needles allow for real-time spectral tissue sensing (STS). ${ }^{18}$ The STS concept used in this study has been tested in various other systems: in excised tissues, in vivo in pigs, and in vivo in humans., ${ }^{9,10,19}$ The theoretical advantage of STS compared to DSA and ultrasound is that STS provides real-time information on the tissue in front of the needle. Recently, Balthasar et al. demonstrated in a case series that STS was able to identify intravascular needle placement in $100 \%$ of cases compared to fluoroscopy and aspiration during invasive lumbar pain procedures. ${ }^{9}$ In that case report, intravascular needle placement was inadvertent and STS detected the intravascular placement in off-line analysis of the data after the procedures.

The goal of the current study was to investigate the potential of the STS method to discriminate intentional intravascular and non-vascular needle placement, by comparison with US and aspiration. 


\section{Methods}

\section{Study design}

The study was conducted at the Radboud University Nijmegen Medical Center in the period of 30th of June and 5th of July 2011. Since there was no follow up, the data collection was completed and the study ended on the 5th of July. After approval by the institutional review board (CMO Regio Arnhem-Nijmegen, Dr. F.Th.M. Huysmans, 29th of June 2011) and written informed consent, 20 healthy adult volunteers participated. Exclusion criteria were: pregnancy, photodynamic therapy, inability to give informed consent, category 2 and higher of the ASA physical status classification system.

The primary objective of the trial was to investigate if STS can reliably discriminate intravascular (venous) from non-vascular punctures.

Data were obtained at one time at two locations per volunteer. The first location in all 20 volunteers was subcutaneous needle placement, while second location was determined randomly. A computerized randomization scheme was used to determine needle placement for the second measurement either intravascularly $(n=10)$ or in subcutaneous tissue $(n=10)$. The operators who collected the data from the spectral device were blinded to the insertion and ultrasonographic visualization procedure and the physician was blinded to the spectral data. The obtained reflectance spectra were analyzed offline by an investigator blinded to the procedure. No follow up was planned (see also consort flow chart, Figure 3B.1 and consort checklist as supporting information).

According to the results of the spectral analysis, intravascular versus subcutaneous needle placement was predicted. The prediction by the STS method was compared with the "true" needle tip position as indicated by the aspiration of blood and an assessment by the physician during the procedure using out-of-plane (short-axis) ultrasound (US) guidance. A positive intravascular location was defined as a positive record made by the physician using US imaging in combination with aspiration of blood. We choose to combine both US imaging and aspiration, because aspiration alone has a sensitivity of only $25-47 \%$ to identify an intravascular needle position. ${ }^{21}$ Ultrasound is highly dependent on the experience and interpretation of the operator and can lead to false positive as well as negative results. ${ }^{5}$ 


\section{CONSORT 2010 Flow Diagram}

Enroliment

Assessed for eligibility $(n=20)$

Excluded $(n=0)$

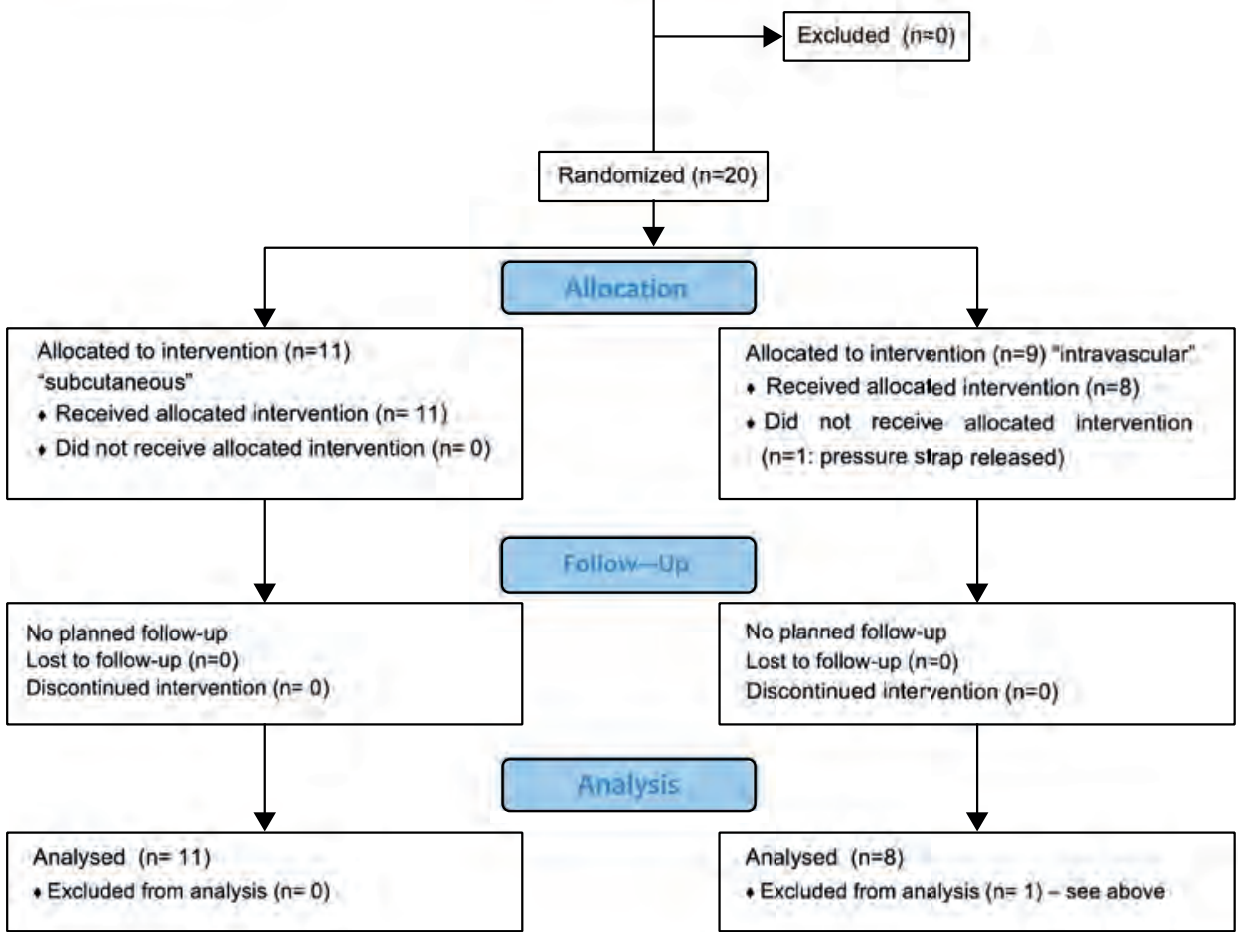

Figure 3B.1 Consort flowchart of the study.

\section{Study objective}

The objective of this observational study is to investigate if the optical tissue stylet technology can reliably discriminate intra-vascular (venous) from non-vascular punctures.

\section{Study registration}

This was very early phase research, sponsored by industry. Following the rules in the Netherlands for performing clinical research, the study was registered with the Dutch "Centrale Commissie Mensgebonden Onderzoek CCMO)" before enrolment was started, (see https://www.toetsingonline.nl/to/ccmo_search.nsf/ Searchform?OpenForm, 
search term "optical tissue stylet"Identifier: NL36528.091.11). The study was internationally registered after patient enrollment: Registry Url: http://www.isrctn.com/ ISRCTN15608308 Identifier: ISRCTN15608308.

\section{Device description}

The STS system consists of two parts: an STS stylet and an optical console (Figure 3B.2).

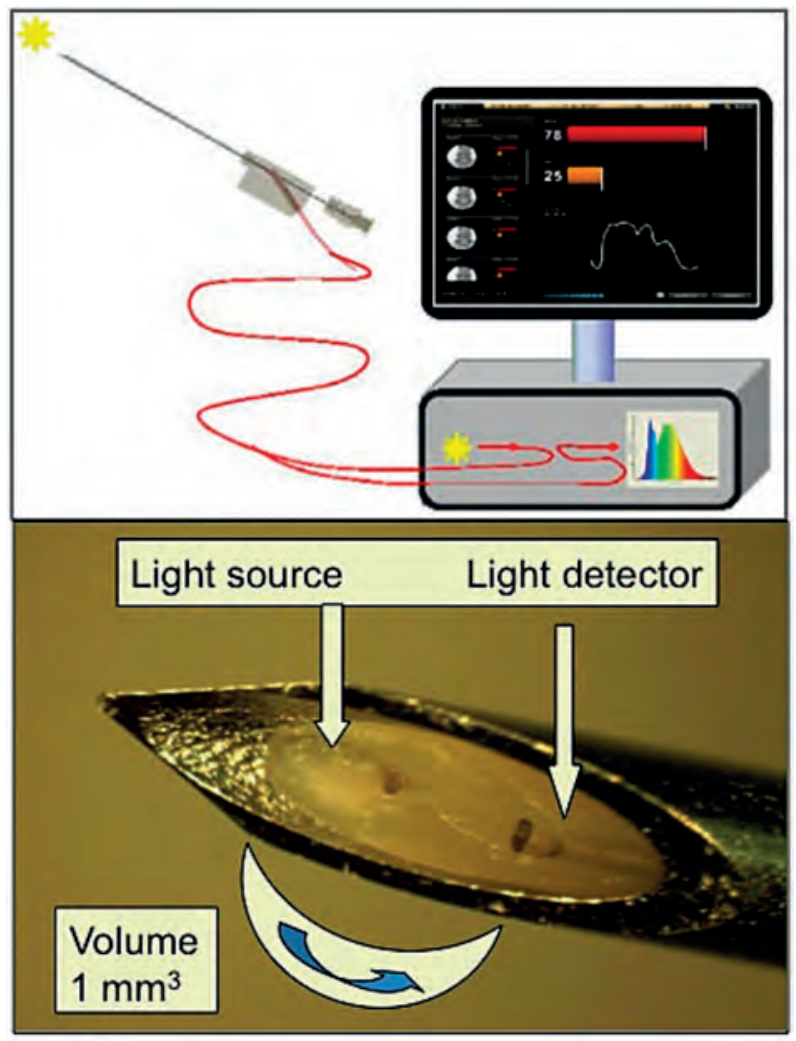

Figure 3B.2 Picture of the console and needle tip. Picture of the console (upper part) and needle tip with optical stylet (lower part). The drawing demonstrates the needle, which is connected to the console. The console is just a drawing and not an accurate image of the real one. The picture of the needle tip shows the two fibers and indicates the measuring volume.

The optical console contains a broadband (white) light source, two spectrometers and a computer board used to control the light source and data acquisition. The disposable sterile 20-G needles including the optical stylet are Conformite' Europe'enne (CE) marked (InVivo, Schwerin, Germany). The stylet contains two $100 \mu \mathrm{m}$ core fibers that are connected to a tungsten halogen broadband light source $(500-1600 \mathrm{~nm})$ and two 
spectrometers that receive the reflected light. A laptop computer is connected to control the light source, spectrometers and data acquisition. Details of the technical set up have been described elsewhere. ${ }^{9}$

\section{Study procedures}

After randomization, an experienced anesthesiologist performed all of the intravenous or subcutaneous needle insertions on the dorsal side of the hand or anterior forearm after local disinfection. During the entire procedure, a pressure strap was used on the forearm. Ultrasound imaging (SonoSite, MicroMaxs Turbo, linear transducer HFL 38 $\mathrm{mm}, 13-6 \mathrm{MHz}$ ) was used to guide all insertions, with the needle visualized out-of-plane (short axis). During needle insertion, spectral data were obtained twice for each volunteer: once subcutaneously and once at the intended location (subcutaneous or intravenous). Selection of the vein was done by the physician according to normal routine, preferably on the dorsal side of the hand. The needle was inserted with depth of at least $2 \mathrm{~mm}$. STS has a sensing volume of approximately $1 \mathrm{~mm}^{3}$. No light from the STS system was visible through the skin. US images were obtained at the final needle location. At the end of the procedure, the optical stylet was removed from the needle and aspiration was performed. To prevent dislocation of the needle tip during removal of the stylet, the tip was monitored continuously on the US screen during removal. As already pointed out, aspiration of blood in conjunction with a positive US image was used as a proof of an intravascular needle position. Absence of blood aspiration in conjunction with a negative US image was used to indicate a non-vascular needle position.

Only data that were collected during procedures according to this protocol (US images, aspiration and pressure strap during entire procedure) were included in the analysis.

\section{Spectral analysis}

Preprocessing of the spectral data (wavelength calibration, correction for instrument response and background signal, such as surrounding light) was performed in real time. After the clinical procedure, further analysis was performed using a custom-made program utilizing the software package Matlab (Mathworks, Natick, USA).

For each obtained spectrum, a "similarity" parameter was calculated, which indicates the similarity of the obtained spectrum, Starget, with a spectrum collected from a venous blood sample, Sblood. The blood similarity parameter is given by:

$$
B=1 / \mid<\text { Starget }>-<\text { Sblood }>\mid
$$

where " $<>$ " denotes the average of the spectral intensities at four wavelengths: 529 , 545,570 , and $584 \mathrm{~nm}$. A high value of the blood similarity parameter thus corresponds 
to high spectral similarity to blood. Because of the prominent spectral absorption from hemoglobin at these wavelengths, the blood similarity parameter provides high contrast for transition into a blood vessel. The approach followed here is similar to the analysis performed in an earlier study. ${ }^{9}$

\section{Sample size calculation}

We planned a study of a continuous response variable (namely the blood similarity parameter) from independent control and experimental subjects with one control per experimental subject. The sample size was determined based on the best available data at the time of the preparation of the study protocol, which were data on total hemoglobin estimates ( $\mathrm{HbT}$ ) from spectral fitting obtained in a previous study. ${ }^{11}$

In that study, 21 "not in blood vessel" spectral measurements resulted in mean and standard deviation values for $\mathrm{HbT}$ of $0.0292+/-0.0161$ and 10 "in blood vessel" spectral measurements resulted in mean and standard deviation values for $\mathrm{HbT}$ of $0.453 \mathrm{H}$ 0.156. These numbers were entered into a non-parametric Mann-Whitney test (unpaired t-test) using GraphPad StatMate 2.00 and GraphPad Instat software (www.graphpad.com/statmate/statmate.htm).

From this pilot data, it can be assumed that a detectable difference between the means of $\mathrm{HbT}$ of 0.115 is obtained for a sample size $n=10$ at a significance level of $\mathrm{P}=0.05$ and a power of 0.8 . Therefore, 10 subjects with one control per experimental subject and 10 control subjects were ample.

\section{Statistical analysis}

First, we computed blood similarity parameters for all volunteers and assessed whether a (range of) cut-off values can be determined to discriminate between values of extraand intravascular insertion.

Next, we compared blood similarity parameters between the two groups using only the second measurements (i.e. those at the intended location) and computed the Mann-Whitney $U$ test. We compared blood similarity parameters within the group of volunteers randomized to receive first an extravascular and then an intravascular insertion using the Wilcoxon signed-rank test, a nonparametric test for repeated measurements. A P-value of $\leq 0.05$ was considered to indicate statistical significance. All analyses were performed using $R$ version 3.1.3.

\section{Results}

Data from 19 of the 20 subjects were included in the analyses. One volunteer in the vascular group (volunteer 14) had to be excluded because data acquisition was not 
performed according to the protocol. Thus, 38 datasets were obtained in 19 volunteers, with two datasets per volunteer. The 38 sets comprised 19 subcutaneous sets from the first measurement location and 19 sets from the second measurement location, which were divided into 11 subcutaneous sets and 8 intravascular sets. In one case, although randomization asked for intravenous needle placement, but the physician performed an intentional subcutaneous placement.

In Figure 3B.3, typical spectra and the corresponding calculated blood similarity parameters for a subcutaneous needle tip position and an intravascular needle tip position are shown.
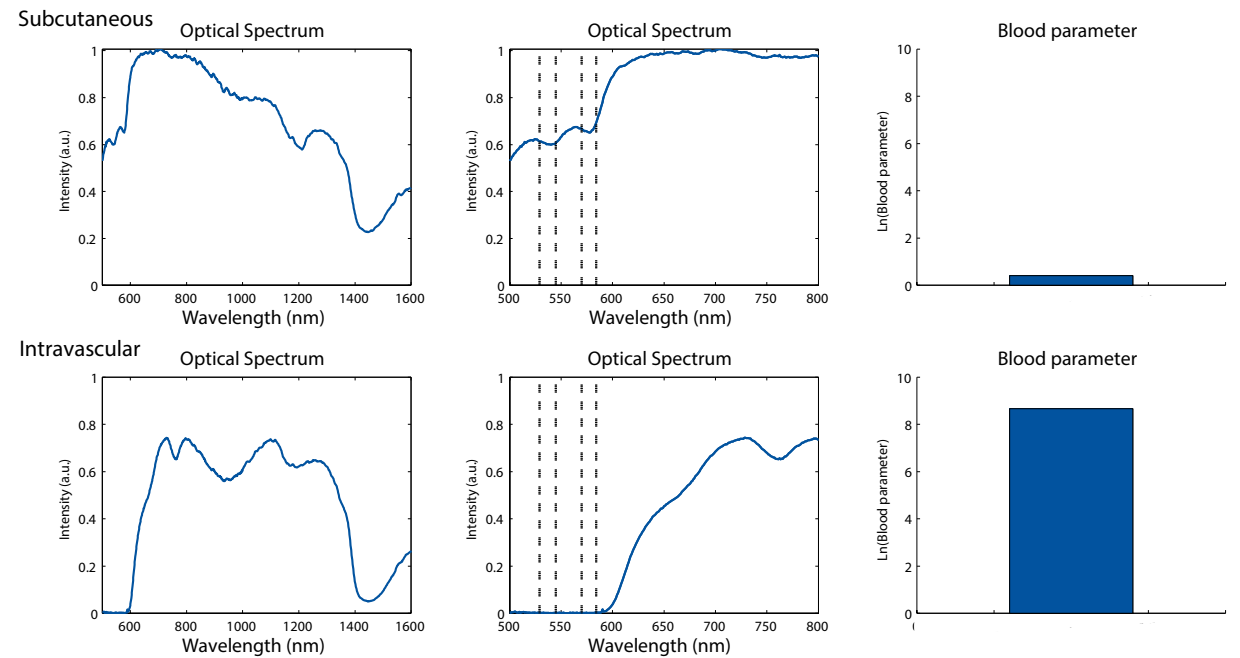

Figure 3B.3 Optical spectra and the corresponding blood similarity parameters. Spectra acquired from one volunteer for subcutaneous needle (upper panes) and intravascular needle positioning (lower panes), as confirmed by positive blood aspiration. Left: full spectra, indicating the intensity of light received by the stylet (linear arbitrary units, a.u.) as a function of the wavelength (nanometers, $\mathrm{nm}$ ). Center: an enlarged image of the spectrum that is used to determine the blood similarity parameter B (dashed lines indicate the wavelengths that are used for the calculation). Right: Ln (natural logarithm) of the blood similarity parameter as calculated for these two acquisitions.

Calculated blood similarity parameters for the spectra from 19 volunteers are given in Figure $3 b .4$, where the natural logarithm of the blood similarity parameter, $\operatorname{Ln}(B)$, is plotted. The natural logarithm is used here to capture the large dynamic range in the blood similarity parameter B. The similarity parameter is an empirical parameter that was developed as a relative measure to compare spectra. ${ }^{18}$ Table S3B.1 shows the measured blood similarity parameters of all patients. The statistical analyses were performed by an independent person (Sander van Kuijk, Clinical Epidemiologist, MUMC+). 


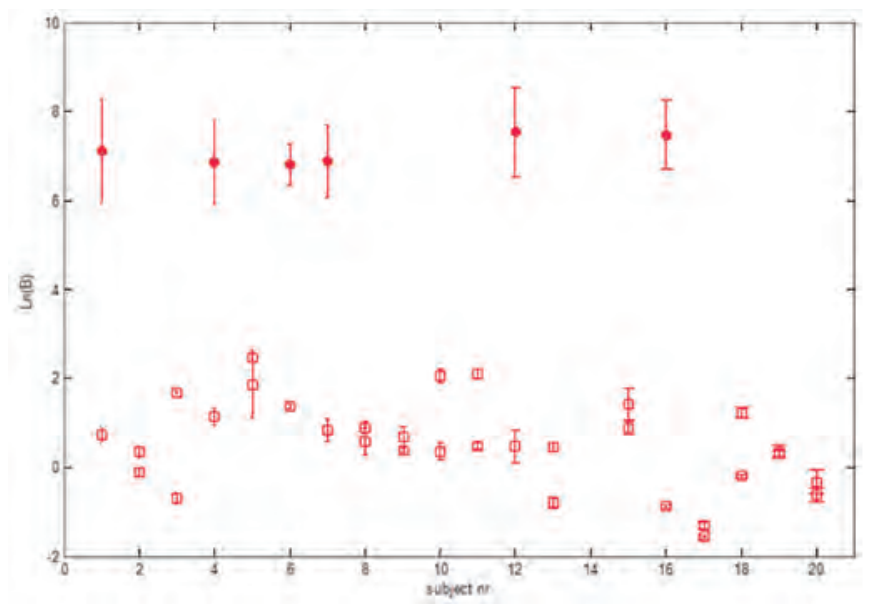

Figure 3B.4 All blood similarity parameters. Overview of all blood similarity parameters (B) determined for the different measurement locations in all volunteers. Results are plotted as the average natural logarithm Ln (B) (crosses), with standard deviations determined for the set of spectra acquired at each measurement location in each subject. Volunteer 14 was excluded. Because of the considerable differences in blood similarity parameters (B) between the two groups, more details in the data are visible by plotting $\operatorname{Ln}(B)$ instead of $B$ directly.

For 6 out of the 38 datasets, the blood similarity parameters were above the threshold, corresponding to a prediction of vascular puncture. All of these datasets corresponded with an intended vascular puncture, and were confirmed as vascular needle positions by US and the aspiration of blood. For 32 out of the 38 datasets, the blood similarity parameters were below the threshold and predicted a non-vascular needle placement. Of these 32 datasets, 19 were collected at the first, intended non-vascular measurement position. All of these were confirmed as non-vascular needle positions by US. Of the datasets where the blood similarity parameter predicted a non-vascular needle placement, 13 were collected at the second and final, measurement location. Of those 13, 11 datasets corresponded to an intended non-vascular needle position and two with an intended vascular position, confirmed by US but not by the aspiration of blood (inconclusive). Figure 3B.5 gives an overview of the study setup and of the results.

The agreement with the predictions based on STS measurements and confirmation by blood aspiration was $100 \%$. In comparison with US, predictions based on STS agreed with US for all intended non-vascular placements (100\%, negative control) and in six out of eight cases for intended vascular placements (agreement $75 \%$ ).

For the between-group comparison the natural logarithm of the mean of the extravascular and the intravascular groups was 1.36 and 8.42, respectively. The MannWhitney $U$ test yielded a P-value of 0.012. The differences between extra- and intravascular were even more pronounced in the within-group comparison in those individuals that received first an extravascular insertion, followed by an intravascular insertion. The 
log mean values were 0.56 and 8.42 for the extra- and intravascular measurements, respectively. The p-value computed with the Wilcoxon signed-rank test was 0.022 .

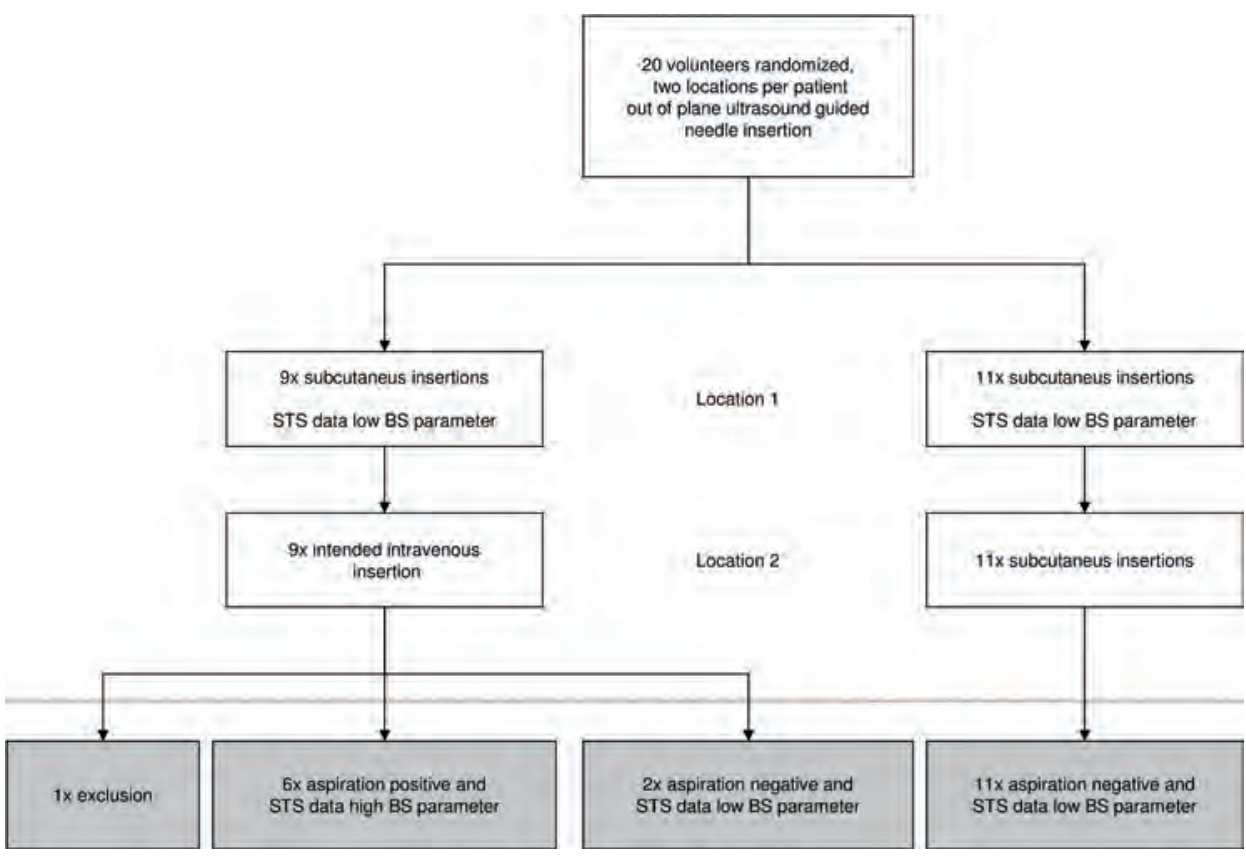

Figure 3B.5 Overview study design and results. Flow diagram of the study design including the results below the dotted line. STS: spectral tissue sensing, BS: blood similarity parameter.

\section{Discussion}

The results of the present study give some insight into the spectrum composition of intravascular versus extravascular needle placing. The ability to detect whether a needle or catheter is appropriately placed is of paramount importance for the prevention of inadvertent intravascular injection or for the confirmation of the correct, intravascular position. For instance, systemic toxicity due to inadvertent intravascular injection of local anesthetic occurs in 1 in 10000 patients after ultrasound guided axillary nerve block. $^{3}$

Balthasar et al. showed that vascular penetration events can be detected with spectral tissue sensing acquired from the tip of the needle. ${ }^{9}$ After assessing 18 needle insertions in 10 patients undergoing sympathetic chain or communicating ramus nerve block, two inadvertent vascular penetration events were detected. ${ }^{9}$ In the present study, we provide further evidence that an intravascular needle position can be detected by STS in human volunteers. 
The results of this study show that STS can detect intravascular needle placement in $100 \%$ of cases that were positive for the aspiration of blood. There was a correspondence of $75 \%$ between STS and US. In the other $25 \%$ of cases, the anesthesiologist assumed an intravenous needle position, based on the US image. In these cases, during the insertion procedure, the needle tip positions were judged as intravenous, but after removal of the stylet, there was no aspiration of blood. The spectra obtained for these three subjects suggested a subcutaneous needle position. Intravascular needle tip positions cannot be $100 \%$ excluded with this study design. Moreover, aspiration of blood may fail to detect intravascular needle or catheter positioning. To our knowledge, detailed data for the failure rate of aspiration in this particular setting (venous puncture with aspiration and a pressure strap) is unknown. Conversely, needle tip visualization using an out-of-plane (short axis) technique is challenging and the operator may have misjudged the image. ${ }^{22}$ It has been reported in the literature that for out-of-plane US guided procedures, the incidence of inadvertent posterior wall puncture is higher than for the in-plane approach. ${ }^{3,23}$ Nevertheless, there is no clear recommendation whether the out- or in-plane technique should be used during ultrasound guided vessel cannulation. ${ }^{24}$ From a practical point of view, out-of-plane visualization of the vessel is preferred. ${ }^{25}$ Hydrodissection is an alternative method that can be employed during US imaging to improve needle tip visualization and prevent accidental intravascular injection. $^{26}$

There are some limitations of the present study. We did not use additional in-plane imaging or the injection of saline. Practically, the stylet-containing needles used did not allow for the injection of fluid, and injection could have been performed only after removal of the stylet. In future studies, it will be important to employ disposables that allow for the injection and aspiration of fluids during the entire procedure.

Imaging techniques such as US or angiography are routinely used to confirm proper needle placement, for instance during central venous catheterization. ${ }^{27,28}$ An important caveat for the use of US guidance is that the needle and/or wire may not always be visualized in the vein. Confusion between the tip and shaft during US guidance of the needle can lead to inadvertent arterial cannulation. ${ }^{29}$ The occurrence of accidental arterial cannulation is usually recognizable from the color and the pulsatile nature of the blood back flow; however, case reports suggest that this is not always true. ${ }^{30}$ Pressure transducer monitoring and fluoroscopic guidance of the needle and catheter are considered to be reliable methods to place needles or catheters at the intended location. ${ }^{31}$ DSA, however, uses an iodinated contrast material, which may be contraindicated and requires exposure of the patient and physician to radiation. Without the regular use of DSA during pain procedures, serious adverse events have been reported. ${ }^{4}$

The advantages of STS are that it can provide information throughout the procedure, it does no harm to the patient or physician, it can be used to complement existing imaging modalities, STS information is less dependent on the skills and interpretation of the operator compared to DSA and ultrasound, and it does not disturb the workflow 
when integrated into the needle. The system used in this study was a preliminary version with a stylet. This stylet had to be removed before aspiration. It would be interesting in future studies to investigate the potential of a method to distinguish between venous and arterial blood during vascular procedures and compare with angiography and/or blood gas samples.

In conclusion, the results of this study demonstrate that STS is a promising method for discriminating between intravascular and extravascular needle placement. The information provided by this method may complement current methods for detecting and identifying an intravascular needle position and may improve patient safety. 


\section{References}

1. Cohen SM. Accidental intra-arterial injection of drugs. Lancet. 1948; 2(6524):409-17.

2. Sen S, Chini EN, Brown MJ. Complications after unintentional intra-arterial injection of drugs: risks, outcomes, and management strategies. Mayo Clin Proc. 2005; 80(6):783-95.

3. Zetlaoui PJ, Labbe JP, Benhamou D. Ultrasound guidance for axillary plexus block does not prevent intravascular injection. Anesthesiology. 2008; 108(4):761.

4. Chien GC, Candido KD. Digital Subtraction Angiography is Not the Answer for Safe Epidural Injections. Pain Physician. 2014; 17(3):E413-4.

5. Neal JM. Ultrasound-guided regional anesthesia and patient safety: An evidence-based analysis. Reg Anesth Pain Med. 2010; 35(2 Suppl):S59-67.

6. Hadzic A, Sala-Blanch X, Xu D. Ultrasound guidance may reduce but not eliminate complications of peripheral nerve blocks. Anesthesiology. 2008; 108(4):557-8.

7. Ball RD, Henao JP, Ibinson JW, Metro DG. Peripheral intravenous catheter infiltration: anesthesia providers do not adhere to their own ideas of best practice. J Clin Anesth. 2013; 25(2):115-20.

8. Patel RY, Friedman A, Shams JN, Silberzweig JE. Central venous catheter tip malposition. J Med Imaging Radiat Oncol. 2010; 54(1):35-42.

9. Balthasar A, Desjardins AE, van der Voort M, Lucassen GW, Roggeveen S, Wang K, et al. Optical detection of vascular penetration during nerve blocks: an in vivo human study. Reg Anesth Pain Med. 2012; 37(1):3-7.

10. Balthasar A, Desjardins AE, van der Voort M, Lucassen GW, Roggeveen S, Wang K, et al. Optical detection of peripheral nerves: an in vivo human study. Reg Anesth Pain Med. 2012; 37(3):277-82.

11. Jenkins JG. Some immediate serious complications of obstetric epidural analgesia and anaesthesia: a prospective study of 145,550 epidurals. Int J Obstet Anesth. 2005; 14(1):37-42.

12. Kenepp NB, Gutsche BB. Inadvertent intravascular injections during lumbar epidural anesthesia. Anesthesiology. 1981; 54(2):172-3.

13. Levin PD, Levin D, Avidan A. Medical aerosol propellant interference with infrared anaesthetic gas monitors. Br J Anaesth. 2004; 92(6):865-9.

14. Chan ED, Chan MM, Chan MM. Pulse oximetry: understanding its basic principles facilitates appreciation of its limitations. Respir Med. 2013; 107(6):789-99.

15. Bashkatov AN, Genina EA, Kochubey VI, and Tuchin VV. Optical properties of the subcutaneous adi- pose tissue in the spectral range 400-2500 nm. Optics and Spectroscopy. 2005; 99(5):836-42.

16. Bashkatov AN, Genina EA, Kochubey VI, and Tuchin VV. Optical properties of human skin, subcutane- ous and muscous tissues in the wavelength range from 400 t2000 nm. Journal of Physics D-Applied Physics. 2005; 38(15):2543-55.

17. Nachabe R, Hendriks BH, Desjardins AE, van der Voort M, van der Mark MB, Sterenborg HJ. Estima- tion of lipid and water concentrations in scattering media with diffuse optical spectroscopy from 900 to 1,600 nm. J Biomed Opt. 2010; 15(3):037015.

18. Desjardins AE, van der Voort M, Roggeveen S, Lucassen G, Bierhoff W, Hendriks BH, et al. Needle sty- let with integrated optical fibers for spectroscopic contrast during peripheral nerve blocks. J Biomed Opt. 2011; 16(7):077004.

19. Brynolf M, Sommer M, Desjardins AE, van der Voort M, Roggeveen S, Bierhoff W, et al. Optical detection of the brachial plexus for peripheral nerve blocks: an in vivo swine study. Reg Anesth Pain Med. 2011; 36(4):350-7.

20. Desjardins AE, Hendriks BH, van der Voort M, Nachabe R, Bierhoff W, Braun G, et al. Epidural needle with embedded optical fibers for spectroscopic differentiation of tissue: ex vivo feasibility study. Biomed Opt Express. 2011; 2(6):1452-61

21. Furman MB, Giovanniello MT, O’Brien EM. Incidence of intravascular penetration in transforaminal cervical epidural steroid injections. Spine. 2003; 28(1):21-5. 
22. Stone MB, Moon C, Sutijono D, Blaivas M. Needle tip visualization during ultrasound-guided vascular access: short-axis vs long-axis approach. Am J Emerg Med. 2010; 28(3):343-7.

23. Berk D, Gurkan Y, Kus A, Ulugol H, Solak M, Toker K. Ultrasound-guided radial arterial cannulation: long axis/in-plane versus short axis/out-of-plane approaches? J Clin Monit Comput. 2013; 27(3):319-24.

24. Blaivas M, Brannam L, Fernandez E. Short-axis versus long-axis approaches for teaching ultrasoundguided vascular access on a new inanimate model. Acad Emerg Med. 2003; 10(12):1307-11.

25. Lamperti M, Bodenham AR, Pittiruti M, Blaivas M, Augoustides JG, Elbarbary M, et al. International evidence-based recommendations on ultrasound-guided vascular access. Intens Care Med. 2012; 38 (7):1105-17.

26. Dufour E, Donat N, Jaziri S, Kurdi O, Couturier C, Dreyfus JF, et al. Ultrasound-guided perineural circumferential median nerve block with and without prior dextrose $5 \%$ hydrodissection: a prospective randomized double-blinded noninferiority trial. Anesth Analg. 2012; 115(3):728-33.

27. Jasper JF. Role of digital subtraction fluoroscopic imaging in detecting intravascular injections. Pain Physician. 2003; 6(3):369-72.

28. Wu SY, Ling $Q$, Cao LH, Wang J, Xu MX, Zeng WA. Real-time two-dimensional ultrasound guidance for central venous cannulation: a meta-analysis. Anesthesiology. 2013; 118(2):361-75.

29. Togashi K, Nandate K, Hoaglan C, Sherman B, Bowdle A. A multicenter evaluation of a compact, ster- ile, single-use pressure transducer for central venous catheter placement. Anesth Analg. 2013; 116(5): 1018-23.

30. Lim DY, Lee DW, Jang EA, Lee SH, Jeong HJ, Jeong CW, et al. Effects of inspired oxygen fraction in discriminating venous from arterial blood in percutaneous central venous catheterization under general anesthesia. Korean J Anesthesiol. 2012; 62(3):225-9.

31. Weiner MM, Geldard P, Mittnacht AJ. Ultrasound-guided vascular access: a comprehensive review. J Cardiothorac Vasc Anesth. 2013; 27(2):345-60. 


\section{Supplemental material}

Table S3B.1 Table containing blood similarity parameters (B) of all included individuals and measurements. Individual 14 was excluded. Number 1 means first position, number 2 second position. Intended subcutaneous position (S), intended vascular needle position (V). More data (ultrasound images, optical data and CRFs) could be found on figshare.com (project name: Spectral tissue sensing to identify intra-and extravascular needle placement).

\begin{tabular}{|c|c|c|c|}
\hline Volunteer & Location & Position & B \\
\hline 1 & 1 & $\mathrm{~S}$ & 2 \\
\hline 1 & 2 & V & 1501 \\
\hline 2 & 1 & $\mathrm{~S}$ & 1 \\
\hline 2 & 2 & $\mathrm{~S}$ & 1 \\
\hline 3 & 1 & $S$ & 0 \\
\hline 3 & 2 & S & 5 \\
\hline 4 & 1 & $\mathrm{~S}$ & 3 \\
\hline 4 & 2 & V & 28586 \\
\hline 5 & 1 & $S$ & 5 \\
\hline 5 & 2 & $\mathrm{~S}$ & 12 \\
\hline 6 & 1 & $\mathrm{~S}$ & 4 \\
\hline 6 & 2 & V & 1416 \\
\hline 7 & 1 & S & 2 \\
\hline 7 & 2 & V & 382 \\
\hline 8 & 1 & S & 2 \\
\hline 8 & 2 & $S$ & 2 \\
\hline 9 & 1 & $S$ & 2 \\
\hline 9 & 2 & $S$ & 1 \\
\hline 10 & 1 & S & 1 \\
\hline 10 & 2 & $S$ & 8 \\
\hline 11 & 1 & $S$ & 2 \\
\hline 11 & 2 & $S$ & 8 \\
\hline 12 & 1 & $S$ & 2 \\
\hline 12 & 2 & V & 1664 \\
\hline 13 & 1 & $S$ & 0 \\
\hline 13 & 2 & V & 2 \\
\hline 15 & 1 & $S$ & 2 \\
\hline 15 & 2 & $S$ & 4 \\
\hline 16 & 1 & $S$ & 0 \\
\hline 16 & 2 & V & 2915 \\
\hline 17 & 1 & $S$ & 0 \\
\hline 17 & 2 & $S$ & 0 \\
\hline 18 & 1 & S & 3 \\
\hline 18 & 2 & $S$ & 1 \\
\hline 19 & 1 & $S$ & 1 \\
\hline 19 & 2 & V & 1 \\
\hline 20 & 1 & $S$ & 1 \\
\hline 20 & 2 & $\mathrm{~S}$ & 1 \\
\hline
\end{tabular}






\section{Chapter \\ What is the optical signature of nerve tissue?}

Optical signature of nerve tissue - Explorative ex vivo study comparing optical, histological, and molecular characteristics of different adipose and nerve tissues

Andrea JR Balthasar, Torre M Bydlon, Hans Ippel, Marjolein van der Voort, Benno HW Hendriks, Gerald W Lucassen, Geert-Jan van Geffen, M van Kleef, Paul van Dijk, Arno Lataster Under review Journal of Lasers in Surgery and Medicine 


\section{Abstract}

\section{Background}

During several anesthesia procedures, needles are inserted through the skin of a patient to target nerves. In most cases, the needle traverses several tissues-skin, subcutaneous adipose tissue, muscles, nerves, and blood vessels-to reach the target nerve. A clear identification of the target nerve can improve the success of the nerve block and reduce the rate of complications. Patients and clinicians fear neurological complications, such as leg weakness or neuropathic pain, due to nerve damage. A clear identification of the tissue in front of the needle tip may further improve the performance and safety of needle-based interventions during anesthesia and pain therapy. Diffuse reflectance spectroscopy (DRS) can provide a quantitative measure of the tissue composition at the tip of a needle. The goal of the current study was to explore the nerve and adipose tissues encountered during needle insertion in more depth and to understand the morphological, biological, chemical, and optical characteristics that make up these tissues.

\section{Methods}

To compare characteristics of nerve tissue (sciatic nerve) and adipose tissues, the following techniques were used: histology, DRS, absorption spectrophotometry, high-resolution magic-angle spinning nuclear magnetic resonance (HR-MAS NMR) spectroscopy, and solution $2 \mathrm{D}^{13} \mathrm{C}^{-}{ }^{1} \mathrm{H}$ heteronuclear single-quantum coherence spectroscopy. Tissues from 5 human freshly frozen cadavers were examined.

\section{Results}

Histology clearly highlights a higher density of cellular nuclei, collagen, and cytoplasm in fascicular nerve tissue (IFAS). IFAS showed lower absorption of light around $1200 \mathrm{~nm}$ and $1750 \mathrm{~nm}$, higher absorption around $1500 \mathrm{~nm}$ and $2000 \mathrm{~nm}$, and a shift in the peak observed around $1000 \mathrm{~nm}$. DRS measurements showed a higher water percentage and collagen concentration in IFAS and a lower fat percentage compared to all other tissues. The scattering parameter (b) was highest in IFAS. The HR-MAS NMR data showed three extra chemical peak shifts in IFAS tissue.

\section{Conclusion}

Collagen, water, and cellular nuclei concentration are clearly different between nerve fascicular tissue and other adipose tissue and explain some of the differences observed in the optical absorption, DRS, and HR-NMR spectra of these tissues. Some differences observed between fascicular nerve tissue and adipose tissues can't yet be explained. 


\section{Introduction}

In anesthetic practice, the identification of different tissue types is essential. During several (regional) anesthesia and pain medicine procedures, needles are inserted through the skin of a patient to target nerves. Nerves are targeted to anesthetize an operation area, to treat acute postoperative pain, and to relieve chronic neuropathic pain. ${ }^{1}$ In most cases, the needle traverses several tissues-skin, subcutaneous adipose tissue, muscles, nerves, and blood vessels - to reach the target nerve. A clear identification of the target nerve can improve success of the nerve block and reduce the rate of complications. ${ }^{2}$ Current localization techniques, which include ultrasound or x-ray guidance, angiography, aspiration, and nerve stimulation, help the clinician to guide the needle to the target but are not without their limitations. ${ }^{2-7}$ Ultrasound with or without nerve stimulation is at the moment the most appropriate technique used during locoregional anesthesia. The identification of nerves with ultrasound is highly dependent on the anatomical knowledge, the skills and interpretation of the information by the operator, and the echogenic characteristics of the patient and the nerves. ${ }^{8,9}$ In locoregional anesthesia, nerve stimulation is only capable of identifying the nerve in $74.5 \%$ of the cases. ${ }^{10}$ Despite the use of ultrasound, nerve stimulation, and other precautions during injection, cases of nerve damage are still reported. ${ }^{11-13}$ The American Society of Regional Anesthesia and Pain Medicine states: "No nerve localization or monitoring technique has been shown to be clearly superior in terms of reducing the frequency of clinical injury" ${ }^{14}$ The incidence of complications after locoregional anesthesia is 4-6 per 10,000 blocks. ${ }^{15-18}$ Patients and clinicians fear neurological complications, such as leg weakness or neuropathic pain. ${ }^{19,20}$ Even temporary nerve damage has a major impact on the quality of life of the patient. ${ }^{21}$ Most neurological damage recovers in a year. However, more serious complications are reported after interventional pain therapy at the spinal level. Reports of spinal cord damage, brain infarctions, and death have been published. ${ }^{20,22-27}$

The majority of the complications is due to direct or indirect damage of the nerves and unintended injection of local anesthetics into blood vessels. ${ }^{19,28}$ Damage to more distal parts of a nerve tend to occur less often and are less severe than damage to more proximal parts. ${ }^{29-31}$ One explanation could be that peripheral nerves change their tissue composition from proximal to distal. The nerve root at the foraminal level of the spine is more solid and consists mostly of one large fascicle. ${ }^{32,33}$ Along their course, the fascicle branches and more connective and adipose tissue and blood vessels surround the fascicles in the nerve structure. ${ }^{29,30}$ The most vulnerable structures to needle damage are the nerve fascicles and the supplying microvessels. ${ }^{17,34}$

A clear identification of the tissue in front of the needle tip may further improve the performance and safety of needle-based interventions during anesthesia and pain therapy. One such technique that can provide a quantitative measure of the tissue composition (lipid, water, blood content, etc.) at the tip of a needle is diffuse reflectance spec- 
troscopy (DRS). ${ }^{35,36}$ The feasibility of DRS for a variety of clinical applications has already been reported. ${ }^{37-42}$ The basic principle involves delivering light into tissue with a small optical fiber and collecting reflected light with another optical fiber after it has undergone absorption and scattering events within the tissue. Depending on the presence and concentrations of light-absorbing molecules in the tissue, the light is absorbed differently, thus creating a specific fingerprint of the tissue. Prominent absorbers in biological tissue are hemoglobin, water, collagen, and lipids. ${ }^{35,36,43,44}$ Tissues that contain different amounts of these absorbers can be discriminated from each other. ${ }^{45}$ In an earlier in vivo study on optical detection of peripheral nerves, we concluded that differentiation between nerves in a fatty surrounding was difficult. ${ }^{41,42,46}$ In a more recent study in human cadavers, we found a sensitivity and specificity of $90 \%$ for discrimination of whole nerve tissue from surrounding tissues. ${ }^{33}$ In both of these studies, the nerve was considered as one entity, and we did not account for the various structures that make up the nerve bundle. All nerves were also treated equally.

The current study is meant to explore the nerve and adipose tissues encountered during needle insertion in more depth and to understand the morphological, biological, chemical, and optical characteristics that make up these tissues. A deeper understanding of these differences may help to improve the diagnostic capability of DRS for anesthesiology procedures.

\section{Methods}

The study was performed in collaboration with the Department of Anatomy and Embryology and the Department of Biochemistry of the Faculty of Health Medicine and Life Science (Maastricht University, Maastricht), the Department of Anesthesiology and Pain Medicine (University Hospital of Maastricht), and Philips Research (Eindhoven), all located in the Netherlands. A handwritten and signed codicil from each donor, as required by Dutch law for scientific research and education, is kept at the Department of Anatomy and Embryology.

\section{Sample size and statistical analysis}

To compare characteristics of human nerve tissue and adipose tissues, the following techniques were used: histology, DRS, absorption spectrophotometry, high-resolution magic-angle spinning nuclear magnetic resonance (HR-MAS NMR) spectroscopy, and solution $2 \mathrm{D}{ }^{13} \mathrm{C}-{ }^{1} \mathrm{H}$ heteronuclear single-quantum coherence (HSQC) spectroscopy. To our knowledge, no comparable studies have been reported. Due to the exploratory nature of the study, no input data for a power analysis were available. We therefore decided to only use descriptive statistics and indicate where possible confidence intervals and standard deviations. 


\section{Anatomy-histology}

\section{Anatomy}

Tissues from 5 human freshly frozen cadavers were examined. None of the individuals died due to a neurological disease. Table 4.1 provides an overview of the cadavers and the measurements performed on each sample. Certain measurement techniques were not available for all tissue samples.

Table 4.1 Overview of the cadavers included with patient characteristics and the measurements performed. Abbreviations: subcutaneous fat (SCF), fat close to sciatic nerve (adnexa / sliding fat, ADF), sciatic fascicular nerve (IFAS) tissue, sciatic non-fascicular nerve tissue (NFAS).

\begin{tabular}{|c|c|c|c|c|c|}
\hline \multirow{2}{*}{$\begin{array}{l}\text { Cadaver \# / Sex / } \\
\text { Age in years }\end{array}$} & \multirow{2}{*}{$\begin{array}{c}\text { Date tissue } \\
\text { extracted }\end{array}$} & \multirow{2}{*}{$\begin{array}{c}\text { DRS } \\
\text { measurements }\end{array}$} & \multirow{2}{*}{$\begin{array}{c}\text { Absorption } \\
\text { measurements }\end{array}$} & \multicolumn{2}{|c|}{ HNMR measurements } \\
\hline & & & & HR-MAS NMR / & $2 \mathrm{D}^{13} \mathrm{C}^{1} \mathrm{H} \mathrm{HSQC}$ \\
\hline 1/Female/76 & $\begin{array}{l}\text { November } \\
2014\end{array}$ & - & $\begin{array}{c}\text { SCF, ADF, NFAS, } \\
\text { IFAS }\end{array}$ & - & - \\
\hline 2/Male/78 & $\begin{array}{l}\text { November } \\
2014\end{array}$ & $\begin{array}{c}\text { SCF, ADF, NFAS, } \\
\text { IFAS }\end{array}$ & $\begin{array}{c}\text { SCF, ADF, NFAS, } \\
\text { IFAS }\end{array}$ & $\begin{array}{c}\text { SCF, ADF, NFAS, } \\
\text { IFAS }\end{array}$ & - \\
\hline 3/Male/86 & $\begin{array}{l}\text { November } \\
2014\end{array}$ & $\begin{array}{c}\text { SCF, ADF, NFAS, } \\
\text { IFAS }\end{array}$ & $\begin{array}{c}\text { SCF, ADF, NFAS, } \\
\text { IFAS }\end{array}$ & $\begin{array}{c}\text { SCF, ADF, NFAS, } \\
\text { IFAS }\end{array}$ & $\begin{array}{c}\text { SCF, ADF, NFAS, } \\
\text { IFAS }\end{array}$ \\
\hline 4/Female/91 & $\begin{array}{l}\text { August } \\
2015\end{array}$ & SCF, NFAS, IFAS & - & - & IFAS \\
\hline 5/Female/86 & $\begin{array}{c}\text { August } \\
2015\end{array}$ & SCF, NFAS, IFAS & - & - & - \\
\hline
\end{tabular}

As a target region, the sciatic nerve was chosen because of its size. In the upper leg, samples of subcutaneous fat (SCF), fat close to the sciatic nerve (adnexa / sliding fat, ADF), sciatic fascicular nerve (IFAS) tissue, and sciatic non-fascicular nerve (NFAS) tissue were dissected. Adipose tissue is composed of collagen and adipocytes filled with fat droplets; adipose tissue is referred to as fat in the text below. ${ }^{47}$ Figure 4.1 shows an anatomical drawing of the sciatic nerve, including descriptions of the different structures that make up the nerve bundle. The figure also shows a bifurcated sciatic nerve and the fascicles microscopically dissected from the nerve bundle.

Fat samples were collected macroscopically. Three SCF and ADF samples with a volume of $2 \mathrm{ml}$ per cadaver and a piece for cross-sectional histology were saved. For the collection of $2 \mathrm{ml}$ of fascicular and non-fascicular nerve tissue, a microscope was used. Immediately after preparation of the samples, samples were frozen (liquid nitrogen, -196 degrees Celsius and stored at -20 degrees Celsius) or fixed (4\% neutralbuffered formaldehyde for $24 \mathrm{~h}$ ). 


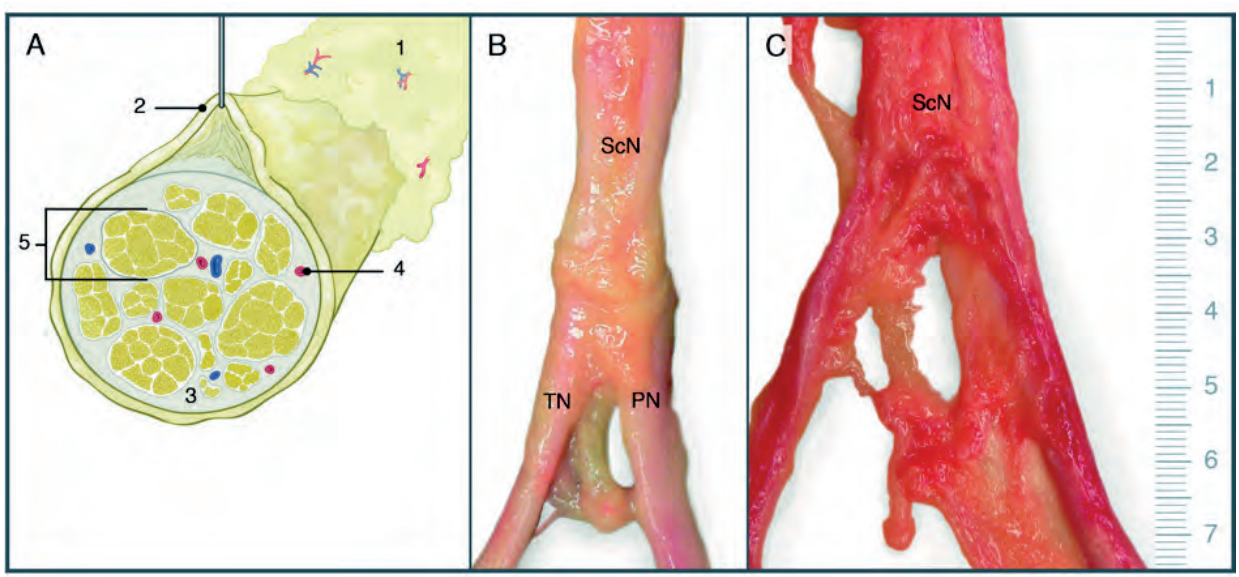

Figure 4.1 A: Anatomical drawing of a cross-section of a nerve: (1) subcutaneous adipose tissue, (2) adnex adipose tissue (sliding fat), (3) non-fascicular nerve tissue (NFAS) and epineurium, (4) blood vessel, and (5) fascicular nerve tissue (IFAS) and perineurium. B: Part of a sciatic nerve with bifurcation to the tibial (TN) and peroneal (PN) nerve. C: Fascicles of a sciatic nerve with most of the surrounding epineurium removed. Ruler $(\mathrm{mm}$ and $\mathrm{cm}$ ) belongs to pictures $\mathrm{B}$ and $\mathrm{C}$.

\section{Histology}

Three different staining techniques were used to differentiate between the different tissue compositions: Mayer's hematoxylin and eosin stain (H\&E stain) to color cell nuclei blue and cytoplasm pink ${ }^{48}$; osmium tetroxide $\left(\mathrm{OsO}_{4}\right)$ to color lipids black ${ }^{49}$; and MassonGoldner trichrome stain to visualize cell nuclei brown/black, cytoplasm stone red, collagenous fibers green, and erythrocytes orange. ${ }^{50}$ Parts of the samples containing fat were post-fixed in $1 \% \mathrm{OsO}_{4}$ in phosphate-buffered saline (PBS), $\mathrm{pH} 7.4$; the other samples were fixed in $4 \%$ neutral-buffered formaldehyde. After de-paraffinization, they were stained according to the selected staining protocol. ${ }^{51}$

The fixed and post-fixed tissues were embedded separately in paraffin. Then, 4- $\mu \mathrm{m}-$ thick histological sections of the sciatic nerve and IFAS tissue and 7- $\mu$ m-thick sections of the other tissues were cut on a Leica 2245 microtome. The sciatic nerve and IFAS tissues were cut transversely; the ADF, SCF, and NFAS tissues were cut in a direction suitable to obtain a large tissue area.

\section{Optical data collection}

\section{Absorption Spectrophotometry}

The tissues were removed from the freezer and brought to room temperature. For each type of tissue, a 1-mm cuvette was filled, while keeping the tissue homogenous throughout the length of the cuvette. 
A Perkin Elmer spectrophotometer was used to measure the total transmittance, collimated transmittance, and diffuse reflectance of each sample. The spectrophotometer was $0 \%$ and $100 \%$ background-corrected before any measurements. A stray light measurement was also taken to correct the diffuse reflectance spectra; this accounts for any light that might be scattered within the integrating sphere prior to reaching the sample itself. The measured reflectance $\left(R_{\text {meas }}\right)$ was corrected with known Spectralon data $\left(R_{100}\right)$ and the stray light $\left(R_{0}\right)$ measurement. Diffuse reflectance was therefore defined as:

$$
R=\frac{R_{100}\left(R_{\text {meas }}-R_{0}\right)}{100-R_{0}}
$$

Collimated transmittance $\left(T_{c}\right)$ and total transmittance $\left(T_{t}\right)$ measurements were used to calculate the diffuse transmittance $\left(T_{d}\right)$ :

$$
T_{d}=T_{t}-T_{c}
$$

The Kubelka-Munk equations ${ }^{52}$ were then used to calculate the absorption coefficient in units of $\mathrm{cm}^{-1}$.

\section{Diffuse reflectance spectroscopy measurements}

Frozen tissue samples were brought to room temperature and placed in glass cylinders. Ten diffuse reflectance spectra $(400-1600 \mathrm{~nm})$ were acquired from the tissue samples at 5 different locations with a custom-designed optical needle and an optical console. ${ }^{59}$ The optical needle had a $0.837-\mathrm{mm}$ distance between the two $200-\mu \mathrm{m}$ illumination and collection fibers and was used to relay light to and from the tissue to the optical console. The optical console consisted of a broadband light source and two spectrometers: one to record data in the visible wavelength range and the other for the near-infrared wavelengths. The spectra were corrected for daily variations in optical throughput using a Spectralon calibration standard. Several models have been described in the literature to express the intensity of light collected by a fiber after several scattering and absorption events in a diffuse medium. ${ }^{53-57}$ The model that is used in this paper is explained in earlier publications and corresponds to the solution of the diffusion equation for a semiinfinite medium and is a widely accepted model in the field of biomedical photonics. ${ }^{58-61}$ The fitting algorithm in the model utilizes the absorption coefficients of known tissue absorbers to determine the volume fraction of each absorbing element in the measured tissue, while taking into account tissue-scattering characteristics as well. For the analysis of the data collected for this study, the following absorbers were included in the fit model: oxygenated and deoxygenated hemoglobin, methemoglobin, water, fat (using the measured absorption coefficient of SCF), $\beta$-carotene, and collagen. Scattering- 
related parameters included were Mie scattering (Fmie), the Mie slope parameter (b), and the scattering at $800 \mathrm{~nm}(\mathrm{~S} 800)^{33}$

\section{HR- MAS and $2 \mathrm{D}^{13} \mathrm{C}^{1}{ }^{1} \mathrm{H}$ HSQC NMR data acquisition and processing}

${ }^{1} \mathrm{H}$ HR-MAS NMR (high-resolution magic-angle spinning nuclear magnetic resonance) spectra were acquired at $295 \mathrm{~K}$ on a Bruker Avance I narrow bore, $600 \mathrm{MHz}$ NMR spectrometer, equipped with a 14.1 Tesla superconducting magnet and a $4 \mathrm{~mm}{ }^{1} \mathrm{H} /{ }^{13} \mathrm{C} \mathrm{HR}$ MAS probe at a spinning rate of $6000 \mathrm{~Hz}$. The spectra were obtained under unlocked conditions. High-resolution NMR spectra of tissue extracts in deuterated chloroform $\left(\mathrm{CDCl}_{3}\right)$ and dimethyl sulfoxide (DMSO-d ${ }^{6}$ ) were recorded at $298 \mathrm{~K}$ on a Bruker Ascend HD $700 \mathrm{MHz}$ NMR spectrometer, equipped with a cryogenically cooled Triple Resonance Probe (TCl) probe. Typically, 32 to 128 scans were collected for a 1D proton spectrum using an inter-scan relaxation delay of 5 seconds.

At a later stage, a more detailed molecular analysis was done by acquiring a naturalabundance $2 \mathrm{D}^{13} \mathrm{C}-{ }^{1} \mathrm{H}$ HSQC spectrum of selected IFAS tissue extracts, in order to classify constitutive lipid components. Typically, 2 to 4 scans and 512 increments over a spectral width of ${ }^{13} \mathrm{C} 160 \mathrm{ppm}$ were collected.

\section{Results}

\section{Anatomy and Histology}

The H\&E-stained SCF and ADF slides showed large accumulations of fat cells separated by elongated and elaborated collagenous connective tissue structures and blood vessels (Figure 4.2 and 4.3).

The H\&E-stained cross-sections of the sciatic nerve showed several nerve fascicles. An outer, loose fibro-collagenous epineurium containing fat surrounded the intact nerve. The epineurium continued between the individual fascicle groups as an inner, inter-fascicular epineurium, with fat and blood vessels. The perineurium, consisting of concentric layers of flattened cells and collagenous connective tissue, surrounding and invading smaller groups of fascicles, and the endoneurium around and between groups of axons, were less visible. The osmium tetroxide (OsO4)-post-fixed cross-sections of the sciatic nerve showed the latter, more detailed aspects better, as well as the fat in the inter-fascicular epineurium and in the myelin sheaths around the myelinated axons (Figure 4.2). The cytoplasm surrounds the cell nuclei (stained blue in Figure 4.2A + C). In the fat cells, the cytoplasm is filled with (fused) fat vacuoles and forms a thin layer against the inner side of the cell membrane. 

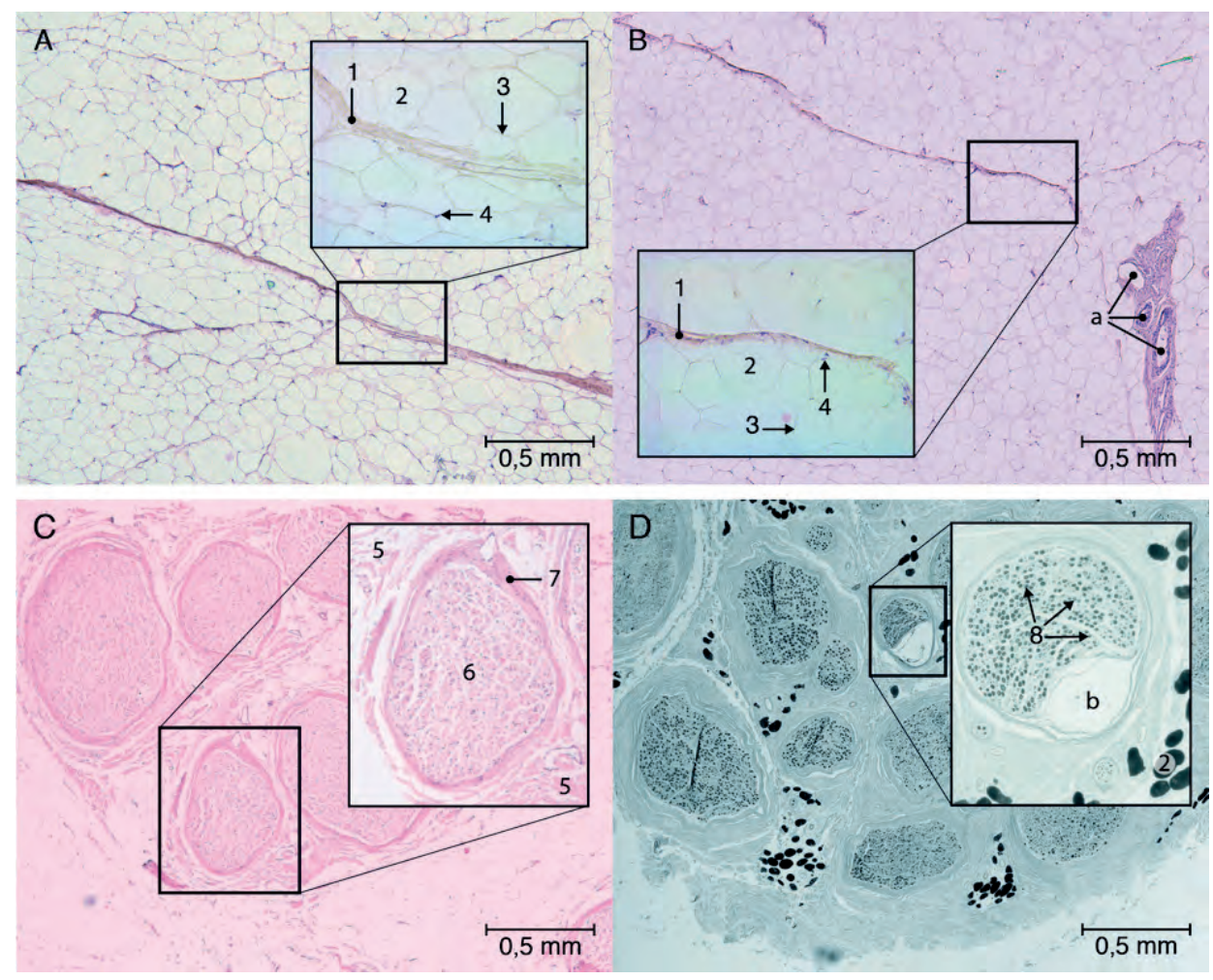

Figure 4.2 Histology of (A) SCF stained with H\&E, (B) ADF stained with H\&E, (C) cross-section of sciatic nerve stained with $H \& E$, and (D) cross-section of sciatic nerve stained with Osmium Tetroxide. H\&E colors cell nuclei blue and cytoplasm pink, and Osmium Tetroxide colors lipids black. (1) Collagenous fibers, (2) fat vacuole(s), (3) cytoplasm, (4) nucleus, (5) epineurium, (6) endoneurium, (7) perineurium, (8) myelin sheet (Schwann cells consisting of nuclei, cytoplasm, proteins, and lipids), (a) blood vessels, and (b) artifact.

In Figure 4.3, the SCF samples showed more collagenous fibers than the ADF samples. The IFAS sample in Figure 3 showed the same histology as the H\&E-stained crosssection in Figure 4.2. The H\&E- and Goldner's trichrome-stained NFAS sample showed less collagenous tissue and more adipose tissue than the IFAS.

Generally, the quality of the histology slides of Figure 4.3 was moderate because of the properties of the freshly frozen tissue. 

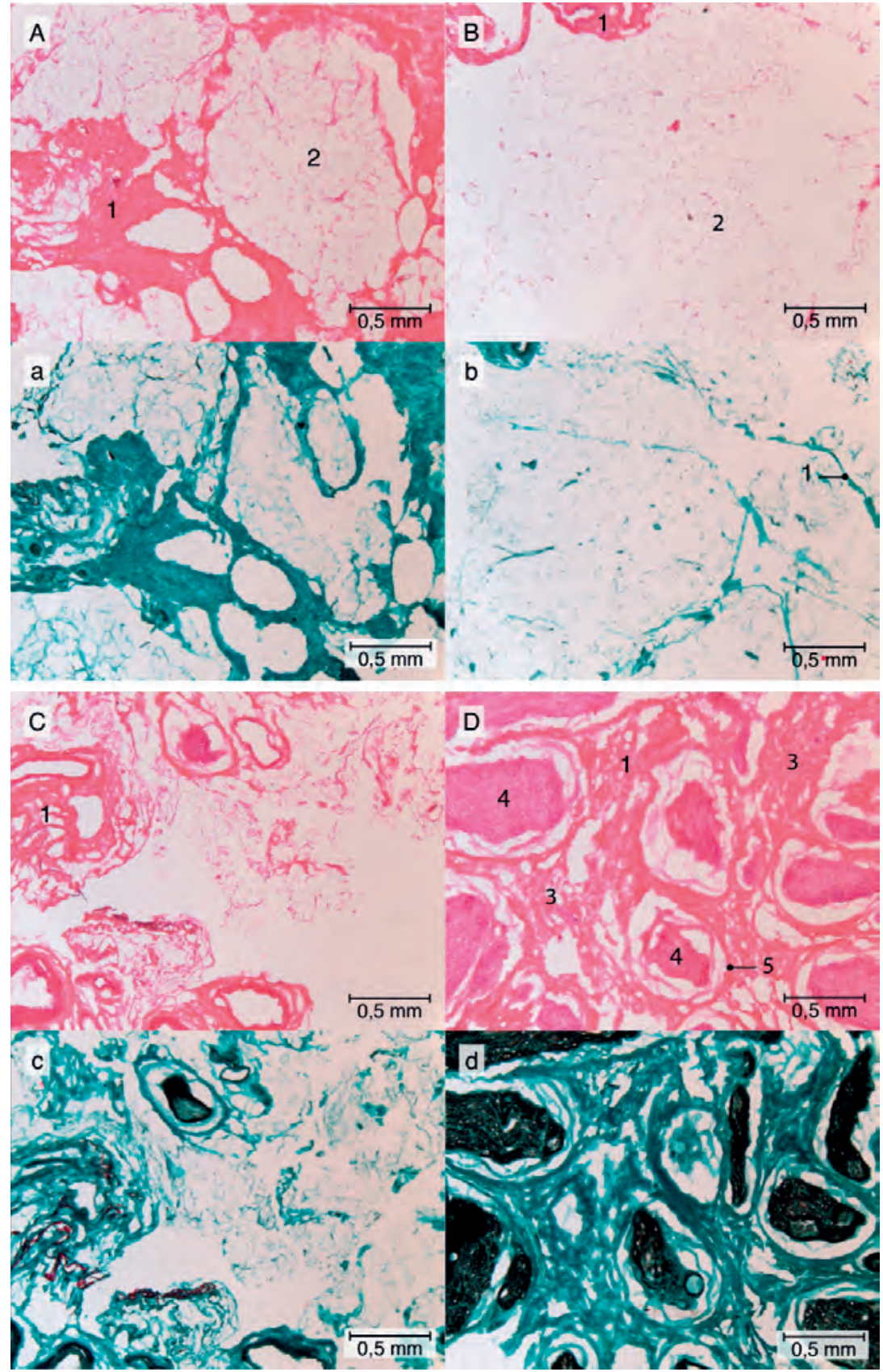

Figure 4.3 H\&E (red) and Masson-Goldner's trichrome staining (green/black). Masson-Goldner trichrome stain to visualize cell nuclei brown/black, cytoplasm stone red, collagenous fibers green, and erythrocytes orange. (A, a) Subcutaneous fat, $(B, b)$ adnex fat, $(C, C)$ non-fascicular nerve tissue, and (D, d) fascicular nerve tissue. (1) Collagenous fibers, (2) fat vacuole(s), (3) epineurium (containing also collagenous fibers), (4) endoneurium, and (5) perineurium. 


\section{Optical data}

\section{Absorption coefficients}

Figure 4.4 shows the measured absorption coefficients averaged across 3 cadavers. NFAS, SCF, and ADF show many similarities, while IFAS is most notably different around $1200 \mathrm{~nm}, 1500 \mathrm{~nm}, 1750 \mathrm{~nm}$, and $2000 \mathrm{~nm}$. Additionally, a red shift is observed in the peak near $1000 \mathrm{~nm}$, which is only seen in the IFAS tissues.

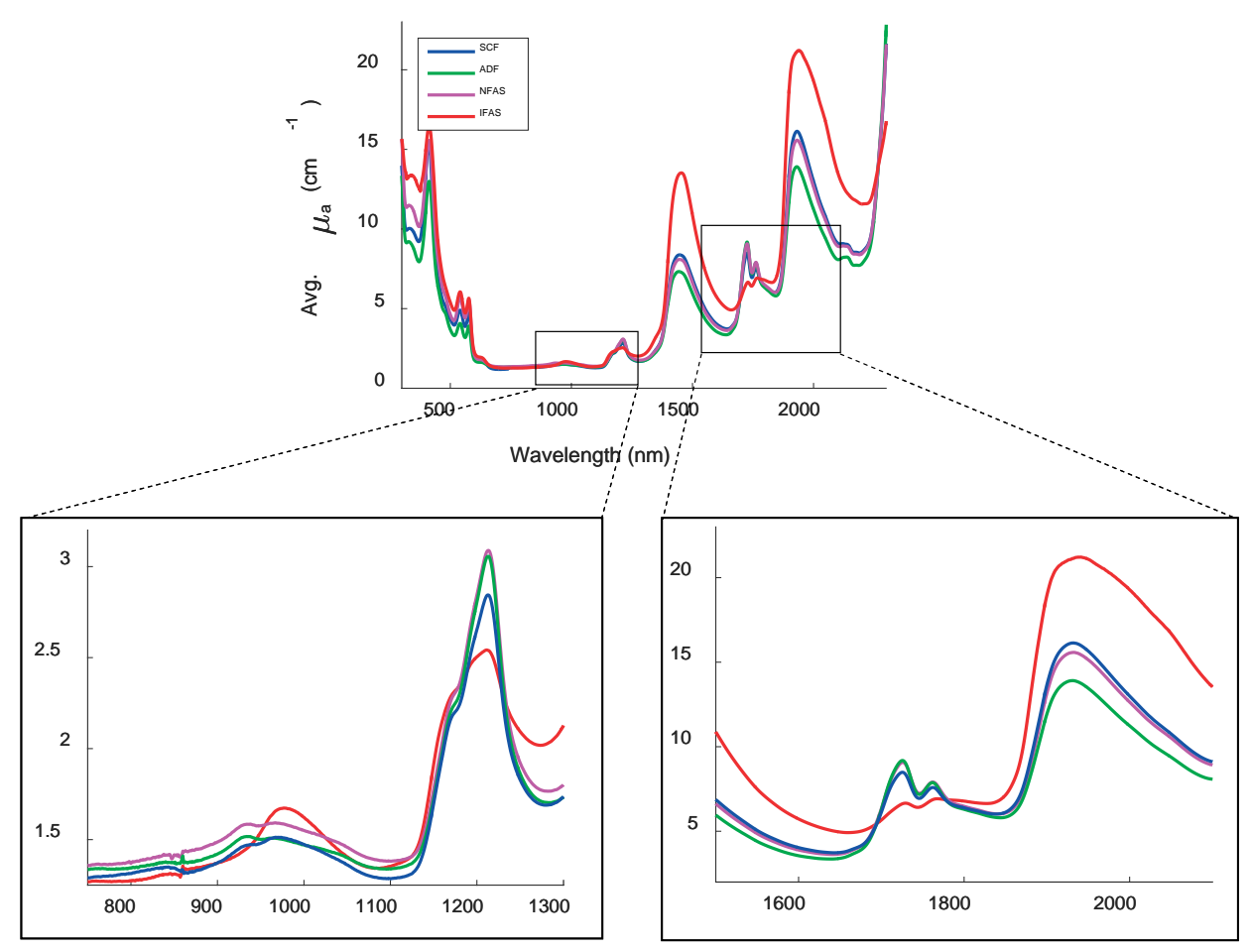

Figure 4.4 Tissue absorption coefficients measured with a spectrophotometer showing the full wavelength range and two separate wavelength regions.

\section{Diffuse reflectance spectra}

Figure 4.5A shows the DR spectra for the various measured tissues averaged over all measurement locations and 4 cadavers. Overall, little difference is seen between SCF and ADF. Although NFAS has a higher intensity, the general shape is similar to the ADF and SCF spectra. IFAS has the highest intensity, with differences seen in the slope (reflective of a scattering difference), around $1000 \mathrm{~nm}, 1200 \mathrm{~nm}$, and above $1400 \mathrm{~nm}$.

The fit parameters extracted from the spectra can be seen in Figures 5B-F. Blood and oxygen saturation (StO2) are highly variable between cadavers and do not show any 
trends. Collagen is higher in IFAS compared to NFAS, ADF, and SCF. The scattering parameters $\mathrm{S} 800$ and Fmie show little trending, while scattering slope (b) does appear higher in IFAS compared to the others. Fat is lowest in IFAS, with comparable values between the other tissues, while water shows the opposite trend. No trend is seen in $\beta$-carotene.

\section{HR-MAS and $2 \mathrm{D}^{13} \mathrm{C}-{ }^{1} \mathrm{H} H S Q C$ NMR spectroscopy}

Samples from cadavers 2 and 3 were studied by HR-MAS and $2 \mathrm{D}{ }^{13} \mathrm{C}-{ }^{1} \mathrm{H} H S Q C$ NMR spectroscopy. The proton HR-MAS NMR spectra of all four tissues (IFAS, NFAS, ADF, and SCF) are shown in Figure 4.6A and B. Eleven resolved proton signals are observed; corresponding chemical shifts and assigned chemical groups of the associated molecules are listed in Table 2. In order to compare the water content of the samples, the spectra were normalized on the intensities of the triglyceride- $\mathrm{CH}_{3}$ signal. Signals of triglyceride (signals 1, 2, 4-11) and water (signal 3) can be distinguished in the spectra of all samples. These were used to extract the water and lipid content of the different tissues, for comparison with the estimates of lipid and water content, based on DR measurements (Figure $6 \mathrm{C}$ and $\mathrm{D}$ ).

The IFAS spectrum (cadaver 3 ) contains a few additional signals (at 3.01, 3.20, and $5.77 \mathrm{ppm}$ ), which indicate the presence of a component comprised of unsaturated lipid protons (5.77 ppm) and a choline head group moiety (around $3 \mathrm{ppm}$ ). A search was done to identify the extra peaks. $2 \mathrm{D}{ }^{13} \mathrm{C}-{ }^{1} \mathrm{H}$ HSQC spectra were recorded to correlate protons to their bound carbon atoms. ${ }^{13} \mathrm{C}$ chemical shifts span a larger chemical shift range and are better indicators for the presence of certain chemical groups. Extraction of IFAS tissue material by $\mathrm{CDCl}_{3}-\mathrm{MeOH}(2: 1 \mathrm{v} / \mathrm{v})$ and/or DMSO-d ${ }^{6}$ was carried out to detect lipid constituents by means of $1 \mathrm{D} 1 \mathrm{H}$ and $2 \mathrm{D}{ }^{13} \mathrm{C}-{ }^{1} \mathrm{H} H S Q C$ solution NMR at 700 $\mathrm{MHz}$. These results confirm the triglyceride lipid composition from HR-MAS NMR but failed to detect the extra peaks of the unidentified compound (Supplements S4.1 and S4.2). DMSO extracts of pre-washed chloroform/methanol fractions of IFAS, where abundant apolar triglycerides are removed from solution, show indeed more polar lipid compounds in solution that contain resonances with chemical shifts compatible with the presence of choline groups (Supplement S4.1). The assignment of the main lipid signals is represented in Table 4.2. 

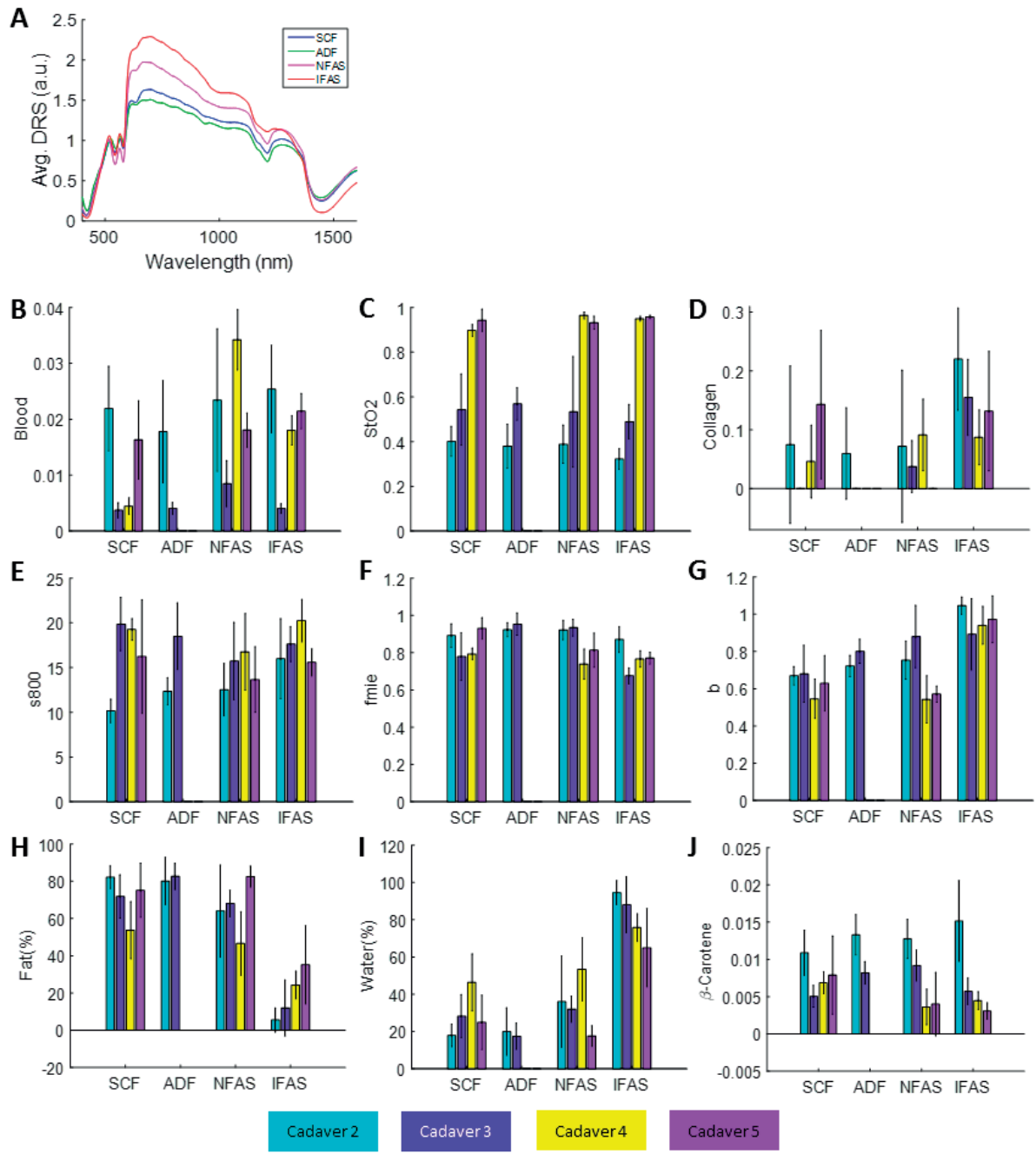

Figure 4.5 A) Diffuse reflectance spectra averaged over multiple sites and cadavers. B-J) Bar graphs of the fit parameters extracted from the diffuse reflectance spectra plotted per cadaver and per tissue type. The bar represents the average of all sites within a cadaver, the pink whisker represents the standard deviation, and the black whisker is the $95 \%$ confidence interval. 

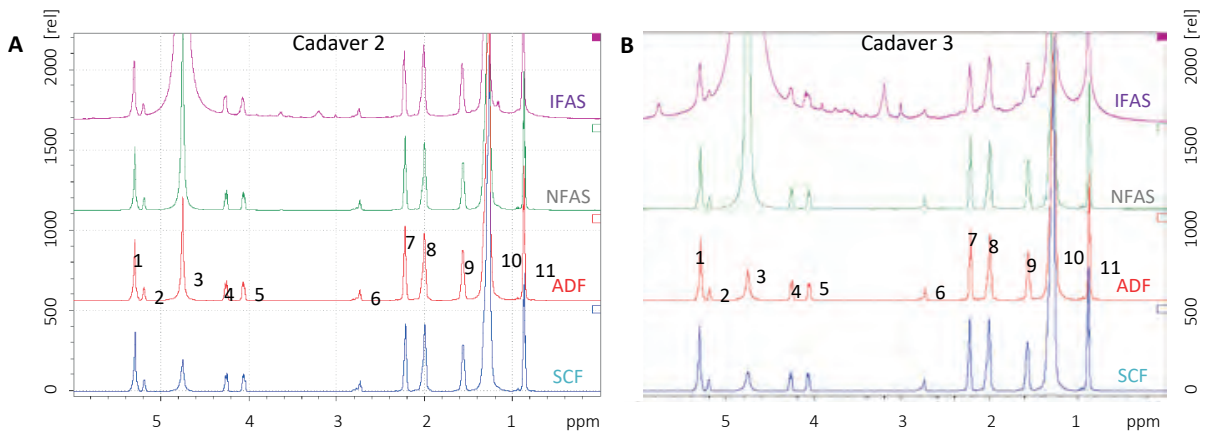

C

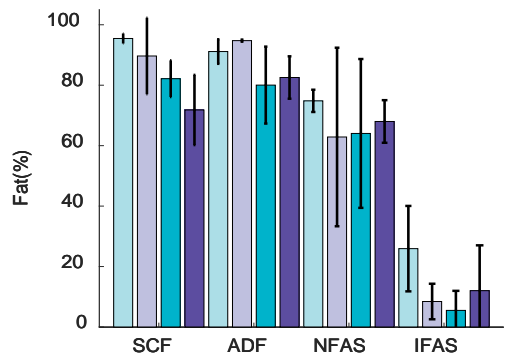

D

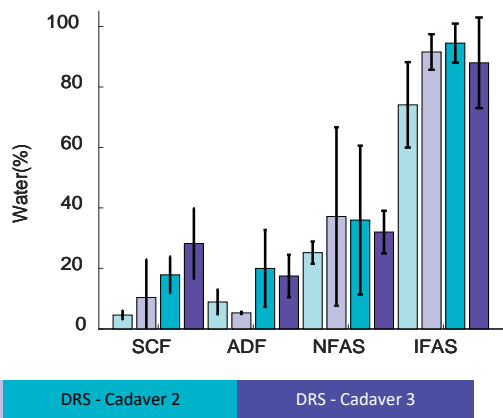

Figure 4.6 Results from cadavers 2 and 3. (A, B) HR-NMR results of IFAS, NFAS, ADF, and SCF (part per million (ppm), relative intensity (rel)). (C, D) Fat and water percentages extracted from the HRNMR data and DRS. Mean and 95\% confidence interval are presented.

Table 4.2 Assignment of the ${ }^{1} \mathrm{H}$-NMR signals. This table presents a detailed overview of the assignment of the 11 identified signals (Figure 4.6).

\begin{tabular}{|c|c|}
\hline Signal \# & Assignment \\
\hline 1 & $\mathrm{CH}$ of a lipid chain double-bond \\
\hline 2 & CHO of glycerol head group \\
\hline 3 & $\mathrm{H}_{2} \mathrm{O}$ \\
\hline 4 & $\mathrm{CH}_{2} \mathrm{O}$ of glycerol head group \\
\hline 5 & $\mathrm{CH}_{2} \mathrm{O}$ of glycerol head group \\
\hline 6 & $\mathrm{CH}_{2}$ in between two double-bonds \\
\hline 7 & $(\mathrm{C}=\mathrm{O}) \mathrm{CH}_{2}$ (next to ester) \\
\hline 8 & $\mathrm{CH}_{2}$ next to double-bond \\
\hline 9 & $(\mathrm{C}=\mathrm{O}) \mathrm{CH}_{2} \mathrm{CH}_{2}$ \\
\hline 10 & $\mathrm{CH}_{2}$ \\
\hline 11 & $\mathrm{CH}_{3}$ \\
\hline
\end{tabular}




\section{Discussion}

Discrimination of nerve fascicles from other surrounding tissues is essential to prevent possible complications due to percutaneous needle-based interventional procedures in anestheticpractice. Previous studies have shown that DRS can differentiate between adipose tissue, muscle, and blood. ${ }^{33,39-42,46}$ Our previous ex vivo DRS study demonstrated a sensitivity and specificity of $90 \%$ in discriminating whole nerve tissue compared to surrounding tissue. ${ }^{33}$ The aim of the current study was to investigate the basis for the difference between the DRS data of IFAS and surrounding tissues, by using histology, optical absorption measurements, DRS, and H-NMR and comparing the results.

Table 4.3 provides an overview of the main differences between IFAS and the other tissue, as observed with the various measurement techniques. Here, we summarize the findings:

Histology clearly highlights a higher density of cellular nuclei, collagen, and cytoplasm (consisting for $>80 \%$ out of water) in IFAS tissue (Figures 4.2 and 4.3 ).

Optical absorption measurements also showed clear differences between IFAS and the other tissues: compared to the other tissues, IFAS showed lower absorption around $1200 \mathrm{~nm}$ and $1750 \mathrm{~nm}$, higher absorption around $1500 \mathrm{~nm}$ and $2000 \mathrm{~nm}$, and a shift in the peak observed around $1000 \mathrm{~nm}$.

The estimates of chromophore concentrations and scattering parameters based on the DRS measurements showed a higher water percentage in IFAS and a lower fat percentage compared to all other tissues. Also, the collagen concentration appeared higher in IFAS compared to ADF and SCF. The scattering parameter (b) related to Mie scattering was highest in IFAS.

Finally, the HR-MAS NMR data showed three extra chemical peak shifts in IFAS tissue (3.0-3.2, 3.4, and 5.7-5.8 ppm). Estimates of the water and lipid content based on the HR-MAS NMR data indicated a high water and low lipid content in IFAS and vice versa for the other tissues.

When comparing the observations from the different methods, consistencies are observed. Estimates of the water and lipid contents appear consistent for DRS and HRNMR and are confirmed by histology. Also, the differences in optical absorption can probably be explained by this: the shape difference around $1200 \mathrm{~nm}$ is likely due to a lack of fatty tissue in the IFAS sample.

The collagen content estimated from DRS is highest in IFAS, which is confirmed by histology, as well. Then, looking again at the optical absorption, the lower absorption in IFAS $>1400 \mathrm{~nm}$ is likely caused by increased absorption by water and collagen.

Scattering is related to refractive index changes present in the tissue-i.e., to the inhomogeneity of the tissue. The high scattering parameter (b) observed by DRS in IFAS may therefore be related to larger inhomogeneity in the distribution of the collagen content and higher density of cellular nuclei, as seen in histology. 
Some observations from one method could clearly be related to findings from the other methods: the 1000-nm dip present most prominently in the DRS spectra of IFAS occurs at the same wavelength as the shifted optical absorption curve. This could be an $\mathrm{OH}$ second overtone, which likely comes from water. The fact that this shift is observed in IFAS could be consistent with the higher water content in that tissue. However, the shift may be attributed to some other substance, like special myelin proteins only found in IFAS tissue, ${ }^{62,63}$ as well. Other observations not easily explained are the increased optical absorption between 1600 and $2300 \mathrm{~nm}$; this may be related to a CH combination band. ${ }^{64}$ Also, the additional peaks observed in HR-NMR could not be directly related to the other measurements.

No significant inconsistencies between the findings from the different methods were observed.

Table 4.3 Overview of the differences in histology, optical, and HR-MAS NMR data between SCF, ADF, NFAS, and IFAS.

\begin{tabular}{ll}
\hline Measurement & IFAS vs. NFAS, ADF, SCF \\
& $\uparrow$ Density of cell nuclei (HE staining) \\
& $\uparrow$ Myelin (OsO $\left.{ }_{4}\right)$ \\
& $\uparrow$ Collagen (Goldner's Trichrome) \\
& $\uparrow$ Cytoplasm (water) \\
Histology & $\downarrow$ Fat concentration \\
& $\downarrow 1200$ and $1750 \mathrm{~nm}$ \\
& $\uparrow 1300-1600,1900-2200 \mathrm{~nm}$ \\
Absorption & Peak shift 900 and $1000 \mathrm{~nm}$ \\
\hline & $\uparrow$ H2O, collagen concentration \\
DRS & $\downarrow$ Fat concentration \\
& $\uparrow$ Scattering parameter (b) \\
\hline HR-NMR MAS & Extra chemical peak shifts 3.0-3.2, 3.4, 5.7- 5.8 ppm \\
\hline
\end{tabular}

Additional studies were performed to find an explanation for the additional peaks in the HR-NMR measurements. We suspected sphingomyelin to be the cause of these additional peaks, because it is a lipid found mainly in cell membranes of peripheral nerves, especially in the membranous myelin sheath that surrounds nerve cell axons, ${ }^{63,65}$ Additionally, the myelin sheath of a peripheral nerve consists of typical proteins, like $P_{0}$ (>50\% of the proteins), $P_{1}$, protein- 22 , and $40 \%$ of water. ${ }^{63}$

HR-MAS NMR was performed on pure cholesterol and sphingomyelin from chicken egg yolk (Sigma Aldrich, Product Number S0756, white powder, >95\% purity). Optical absorption and DRS measurements were performed in the sphingomyelin material. However, the results of those measurements failed to explain the additional HR-MAS NMR peaks in IFAS: the extra peaks in the HR-MAS NMR spectra do not match with the spectrum of pure cholesterol or sphingomyelin. Also, the DRS and absorption measurements of sphingomyelin did not show the same shifts and peaks as IFAS. 
The extra search for molecular identification of the extra peak shifts by $2 \mathrm{D}{ }^{13} \mathrm{C}-{ }^{1} \mathrm{H}$ HSQC confirmed the triglyceride lipid composition from HR-MAS NMR but failed to clearly explain the extra peaks of the unidentified compound (Supplements S4.1 and S4.2).

One possible explanation why we were not able to finally clarify the role of sphingomyelin is that sphingomyelin is strongly bound to the protein matrix inside nerve tissue and is therefore difficult to extract and dissolve. ${ }^{63}$ We further note that ex vivo human protein-bound sphingomyelin and the sphingomyelin of chicken yolk are molecularly different; so, the used material might not have been the best to test the hypothesis. However, no alternative materials were readily available.

Comparing these results with the results of Hendriks et al., the differences in collagen, water, scattering, and fat were similar. This study did not find differences in $\beta$ carotene. ${ }^{33}$ This may be due to individual differences between the cadavers, such as diet and percentage of subcutaneous fat, which influences $\beta$-carotene percentage.

This study expanded the optical database of the DRS of different human tissues. Optical data of blood, muscle, and fat are already well described. ${ }^{37-42,59,60}$ The former in vivo studies used a spectrum from 500-1600 nm. This study demonstrates that it could be interesting to use a wider range to discriminate IFAS. A study performed by Schols et al. used DRS up to $1830 \mathrm{~nm}$ in an in vivo setting to differentiate adipose from nerve tissue. $^{66}$ Stelzle et al. reported optical nerve detection by DRS in pigs (ex vivo). They could differentiate between nerve tissue derived from bone and from salivary glands with a sensitivity of approximately $83 \%$ and a specificity of $78 \%$. They used wavelengths ranging from 350-650 $\mathrm{nm}$. Stelzle et al. did not focus on the differentiation of nerve tissue and adipose tissue. ${ }^{67}$

The limitations of our study are the limited sample size and that we could not finally prove the chemical compositions of the extra peaks of IFAS in the HR-MAS NMR. For the optical absorption measurements, it was challenging to keep the tissue homogeneous throughout the length of the measurement cuvette. This inhomogeneity likely explains some of the variability seen in the data. The exact same tissue could not be compared for all cadavers and all measurement types. Up to now, it is unclear what effects freezing, thawing, and re-freezing the tissue had on the DRS and optical absorption measurements.

\section{Conclusions}

Histology, DRS spectra, light absorption, and HR-MAS NMR spectroscopy showed clear differences between fascicular nerve tissue and adipose tissues in an ex vivo human setting (Table 3). Collagen, water, and cellular nuclei concentration explain the difference between nerve fascicular tissue and the other tissues. Some differences observed between the optical absorption, DRS, and HR-NMR spectra of fascicular nerve tissue compared to the other tissues can't yet be explained and require further investigations in future studies. 


\section{References}

1. Ilfeld BM, Morey TE, Wright TW, Chidgey LK, Enneking FK. Continuous interscalene brachial plexus block for postoperative pain control at home: a randomized, double-blinded, placebo-controlled study. Anesth Analg. 2003;96(4):1089-95, table of contents.

2. Abdallah FW, Macfarlane AJ, Brull R. The Requisites of Needle-to-Nerve Proximity for Ultrasound-Guided Regional Anesthesia: A Scoping Review of the Evidence. Reg Anesth Pain Med. 2016;41(2):221-8.

3. Sites BD, Brull R, Chan VW, Spence BC, Gallagher J, Beach ML, et al. Artifacts and pitfall errors associated with ultrasound-guided regional anesthesia: Part II: A pictorial approach to understanding and avoidance. Reg Anesth Pain Med. 2010;35(2 Suppl):S81-92.

4. Gadsden J, McCally C, Hadzic A. Monitoring during peripheral nerve blockade. Curr Opin Anaesthesiol. 2010;23(5):656-61.

5. Hong JH, Huh B, Shin HH. Comparison Between Digital Subtraction Angiography and Real-time Fluoroscopy to Detect Intravascular Injection During Lumbar Transforaminal Epidural Injections. Reg Anesth Pain Med. 2014;39(4):329-32.

6. Lee MH, Yang KS, Kim YH, Jung HD, Lim SJ, Moon DE. Accuracy of live fluoroscopy to detect intravascular injection during lumbar transforaminal epidural injections. Korean J Pain. 2010;23(1):18-23.

7. Chien GC, Candido KD. Digital Subtraction Angiography is Not the Answer for Safe Epidural Injections. Pain Physician. 2014;17(3):E413-4.

8. O'Neil JMD. Musculoskeletal Ultrasound, Anatomy and Technique. 1 ed. New York: Spinger-Verlag New York; 2008. 348 p.

9. Gray AT. Ultrasound-guided regional anesthesia: current state of the art. Anesthesiology. 2006;104(2):368-73, discussion 5A.

10. Perlas A, Niazi A, McCartney C, Chan V, Xu D, Abbas S. The sensitivity of motor response to nerve stimulation and paresthesia for nerve localization as evaluated by ultrasound. Reg Anesth Pain Med. 2006;31(5):445-50.

11. Neal JM. Ultrasound-guided regional anesthesia and patient safety: An evidence-based analysis. Reg Anesth Pain Med. 2010;35(2 Suppl):S59-67.

12. Terkawi AS, Karakitsos D, Elbarbary M, Blaivas M, Durieux ME. Ultrasound for the anesthesiologists: present and future. ScientificWorldJournal. 2013 Nov 20;2013:683685.

13. Neuburger M, Schwemmer U, Volk T, Gogarten W, Kessler P, Steinfeldt T. [Localization of peripheral nerves. Success and safety with electrical nerve stimulation]. Anaesthesist. 2014;63(5):422-8.

14. Neal JM, Bernards CM, Hadzic A, Hebl JR, Hogan QH, Horlocker TT, et al. ASRA Practice Advisory on Neurologic Complications in Regional Anesthesia and Pain Medicine. Reg Anesth Pain Med. 2008;33(5):404-15.

15. Barrington MJ, Watts SA, Gledhill SR, Thomas RD, Said SA, Snyder GL, et al. Preliminary results of the Australasian Regional Anaesthesia Collaboration: a prospective audit of more than 7000 peripheral nerve and plexus blocks for neurologic and other complications. Reg Anesth Pain Med. 2009;34(6):534-41.

16. Jacob AK, Mantilla CB, Sviggum HP, Schroeder DR, Pagnano MW, Hebl JR. Perioperative nerve injury after total knee arthroplasty: regional anesthesia risk during a 20-year cohort study. Anesthesiology. 2011;114(2):311-7.

17. Auroy Y, Benhamou D, Bargues L, Ecoffey C, Falissard B, Mercier FJ, et al. Major complications of regional anesthesia in France: The SOS Regional Anesthesia Hotline Service. Anesthesiology. 2002;97(5):1274-80.

18. Orebaugh SL, Kentor ML, Williams BA. Adverse outcomes associated with nerve stimulator-guided and ultrasound-guided peripheral nerve blocks by supervised trainees: update of a single-site database. Reg Anesth Pain Med. 2012;37(6):577-82.

19. Barrington MJ, Snyder GL. Neurologic complications of regional anesthesia. Curr Opin Anaesthesiol. 2011;24(5):554-60.

20. Houten JK, Errico TJ. Paraplegia after lumbosacral nerve root block: report of three cases. Spine J. $2002 ; 2(1): 70-5$. 
21. Helen L, O'Donnell BD, Moore E. Nerve localization techniques for peripheral nerve block and possible future directions. Acta Anaesthesiol Scand. 2015;59(8):962-74.

22. Malhotra G, Abbasi A, Rhee M. Complications of transforaminal cervical epidural steroid injections. Spine (Phila Pa 1976). 2009;34(7):731-9.

23. Candido KD, Knezevic N. Cervical epidural steroid injections for the treatment of cervical spinal (neck) pain. Curr Pain Headache Rep. 2013;17(2):314.

24. Helm S, Glaser S, Falco F, Henry B. A medical-legal review regarding the standard of care for epidural injections, with particular reference to a closed case. Pain Physician. 2010;13(2):145-50.

25. Murthy NS, Maus TP, Behrns CL. Intraforaminal location of the great anterior radiculomedullary artery (artery of Adamkiewicz): a retrospective review. Pain Med. 2010;11(12):1756-64.

26. Huntoon MA, Martin DP. Paralysis after transforaminal epidural injection and previous spinal surgery. Reg Anesth Pain Med. 2004;29(5):494-5.

27. Kennedy DJ, Dreyfuss P, Aprill CN, Bogduk N. Paraplegia following image-guided transforaminal lumbar spine epidural steroid injection: two case reports. Pain Med. 2009;10(8):1389-94.

28. Auroy Y, Narchi P, Messiah A, Litt L, Rouvier B, Samii K. Serious complications related to regional anesthesia: results of a prospective survey in France. Anesthesiology. 1997;87(3):479-86.

29. Moayeri N, Bigeleisen PE, Groen GJ. Quantitative architecture of the brachial plexus and surrounding compartments, and their possible significance for plexus blocks. Anesthesiology. 2008;108(2):299-304.

30. Moayeri N, Groen GJ. Differences in quantitative architecture of sciatic nerve may explain differences in potential vulnerability to nerve injury, onset time, and minimum effective anesthetic volume. Anesthesiology. 2009;111(5):1128-34.

31. Sala-Blanch X, Ribalta T, Rivas E, Carrera A, Gaspa A, Reina MA, et al. Structural injury to the human sciatic nerve after intraneural needle insertion. Reg Anesth Pain Med. 2009;34(3): 201-5.

32. Bonnel F. Microscopic anatomy of the adult human brachial plexus: an anatomical and histological basis for microsurgery. Microsurgery. 1984;5(3):107-18.

33. Hendriks BH, Balthasar AJ, Lucassen GW, van der Voort M, Mueller M, Pully VV, et al. Nerve detection with optical spectroscopy for regional anesthesia procedures. J Transl Med. 2015;13:380.

34. Steinfeldt T, Graf J, Schneider J, Nimphius W, Weihe E, Borgeat A, et al. Histological Consequences of Needle-Nerve Contact following Nerve Stimulation in a Pig Model. Anesthesiol Res Pract. 2011;2011: 591851.

35. Bashkatov AN, Genina E.A., et al. Optical properties of the subcutaneous adipose tissue in the spectral range 400-2500 nm. Optics and Spectroscopy. 2005;99(5):836-42

36. Bashkatov AN, Genina E.A., et al. Optical properties of human skin, subcutaneous and muscous tissues in the wavelength range from 400 t2000 nm. Journal of Physics D-Applied Physics. 2005;38(15):2543-55.

37. Rathmell JP, Desjardins AE, van der Voort M, Hendriks BH, Nachabe R, Roggeveen S, et al. Identification of the epidural space with optical spectroscopy: an in vivo swine study. Anesthesiology. 2010;113(6): 1406-18.

38. Brynolf M, Sommer M, Desjardins AE, van der Voort M, Roggeveen S, Bierhoff W, et al. Optical detection of the brachial plexus for peripheral nerve blocks: an in vivo swine study. Reg Anesth Pain Med. 2011;36(4):350-7.

39. Desjardins AE, Hendriks BH, van der Voort M, Nachabe R, Bierhoff W, Braun G, et al. Epidural needle with embedded optical fibers for spectroscopic differentiation of tissue: ex vivo feasibility study. Biomed Opt Express. 2011;2(6):1452-61.

40. Desjardins AE, van der Voort M, Roggeveen S, Lucassen G, Bierhoff W, Hendriks BH, et al. Needle stylet with integrated optical fibers for spectroscopic contrast during peripheral nerve blocks. J Biomed Opt. 2011;16(7):077004.

41. Balthasar A, Desjardins AE, van der Voort M, Lucassen GW, Roggeveen S, Wang K, et al. Optical detection of vascular penetration during nerve blocks: an in vivo human study. Reg Anesth Pain Med. 2012;37(1):37.

42. Balthasar A, Desjardins AE, van der Voort M, Lucassen GW, Roggeveen S, Wang K, et al. Optical detection of peripheral nerves: an in vivo human study. Reg Anesth Pain Med. 2012;37(3):277-82. 
43. Schenkman KA, Marble, D. R., et al. Near infrared spectroscopic measurement of myoglobin oxygen saturation in the presence of hemboglobin using partial least-squares analysis. Applied Spectroscopy. 1999;53(3):325-31.

44. Kondepati VR, Heise HM, Backhaus J. Recent applications of near-infrared spectroscopy in cancer diagnosis and therapy. Anal Bioanal Chem. 2008;390(1):125-39.

45. Bydlon TM, Nachabe R, Ramanujam N, Sterenborg HJ, Hendriks BH. Chromophore based analyses of steady-state diffuse reflectance spectroscopy: current status and perspectives for clinical adoption. J Biophotonics. 2015;8(1-2):9-24.

46. Balthasar AJ, Lucassen GW, Sommer M, van der Voort M, Gijsbers G, Arnoldussen CW, et al. The Role of Spectral Tissue Sensing During Lumbar Transforaminal Epidural Injection. Reg Anesth Pain Med. 2016;41(4):520-6.

47. Pasarica M, Gowronska-Kozak B, Burk D, Remedios I, Hymel D, Gimble J, et al. Adipose tissue collagen VI in obesity. J Clin Endocrinol Metab. 2009;94(12):5155-62.

48. JA K. Histological and Histochemical Methods: Theory and Practice. 4th ed. UK: Scion; 2008.

49. Di Scipio F, Raimondo S, Tos P, Geuna S. A simple protocol for paraffin-embedded myelin sheath staining with osmium tetroxide for light microscope observation. Microsc Res Tech. 2008;71(7):497-502.

50. Sasaki T, Shono T, Hashiguchi K, Yoshida F, Suzuki SO. Histological considerations of the cleavage plane for preservation of facial and cochlear nerve functions in vestibular schwannoma surgery. J Neurosurg. 2009;110(4):648-55.

51. Romeis - Mikroskopische Technik. Mulisch MW, Ulrich, editor: Springer Berlin Heidelberg; 2015.

52. Tuchin V. Tissue Optics - Light Scattering Methods and Instruments for Medical Diagnosis. 2nd ed. Bellingham: SPIE; 2007.

53. Bargo PR, Prahl SA, Goodell TT, Sleven RA, Koval G, Blair G, et al. In vivo determination of optical properties of normal and tumor tissue with white light reflectance and an empirical light transport model during endoscopy. J Biomed Opt. 2005;10(3):034018.

54. Zonios G, Bassukas I, Dimou A. Comparative evaluation of two simple diffuse reflectance models for biological tissue applications. Appl Opt. 2008;47(27):4965-73.

55. Sun J, Fu K, Wang A, Lin AW, Utzinger U, Drezek R. Influence of fiber optic probe geometry on the applicability of inverse models of tissue reflectance spectroscopy: computational models and experimental measurements. Applied optics. 2006;45(31):8152-62.

56. Reif R, A'Amar O, Bigio IJ. Analytical model of light reflectance for extraction of the optical properties in small volumes of turbid media. Applied optics. 2007;46(29):7317-28.

57. Amelink A, Sterenborg HJ, Bard MP, Burgers SA. In vivo measurement of the local optical properties of tissue by use of differential path-length spectroscopy. Optics letters. 2004;29(10):1087-9.

58. Farrell TJ, Patterson MS, Wilson B. A diffusion theory model of spatially resolved, steady-state diffuse reflectance for the noninvasive determination of tissue optical properties in vivo. Med Phys. 1992;19(4):879-88.

59. Nachabe R, Hendriks BH, van der Voort M, Desjardins AE, Sterenborg HJ. Estimation of biological chromophores using diffuse optical spectroscopy: benefit of extending the UV-VIS wavelength range to include 1000 to 1600 nm. Biomed Opt Express. 2010;1(5):1432-42.

60. Nachabe R, Hendriks BH, Desjardins AE, van der Voort M, van der Mark MB, Sterenborg HJ. Estimation of lipid and water concentrations in scattering media with diffuse optical spectroscopy from 900 to 1,600 nm. J Biomed Opt. 2010;15(3):037015.

61. Nachabe R, Evers DJ, Hendriks BH, Lucassen GW, van der Voort M, Rutgers EJ, et al. Diagnosis of breast cancer using diffuse optical spectroscopy from 500 to $1600 \mathrm{~nm}$ : comparison of classification methods. J Biomed Opt. 2011;16(8):087010.

62. Paszek M, Weaver V. Biophysics. Enforcing order on signaling. Science. 2010;327(5971):1335-6.

63. Chapter 4. Myelin Formation SaB, Quarles. PMaRH. Chapter 4. Myelin Formation, Structure and Biochemistry Pierre Morell and Richard H Quarles. Philadelphia: Lippincott-Raven; 1999.

64. Stuart A. Fysh DAJS, and Peter M. Fredericks. Near-Infrared Diffuse Reflectance Spectroscopy of Coal. Apllied Spectroscopy. 1985;29(2):354-7. 
65. Ramstedt B, Slotte JP. Membrane properties of sphingomyelins. FEBS Lett. 2002;531(1):33-7.

66. Schols RM, ter Laan M, Stassen LP, Bouvy ND, Amelink A, Wieringa FP, et al. Differentiation between nerve and adipose tissue using wide-band (350-1,830 nm) in vivo diffuse reflectance spectroscopy. Lasers Surg Med. 2014;46(7):538-45.

67. Stelzle F, Zam A, Adler W, Tangermann-Gerk K, Douplik A, Nkenke E, et al. Optical nerve detection by diffuse reflectance spectroscopy for feedback controlled oral and maxillofacial laser surgery. J Transl Med. 2011;9:20. 


\section{Supplemental material}

\section{Supplement 4.1}

$2 \mathrm{D}^{13} \mathrm{C}-{ }^{1} \mathrm{H}$ HSQC spectra of soluble lipid fractions of: A. IFAS tissue extracted with $\mathrm{CDCl}_{3}{ }^{-}$ $\mathrm{MeOH}(2: 1 \mathrm{v} / \mathrm{v})$. After solvent evaporation on a Speedvac, the fatty acid content is redissolved in $\mathrm{CDCl} 3$. Peaks indicate a mixture of abundant phospholipids normally found in human fat tissue-namely, oleic acid 18:1(cis-9), linoleic acid 18:2 (cis-9,12), and stearic acid (18:0). NFAS and SCF exhibit similar types of HSQC spectra, showing the same abundant phospholipids. B. Insoluble fraction after $\mathrm{CDCl}_{3}-\mathrm{MeOH}$ extraction of IFAS tissue. This part contains white precipitates that separated out in between the water$\mathrm{MeOH}$ layer and chloroform layer during extraction. The thin layer of white precipitates was collected, washed with $\mathrm{CDCl}_{3}$, and followed by wash steps with water. After drying at room temperature underneath a flow of nitrogen gas, the white substance was redissolved in DMSO- $\mathrm{d}^{6}$ and studied by NMR. For comparison, reference 2D spectra of pure lipid compounds are compared: $C$. Saturated solution of sphingomyelin in $\mathrm{CDCl}_{3}$, and $\boldsymbol{D}$. Cholesterol $(30 \mathrm{mg} / \mathrm{ml})$ in $\mathrm{CDCl}_{3}$. The corresponding one-dimensional ${ }^{1} \mathrm{H}$ spectra of the four different samples are plotted on top of the 2D HSQC spectra. The asterisks in the $2 \mathrm{D}$ spectra indicate protons of residual solvents, $\mathrm{CDCl}_{3}$, and $\mathrm{DMSO}$, respectively. Arrows and rectangle boxes in plot $\mathrm{B}$ indicate new peaks of lipid components in the DMSO fraction that have limited solubility in chloroform and that become enriched relative to, for example, those in spectra shown in Figure S4.1A recorded for extracted tissue in $\mathrm{CDCl}_{3}$ (see also Figure S4.2).

\section{Supplement 4.2}

Comparison between the most obvious spectral differences in $2 \mathrm{D}^{13} \mathrm{C}-{ }^{-1} \mathrm{H} \mathrm{HSQC}$ spectra overlaid with NFAS (red color), IFAS (blue color), and SCF (yellow color) redissolved in $\mathrm{CDCl}_{3}$ after extraction with $\mathrm{CDCl}_{3}-\mathrm{MeOH}(2: 1 \mathrm{v} / \mathrm{v})$. A. Region containing aromatic resonances (rectangle box) that are predominantly found in IFAS and partially in NFAS but absent in SCF. B. Additional cross-peak of a molecule containing a possible choline group (rectangle box) that is only observed in NFAS and SCF extracts but not in IFAS. 
What is the optical signature of nerve tissue?
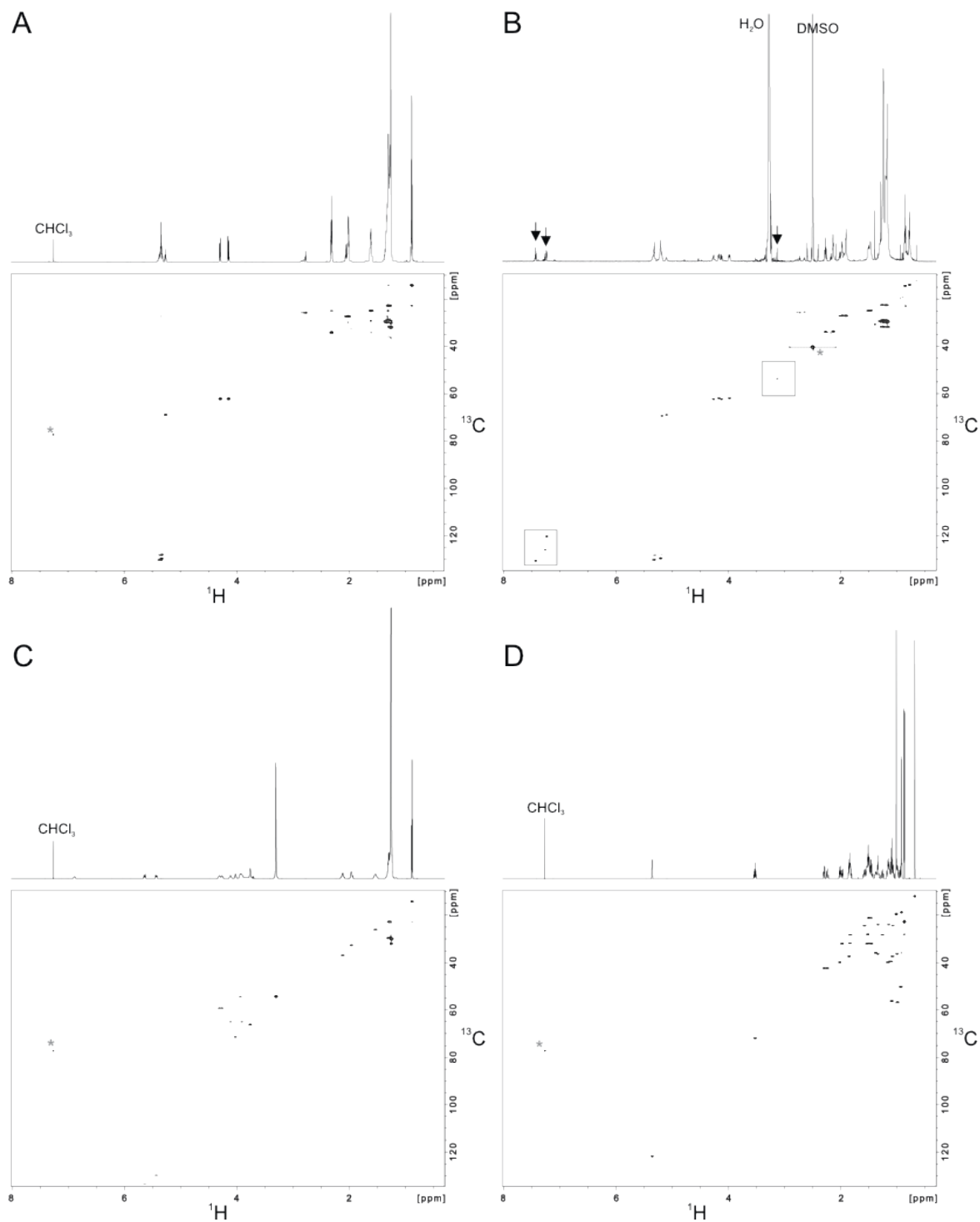

D

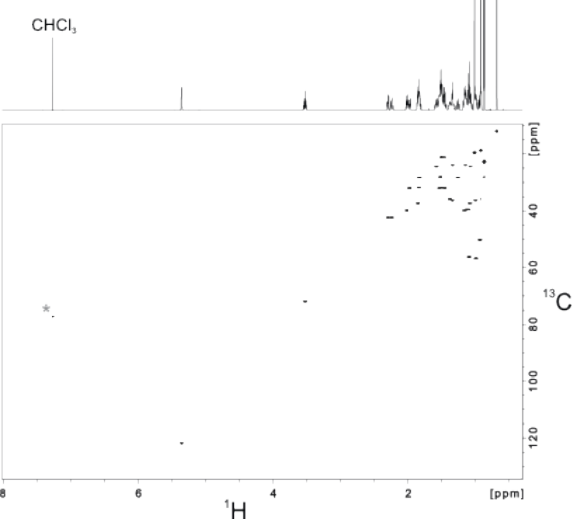

Figure S4.1 
Chapter 4

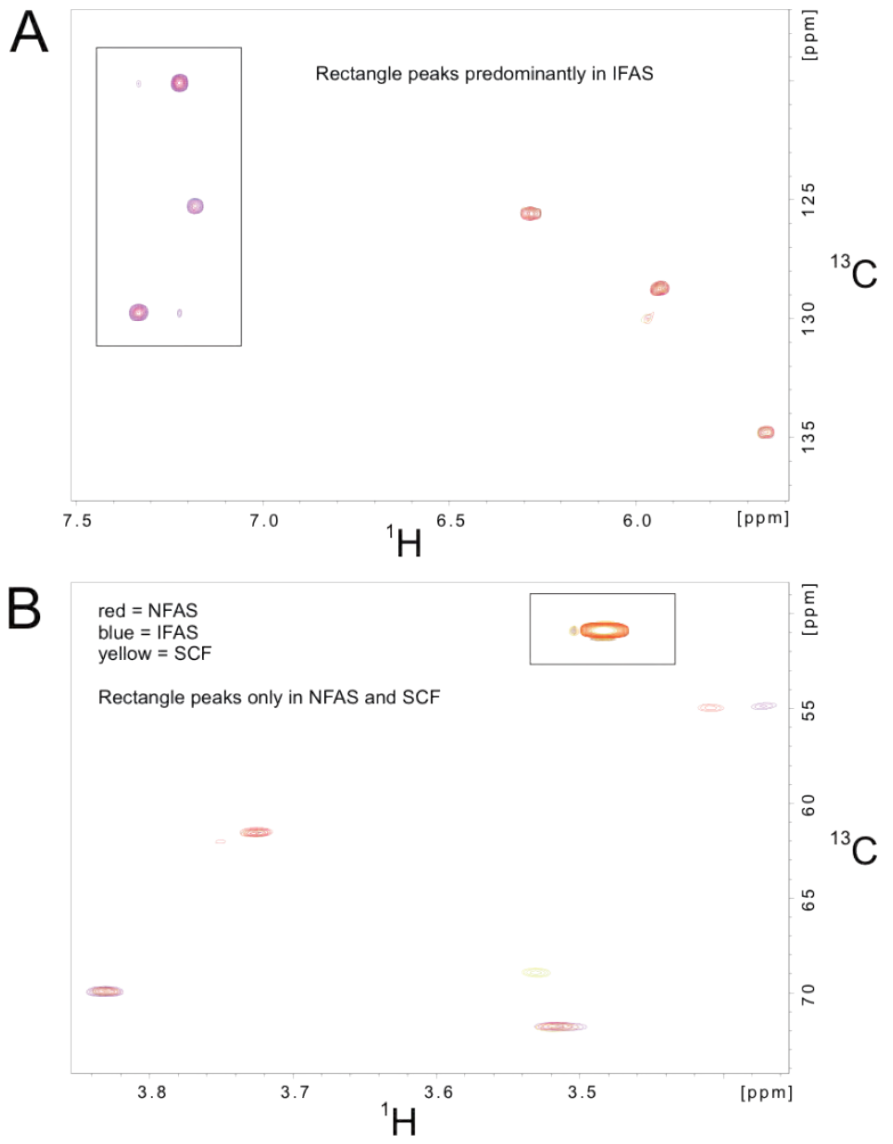

Figure $\$ 4.2$ 




\section{Chapter \\ Is it possible to detect tissue transitions with the STS system during peripheral nerve blocks?}

Optical detection of peripheral nerves An in vivo human study

Andrea Balthasar, Adrien E. Desjardins, Marjolein van der Voort, Gerald W. Lucassen, Stefan Roggeveen, Ke Wang, Walter Bierhoff, Alfons G.H. Kessels, Maarten van Kleef, Micha Sommer Reg Anesth Pain Med 2012;37:277-82 


\section{Abstract}

\section{Background and objectives}

A critical challenge encountered in interventional pain medicine procedures is to accurately and efficiently identify transitions to peripheral nerve targets. Current methods, which include ultrasound guidance and nerve stimulation, are not perfect. In this pilot study, we investigated the feasibility of identifying tissue transitions encountered during insertions toward peripheral nerve targets using optical spectroscopy.

\section{Methods}

Using a custom needle stylet with integrated optical fibers, ultrasound-guided insertions toward peripheral nerves were performed in 20 patients, with the stylet positioned in the cannula of a 20-gauge stimulation needle. Six different peripheral nerves were represented in the study, with 1 insertion per patient. During each insertion, optical reflectance spectra were acquired with the needle tip in subcutaneous fat, skeletal muscle, and at the nerve target region. Differences in the spectra were quantified with 2 parameters that provide contrast for lipid and hemoglobin, respectively.

\section{Results}

The transition of the needle tip from subcutaneous fat to muscle was associated with lower lipid parameter values $(P=0.003)$ and higher hemoglobin parameter values $(P=0.023)$. The transition of the needle tip from the muscle to the nerve target region was associated with higher lipid parameter values $(P=0.008)$.

\section{Conclusions}

The results indicate that the spectroscopic information provided by the needle stylet could potentially allow for reliable identification of transitions from subcutaneous fat to skeletal muscle and from the muscle to the nerve target region during peripheral nerve blocks. 


\section{Introduction}

Accurately and efficiently identifying nerves during interventional pain medicine procedures is crucial to maximize the likelihood of a positive outcome. The limitations of today's methods have been well documented. Nerve stimulation requires a muscle or sensory response that may not always be elicited, even when the needle tip is near a nerve. ${ }^{1}$ With ultrasound image guidance, directly visualizing nerves can be challenging. For instance, nerves may not be directly resolvable when they are small and in deep locations; artifacts such as acoustic shadowing and acoustic enhancement can hide important structures or distort normal anatomy. ${ }^{2,3}$ While there is a dearth of outcome statistics available in the literature, recent studies provide evidence that improvements in the accuracy at which needles are positioned have corresponding improvements in patient outcomes. For instance, in a study of axillary brachial plexus blocks, the observed success rate was $63 \%$ when nerve stimulation was used alone and $81 \%$ when both ultrasound and nerve stimulation were used in combination. ${ }^{4}$ It is of great interest to develop more reliable and accurate methods with which to identify nerves.

Optical reflectance spectroscopy is a sensing modality that provides information about the molecular composition of tissue. The essential principle is that light is delivered to tissue and the reflected light is spectrally resolved. A spectrum acquired from a white surface that reflects light equally at all wavelengths has uniform intensities. Spectra that are acquired from biological tissue have nonuniform intensities owing to the wavelength dependencies of optical scattering and absorption. While optical scattering tends to manifest as gradual changes in intensity with wavelength, optical absorption can result in significantly lower intensities in narrow wavelength ranges, which are termed absorption peaks. Absorption peaks in the visible wavelength range derived in large part from optical absorption by hemoglobin. Lipids and water contribute prominently to absorption in the infrared wavelength range.

Recently, several studies have demonstrated that reflectance spectroscopy has a strong potential to provide real-time guidance during interventional pain and regional anesthesia procedures. To acquire spectra from tissues at the needle tip, custom needles were developed in which optical fibers were integrated into the needle cannula or stylet. ${ }^{5-9}$ In an in vivo study of ultrasound guided insertions toward the axillary brachial plexus in swine, differences in spectra corresponding to transitions of the needle tip from 1 tissue type to another were quantified with parameters that provide contrast for optical absorption by hemoglobin and lipid, respectively. The transition of the needle tip from the skeletal muscle to the nerve target region was associated with higher lipid parameter values and lower hemoglobin parameter values; the transition of the needle tip from muscle fascia to the nerve target region was associated with higher lipid parameter values. ${ }^{9}$

The objective of this pilot study was to investigate the potential of optical reflectance spectroscopy to differentiate between tissue transitions encountered during 
insertions toward peripheral nerves in humans during interventional pain management procedures.

\section{Methods}

\section{Patients enrollment}

The study was conducted at Maastricht University Medical Centre (Maastricht, the Netherlands) with medical ethics committee approval (Medisch Ethische Commissie Academisch Ziekenhuis Maastricht, Maastricht, the Netherlands). A total of 20 patients (7 women and 13 men; age range, 25-77 years) were included in the study. All patients provided written informed consent for their participation. Each patient was scheduled to receive a nerve block that comprised 1 needle insertion. The blocks were performed either for diagnostic purposes and carried out using local anesthetics alone (levobupivacaine $0.75 \%$ ) or for the treatment of mononeuropathic pain using local anesthetics (levobupivacaine $0.75 \%$ ) and corticosteroids. The targets included the ilioinguinal nerve ( 8 insertions), the lateral femoral cutaneous nerve ( 5 insertions), the peroneal nerve ( 3 insertions), the femoral nerve ( 2 insertions), the sural nerve ( 1 insertion), and the median nerve ( 1 insertion). All procedures were performed without sedation.

\section{Optical stylet and console}

The custom optical stylet, developed by Philips Research (Eindhoven, the Netherlands) and InVivo GmbH (Schwerin, Germany) and CE-marked by InVivo GmbH, was compatible with a commercially available, 20-gauge radiofrequency needle cannula (Neurotherm, Amsterdam, the Netherlands). The needle cannula had a length of $100 \mathrm{~mm}$ and a bevel angle of 20 degrees. Two optical fibers with core diameters of $100 \mathrm{Km}$ were integrated in the needle stylet; the distance between the centers of the fiber cores was $0.9 \mathrm{~mm}$, as measured along the bevel surface. One fiber delivered broadband light to the tissue; the other received scattered light and directed it to the optical console. The optical console, which was previously described in detail, ${ }^{10}$ consisted of a tungstenhalogen light source and 2 spectrometers that resolved received light from tissue across the wavelength range of 500 to $1600 \mathrm{~nm}$. The optical console and stylet are shown in Figure 5.1.

\section{Insertion protocol}

Ultrasound imaging (iU22; Philips Healthcare, Bothell, Washington) with a linear array, 5- to $12-\mathrm{MHz}$ probe (L12-5) was used to guide all insertions, with the needle visualized in-plane. All insertions were performed by 1 practitioner (M.S.). The insertion tech- 
niques that were used are described by Eichenberger et al. ${ }^{11}$ for ilioinguinal nerve blocks, Hurdle et al. ${ }^{12}$ for lateral femoral cutaneous nerve blocks, Narouze ${ }^{13}$ for median and peroneal nerve blocks, Marhofer et al. ${ }^{14}$ for femoral nerve blocks, and Redborg et al. ${ }^{15}$ for sural nerve blocks.

During each insertion, the needle tip was held in place at 2 or 3 different locations: subcutaneous fat, skeletal muscle (when accessible), and the nerve target region. At each location, 5 tissue spectra and 5 background spectra were acquired, with each spectrum acquired in 0.5 seconds. The latter spectra were acquired with the light source shuttered to measure the contribution of ambient light. In parallel with the acquisition of spectra, a 3-second ultrasound video was acquired. Subsequently, the practitioner provided a score to indicate the confidence with which he identified the type of tissue where the needle tip resided. The scores ranged from 1 (completely uncertain) to 3 (completely certain). At all 3 locations, it was ensured that the needle tip was clearly visible with ultrasound. When the needle tip was positioned at the location identified ultrasonically as the nerve target region, electrical stimulation was performed and the threshold for eliciting a sensory response was recorded (all elicited responses were sensory). The anesthetic was injected at the nerve target location after the spectra were acquired. The optical spectra remained hidden to the practitioner during the insertions to ensure that they would not influence the position of the needle tip.
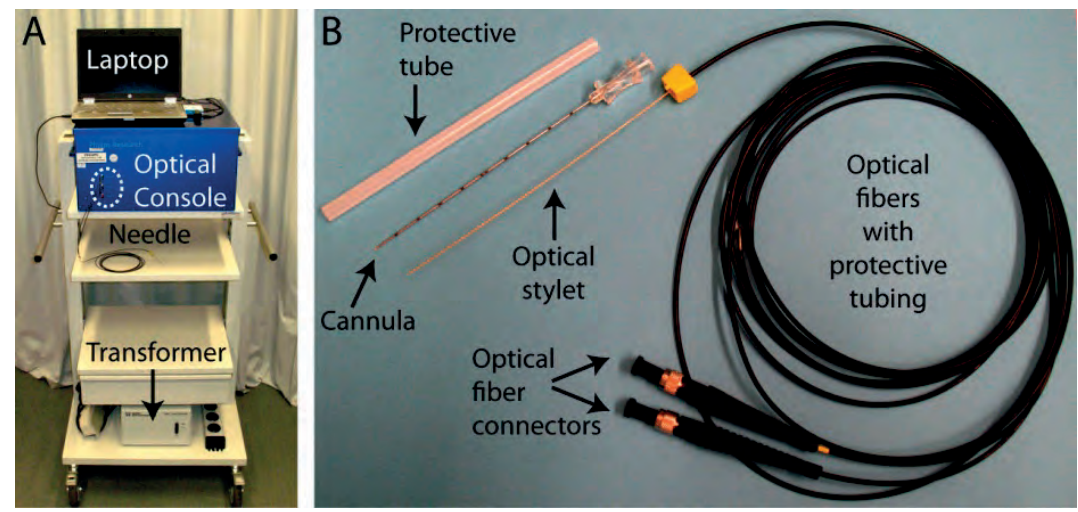

Figure 5.1 A) The optical console, which comprises a light source and 2 spectrometers, was controlled by the laptop positioned above it. The attachment points on the console for the 2 optical fiber connectors in the optical stylet are indicated with the dashed oval. Power was provided to the optical console and laptop via an isolation transformer. B) The optical stylet, with 2 integrated optical fibers, was designed to be compatible with a conventional, 20-gauge needle cannula. The protective tube covers the cannula during transport. Outside the stylet, the optical fibers were protected with tubing; they terminated at 2 SubMiniature version A (SMA) optical fiber connectors. 


\section{Spectral analysis}

Spectral analysis was performed offline with custom software written in MATLAB (MathWorks, Natick, Massachusetts). Three preprocessing steps were performed: (a) subtraction of the mean background spectrum, (b) intensity calibration with a spectrum that was acquired from a white reflectance standard using a needle distinct from those used in the study, and (c) normalization with respect to the mean intensities. Subsequently, 2 empirical parameters were calculated from each spectrum: a lipid parameter and a hemoglobin parameter. As described in a previous study, ${ }^{9}$ the lipid parameter, $\mathrm{L}$, was defined to be equal to the difference in spectrum intensity, $\mathrm{S}$, at 2 wavelengths, normalized by the spectrum intensity at the first wavelength:

$$
L=[S(1195 \mathrm{~nm})-\mathrm{S}(1210 \mathrm{~nm})] / \mathrm{S}(1195 \mathrm{~nm}) .
$$

At the first wavelength of $1195 \mathrm{~nm}$, optical absorption of water and lipid is equal; at the second wavelength of $1210 \mathrm{~nm}$, optical absorption of lipid is at a local maximum. The hemoglobin parameter is defined in a similar fashion, with wavelengths at which optical absorption is equal for both oxygenated and deoxygenated forms:

$$
H=[S(797 n m)-S(545 n m)] / S(797 n m) .
$$

An increase in the lipid and hemoglobin parameters is consistent with an increase in the lipid and hemoglobin content of tissue from which the spectrum derives, respectively, provided that optical scattering is constant. We note that these empirical parameters are dimensionless (i.e., they do not have associated units) and that they are not concentration measurements; they can assume both positive and negative values. Parameters obtained from the 5 spectra acquired from each position were averaged.

\section{Statistical analysis}

Differences between the averaged parameter values obtained from pairs of tissue types were analyzed with the Wilcoxon signed-rank nonparametric test (2-sided). The 3 pairs of tissue types were (a) the subcutaneous fat and the skeletal muscle, (b) the skeletal muscle and the nerve target region, and (c) the subcutaneous fat and the nerve target region. This test was applied separately to differences in lipid parameter values and hemoglobin parameter values. Statistical significance was accepted at the level of $P<0.05$.

For inclusion in the statistical analyses, the following 2 criteria were imposed on each set of spectra that were acquired during an insertion: the needle tip position in subcutaneous fat and skeletal muscle was identified ultrasonically with a certainty level of 2 or 3; at the nerve target region, a sensory response was elicited electrically at a threshold lower than $0.5 \mathrm{~mA}$. 


\section{Results}

Spectral data sets from 15 of the 20 insertions satisfied the criteria for inclusion in statistical analyses. In 3 of the 5 other insertions, a sensory response could not be elicited. In these 3 insertions, needle tip positioning in the nerve target region was twice determined ultrasonically with a certainty level of 3 and once with a certainty level of 1 ; all needle positioning in subcutaneous fat and skeletal muscle was determined ultrasonically with a certainty level of 3 . The remaining 2 spectral data sets did not satisfy the inclusion criteria because spectra from skeletal muscle could not be obtained.

Ultrasound images, optical spectra, and the corresponding spectral parameters obtained from 1 insertion to the ilioinguinal nerve are shown in Figure 5.2. Needle tip positioning in the nerve target region was confirmed with electrical stimulation (threshold $<0.5 \mathrm{~mA}$ ); in the subcutaneous fat, the skeletal muscle, and the nerve target region, it was determined ultrasonically with a certainty level of 3. The transition from the skeletal muscle to the nerve target region was associated with a higher lipid parameter and a lower hemoglobin parameter. The highest lipid parameter was obtained from the subcutaneous fat; the highest hemoglobin parameter was obtained from the muscle.

A transition from subcutaneous fat to skeletal muscle was associated with a negative mean difference in the lipid parameter (mean [SD], -3.2 [2.6]) and a positive mean difference in the hemoglobin parameter (mean [SD], 23 [34]), as shown in Figure 5.3. The differences in the lipid parameters obtained from these 2 tissue types were negative in 14 of the 15 insertions; the corresponding differences in the hemoglobin parameters were positive in 13 of the 15 insertions. Both of these differences were statistically significant (lipid parameter, $\mathrm{P}=0.003$; hemoglobin parameter, $\mathrm{P}=0.023$ ).

A transition of the needle tip from the skeletal muscle to the nerve target region was associated with a positive mean difference in the lipid parameter (mean [SD], 2.2 [3.0]) and a negative mean difference in the hemoglobin parameter (mean [SD], -20 [40]). The differences in the lipid parameter obtained from these 2 tissue types were positive in 14 of the 15 insertions (Figure 5.3); the corresponding differences in the hemoglobin parameter were negative in 10 of the 15 insertions. Only the differences in the lipid parameter were statistically significant (lipid parameter, $\mathrm{P}=0.008$; hemoglobin parameter, $\mathrm{P}=0.069)$.

A mixed picture was observed for transitions from subcutaneous fat to the nerve target region. The mean difference was negative for the lipid parameter (mean [SD], 1.0 [3.5]) and positive for the hemoglobin parameter (mean [SD], 3.5 [35]). The differences in the lipid parameter were negative in 11 of the 15 insertions; the corresponding differences in the hemoglobin parameters were positive in 6 of the 15 insertions. Neither difference was statistically significant (lipid parameter, $P=0.334$; hemoglobin parameter, $\mathrm{P}=0.955)$. 

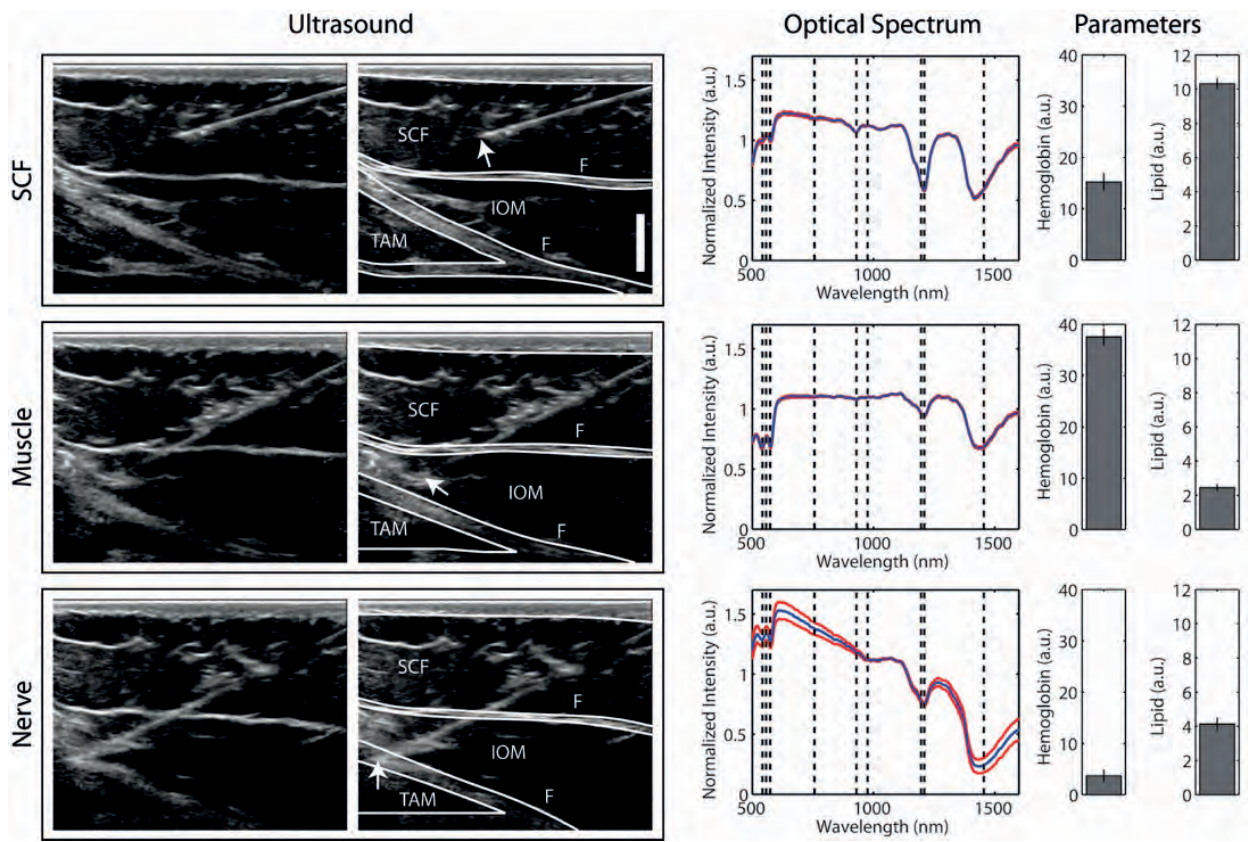

Figure 5.2 Ultrasound images and optical spectra acquired from a single insertion to the ilioinguinal nerve. Ultrasound images were acquired with the needle tip in subcutaneous fat (top), in skeletal muscle (middle), and at the nerve target region (bottom). Each ultrasound image is presented twice, with selected anatomical features identified on the rightmost image. Scale bar, $5 \mathrm{~mm}$. F indicates muscle fascia; IOM, internal oblique muscle; SCF, subcutaneous fat; TAM, transverse abdominal muscle. The arrows indicate the locations of the needle tip. To the right of the ultrasound image, the corresponding optical spectra are presented. The optical spectra indicate the intensity of light received by the optical stylet (linear units) as a function of the wavelength, normalized by the mean intensity (a.u., arbitrary units). Mean spectral values (blue) and SDs relative to the mean (red) are displayed as a function of wavelength.

Specific wavelengths corresponding to selected absorption peaks are indicated with dashed lines. Oxyhemoglobin, 542 and $574 \mathrm{~nm}$; deoxyhemoglobin, 557 and $757 \mathrm{~nm}$; lipids, 930 and 1210 $\mathrm{nm}$; water, 976, 1197, and $1455 \mathrm{~nm}$. To the far right, derived hemoglobin and lipid parameters from the needle positions that were identified on the ultrasound images are shown (mean [SD]).

In all 3 insertions in which electrical stimulation did not elicit a sensory response, transitions between skeletal muscle and the nerve target region were associated with increases in the lipid parameter (0.17 in the first insertion, 2.2 in the second, and 1.3 in the third) and decreases in the hemoglobin parameter (-17, -45 , and -35 , respectively). A mixed picture was observed for transitions between the subcutaneous fat and the nerve target region, with increases and decreases in both the lipid parameter ($2.0,-0.17$, and 0.22 , respectively) and the hemoglobin parameter $(20,-27$, and 26 , respectively). 


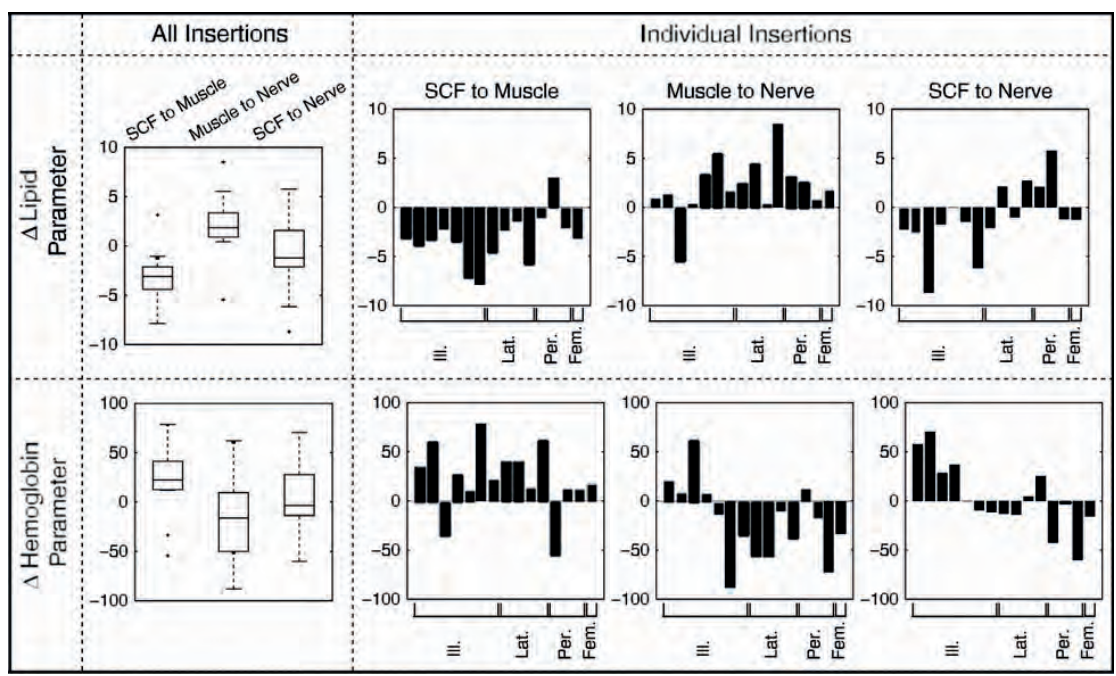

Figure 5.3 Differences in lipid parameters (top) and hemoglobin parameters (bottom) that correspond to transitions of the needle tip between 2 tissue types. For the transition designated as "SCF to Muscle," the difference (\$) in the lipid parameter for an individual insertion is calculated as the difference between the 2 lipid parameters obtained from the skeletal muscle and the subcutaneous fat, respectively. Equivalent calculations are performed for the transitions designated as "Muscle to Nerve" (skeletal muscle to nerve target region) and "SCF to Nerve" (subcutaneous fat to nerve target region) and for the hemoglobin parameters. On the left, differences in parameters from all insertions are combined as a box plot (bottom and top box, 25th and 75th percentiles; middle band, 50th percentile); points are drawn as outliers if they are larger than $\mathrm{Q}_{3}+$ $1.5\left(Q_{3}-Q_{1}\right)$ or smaller than $Q_{1}-1.5\left(Q_{3}-Q_{1}\right)$, where $Q_{1}$ and $Q_{3}$ are the 25 th and 75 th percentiles, respectively. On the right, the mean differences in parameters from individual insertions are grouped according to the type of target nerve. Fem indicates femoral; III, ilioinguinal; Lat, lateral femoral cutaneous; Per, peroneal.

\section{Discussion}

Information about the optical reflectivity of tissue has traditionally been unavailable during percutaneous interventions. Tissue color as perceived by the naked eye provides limited information about optical reflectivity, but it is nonetheless relevant for differentiating between tissues that are visible (e.g., during dissections and open surgeries). The optical reflectance spectra acquired with the needle stylet in this study provided significantly more information about tissue color than that perceived by the naked eye. They spanned both visible and near-infrared wavelength ranges, with much finer spectral resolution that allowed for the optical absorption signatures of hemoglobin, lipid, and water. This study provided initial indications that optical reflectance spectra acquired with a needle stylet are relevant for detecting tissue transitions encountered during peripheral nerve blocks in human patients. In future studies, the lipid and hemoglobin 
parameters that were used for detecting transitions could be calculated and displayed in real time.

Spectroscopic detection of peripheral nerves could have several potential advantages. First, optical reflectance spectroscopy is a direct method to provide information about tissue composition. The lipid parameter obtained from optical reflectance spectra provides contrast for lipids that may be found outside the nerve (e.g., in fibrofatty connective tissue) or inside the nerve (e.g., extrafascicular adipose tissue and in myelin sheaths).

The hemoglobin parameter provides contrast that could indicate differences in tissue vascularity. A second potential advantage of optical reflectance spectroscopy is that it could allow for the detection of transitions to vascular structures that are at risk for penetration; indeed, our research team recently provided indications that percutaneous access to an arterial lumen can be detected with optical reflectance spectroscopy. ${ }^{9}$ Optical reflectance spectroscopy might therefore decrease the risk of unintentional intravascular injections.

Several types of variations in the lipid and hemoglobin content of tissues may have contributed to the differences in parameter values that were observed in this study. Interpatient variations may arise in part from the age or obesity level of patients; both factors are known to correlate with the lipid content of skeletal muscle. ${ }^{16}$ Likewise, for a given nerve type, the amount of perineural fat can be expected to vary among patients. Tissue heterogeneities could give rise to intertissue differences in parameter values; within a particular tissue, for instance, the presence of a vascular or lymphatic structure close to the needle tip could affect the optical reflectance spectra. Accumulations of blood at the bevel surface may also influence blood parameter values; the extent to which this occurs may vary, as the needle is inserted through tissue and variations in tissue vascularity are encountered.

Of the 3 tissue transitions considered in this study, those from the subcutaneous fat to the skeletal muscle and from the skeletal muscle to the nerve target region could be detected with the highest accuracy. The comparatively lower accuracy with which transitions from the subcutaneous fat to the nerve target region could be detected likely reflects similarities in molecular composition; the presence of adipocytes and fibrofatty connective tissue can be expected in both tissue types. These results suggest that spectroscopic detection of the nerve target region may be more beneficial for insertions that involve transitions to the nerve target region from skeletal muscle than for those that involve transitions to the nerve target region via subcutaneous fat.

This study left several questions unanswered. First, it is unclear how the accuracy at which nerve target regions can be identified varies with the type of nerve. This accuracy may depend on the trajectory through tissue that is taken by the needle tip; as the results of this study suggested, transitions to nerve target regions via skeletal muscle may give rise to more distinctive changes in hemoglobin and lipid parameters than transitions from subcutaneous fat. Larger studies with multiple insertions for each 
nerve type are required to address this question and to measure correlations between parameter values and patient characteristics (e.g., age and variations in body mass composition). Second, it is not yet clear to what extent optical reflectance spectroscopy could improve patient outcomes. In this pilot study, the combination of electrical stimulation and ultrasound was used as a criterion standard with which to identify the nerve target region, so this question could not be directly addressed. Electrical stimulation and optical spectroscopy provide different information, as evidenced by the observation that a transition from skeletal muscle to the nerve target region gave rise to increases in the lipid parameter that are similar whether a sensory response was elicited by electrical stimulation. Future studies could compare patient outcomes when identification is based on electrical stimulation with those outcomes where identification is based on optical reflectance spectroscopy.

This was, to our knowledge, the first study to investigate the use of optical reflectance spectroscopy for detecting tissue transitions encountered during peripheral nerve blocks in human patients. The results suggest that transitions from the skeletal muscle to the nerve target region and from the subcutaneous fat to skeletal muscle could be reliably detected with parameters that provide contrast for optical absorption by lipids and hemoglobin. This study highlighted the potential of optical reflectance spectroscopy to reduce uncertainties associated with needle placement during ultrasound-guided insertions and thereby to improve the outcomes of peripheral nerve blocks. 


\section{References}

1. Urmey WF, Stanton J. Inability to consistently elicit a motor response following sensory paresthesia during interscalene block administration. Anesthesiology. 2002;96:552-4.

2. Marhofer $P$, Chan VWS. Ultrasound-guided regional anesthesia. Current concepts and future trends. Anesth Analg. 2007;104:1265-9.

3. Sites BD, Brull R, Chan VWS, et al. Artifacts and pitfall errors associated with ultrasound-guided regional anesthesia. Reg Anesth Pain Med. 2010;35:S81-92.

4. Chan VWS, Perlas A, McCartney CJL, Brull R, Xu DQ, Abbas S. Ultrasound guidance improves success rate of axillary brachial plexus block. Can J Anaesth. 2007;54:176-82.

5. Ting CK, Tsou MY, Chen PT, et al. A new technique to assist epidural needle placement: fiberoptic-guided insertion using two wavelengths. Anesthesiology. 2010;112:1128-35.

6. Rathmell JP, Desjardins AE, van der Voort M, et al. Identification of the epidural space with optical spectroscopy: an in vivo swine study. Anesthesiology. 2010;113:1406-18.

7. Desjardins $A E$, Hendriks BHW, van der Voort M, et al. Epidural needle with embedded optical fibers for spectroscopic differentiation of tissue: ex vivo feasibility study. Biomed Opt Express. 2011;2:1452-61.

8. Desjardins $A E$, van der Voort $M$, Roggeveen $\mathrm{S}$, et al. Needle stylet with integrated optical fibers for spectroscopic contrast during peripheral nerve blocks. J Biomed Opt. 2011;16:077004.

9. Brynolf M, Sommer M, Desjardins AE, et al. Optical detection of the brachial plexus for peripheral nerve blocks: an in vivo swine study. Reg Anesth Pain Med. 2011;36:350-357.

10. Nachabe' R, Hendriks BHW, Desjardins AE, van der Voort M, van der Mark MB, Sterenborg JCM. Estimation of lipid and water concentrations in scattering media with diffuse optical spectroscopy from 900 to 1600 nm. J Biomed Opt. 2010;15:037015.

11. Eichenberger U, Greher M, Kirchmair L, Curatolo M, Moriggl B. Ultrasound-guided blocks of the ilioinguinal and iliohypogastric nerve: accuracy of a selective new technique confirmed by anatomical dissection. Br J Anaesth. 2006;97:238-43.

12. Hurdle MF, Weingarten TN, Crisostomo RA, Psimos C, Smith J. Ultrasound-guided blockade of the lateral femoral cutaneous nerve: technical description and review of 10 cases. Arch Phys Med Rehabil. 2007;88:1362-4

13. Narouze SN. Atlas of Ultrasound-Guided Procedures in Interventional Pain Management. 1st ed. New York, NY: Springer; 2010.

14. Marhofer P, Schro" gendorfer K, Wallner T, Koinig H, Mayer N, Kapral S. Ultrasonographic guidance reduces the amount of local anesthetic for 3-in-1 blocks. Reg Anesth Pain Med. 1998;23:584-8.

15. Redborg KE, Sites BD, Chinn CD, et al. Ultrasound improves the success rate of a sural nerve block at the ankle. Reg Anesth Pain Med. 2009;34:24-8.

16. Gallagher D, Kuznia P, Heshka S, et al. Adipose tissue in muscle: a novel depot similar in size to visceral adipose tissue. Am J Clin Nutr. 2005;81:903-10. 




\section{Chapter}

\section{Can the most appropriate and the safest injection environment be determined with the STS system during lumbar transforaminal epidural injections?}

The role of spectral tissue sensing during lumbar transforaminal epidural injection

Andrea J.R. Balthasar, Gerald W. Lucassen, Micha Sommer, Marjolein van der Voort, Geert Gijsbers, Carsten W.K.P.

Arnoldussen, Alfons Kessel, Stefan Roggeveen, Arno Lataster, Maarten van Kleef Reg Anesth Pain Med 2016;41(4):520-6 


\section{Abstract}

Spectral tissue sensing (STS) exploits the scattering and absorption of light by tissue. The main objective of the present study was to determine whether STS can discriminate between correct and incorrect placement of the needle tip during lumbar transforaminal epidural injection. This was a single-blind prospective observational study in 30 patients with lumbar radicular pain scheduled for lumbar transforaminal epidural injection.

Spectral tissue sensing data from the needle tip were acquired along the needle trajectory at 4 predefined measurement points and compared with ultrasound, fluoroscopy, and digital subtraction angiography images. Spectral tissue sensing data contained the full spectra. The lipid and hemoglobin content at the different measurement points was also calculated, and partial leastsquares discriminant analysis was used to estimate the sensitivity and specificity of STS.

Spectral tissue sensing identified correct needle placement with a sensitivity of $57 \%$ and a specificity of $82 \%$, and intraforaminal versus extraforaminal locations were identified with a sensitivity of $80 \%$ and a specificity of $71 \%$. 


\section{Introduction}

Two factors are critical to the safety and technical success of lumbar transforaminal epidural injections (LTEIS): needle placement, which should be close to but not inside the nerve, and avoiding an intravascular needle-tip position. The needle can be placed close to a predetermined location using anatomical markers in combination with various imaging techniques such as fluoroscopy, ultrasound, and digital subtraction angiography (DSA). However, all of these techniques have limitations. Fluoroscopy combined with DSA is commonly used in LTEIs. ${ }^{1}$ Nevertheless, fluoroscopic needle guidance does not prevent nerve or vascular puncture or intraneural injection. The incidence of intravascular injection or simultaneous epidural and vascular injection during lumbar intraforaminal epidural injections is $6.1 \%$ to $11.3 \%$. $^{2,3}$ The sensitivity of real-time fluoroscopy in detecting intravascular needle placement is $71 \%$ compared with DSA.

Reports of complications and adverse effects of transforaminal epidural injections with particulate steroids such as spinal cord and brain infarctions can be attributed to the limitations of current guidance techniques. ${ }^{4}$ It may be possible to improve the accuracy of needle placement by using additional information about the tissue type at the site of the needle tip. Philips Research (Eindhoven, the Netherlands) has developed a system based on spectral tissue sensing (STS), which uses optical spectroscopy (500$1600 \mathrm{~nm}$ ) to provide information about tissue composition (lipid, water, blood content) in front of the needle tip.

Spectral tissue sensing is based on diffuse reflectance spectroscopy. ${ }^{5}$ It exploits the fact that the scattering and absorption of light by tissue are determined by the tissue composition., 5 Studies have been reported on the use of STS in swine and in patients. $^{7-10}$ This study extends previous research by investigating STS in a larger, more homogenous patient group. The main objective was to test the hypothesis that STS can discriminate between correct (i.e., safe) and incorrect placement of the needle tip during LTEI.

\section{Methods}

\section{Treatment procedure and data collection}

All treatments were performed according to the scheme presented in Figure 6.1, using fluoroscopic guidance (Ziehm Vision mobile X-ray imaging system; Ziehm, Nürnberg, Germany) and ultrasound (iU22, 5- to 12-MHz probe; Philips Healthcare, Eindhoven, the Netherlands). One physician (A.J.R.B.) performed all insertions and operated the ultrasound machine. Injections were made using 100- or 144-mm long needles, 20-gauge SMK (NeuroTherm, Amsterdam, the Netherlands) with an integrated optical stylet and 
an intravenous T-connector. Insertions were performed using the technique described by van Boxem et al. ${ }^{11}$

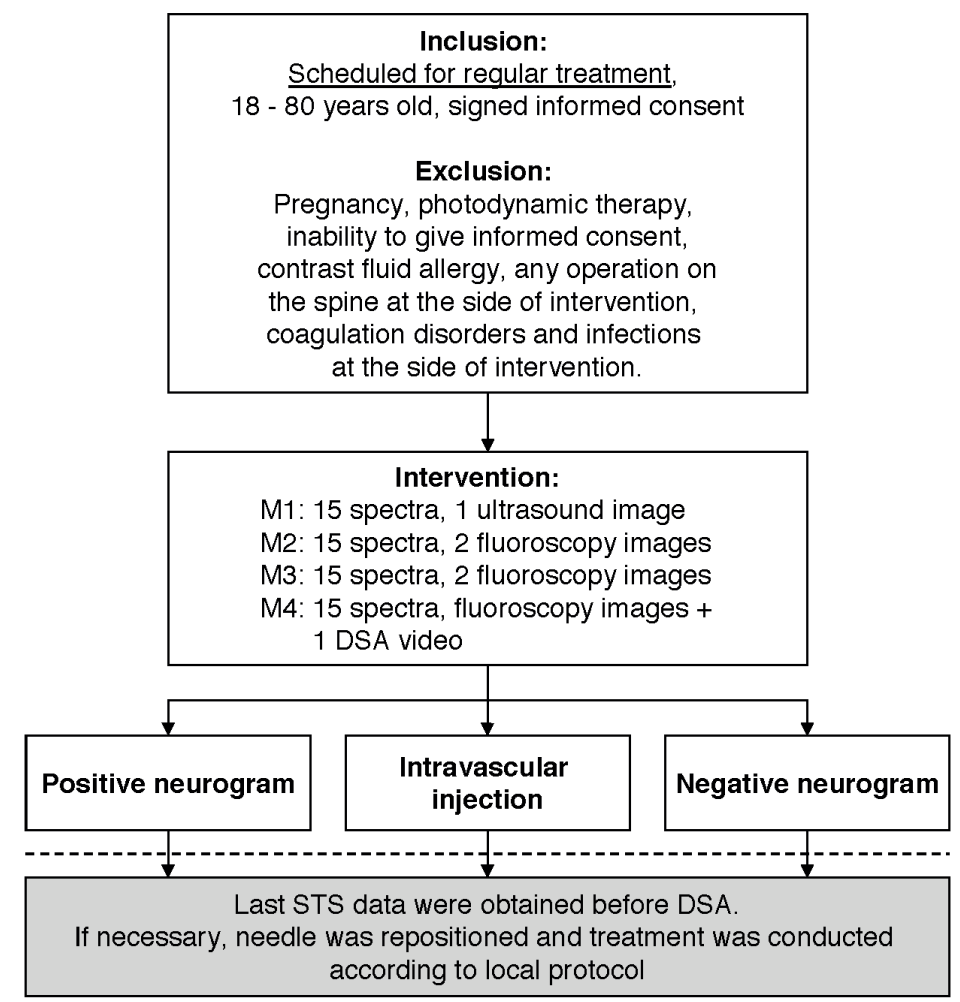

Figure 6.1 Treatment algorithm with inclusion and exclusion criteria. After the dotted line, the study ends, and the intended treatment is performed, or the needle is repositioned, and the treatment is completed if possible.

Spectral tissue sensing data were collected at 4 clearly defined needle positions along the needle trajectory (Figure 6.2), measurement location (M): M1, needle in the erector spinae muscle (confirmed by ultrasound imaging); $M 2$, needle positioned extraforaminally / at the dorsal foraminal border as defined by lateral fluoroscopy; M3, needle had passed one-third of the dorsal part inside the neuroforamen; and M4, at the end of the needle trajectory in the middle of the distance along the neuroforamen in the superior part of the neuroforamen from a lateral perspective, some millimeters cranially to the nerve. After the last STS data collection at the target location, the stylet was removed. Aspiration was performed before injection of contrast fluid during DSA. The "neurograms" obtained were used to confirm that needle placement was correct. After contrast fluid injection, the study ended, thereafter patients received standard treat- 
ment according to our pain protocol. Fluoroscopic images of M2 to M4 were saved for anterior-posterior and lateral view. Spectral tissue sensing signals were acquired at these positions. The physician indicated the level of confidence in the interpretation of the ultrasound image and DSA video at measurement points M1 and M4 using a 3-point "certainty scale" (1= uncertain, 2 = fairly certain, 3 = certain).

Spectra (reflected intensity recordings vs wavelength in the 500- to $1600-\mathrm{nm}$ range) were obtained from 3 bevel orientations (0, 90, and 180 degrees) at each measurement location. All images and videos were stored for off-line analysis. Data on patient age, sex, height, weight, reason for treatment, and relevant treatment history were also recorded. Procedure inclusion and exclusion criteria are listed in Figure 6.1.

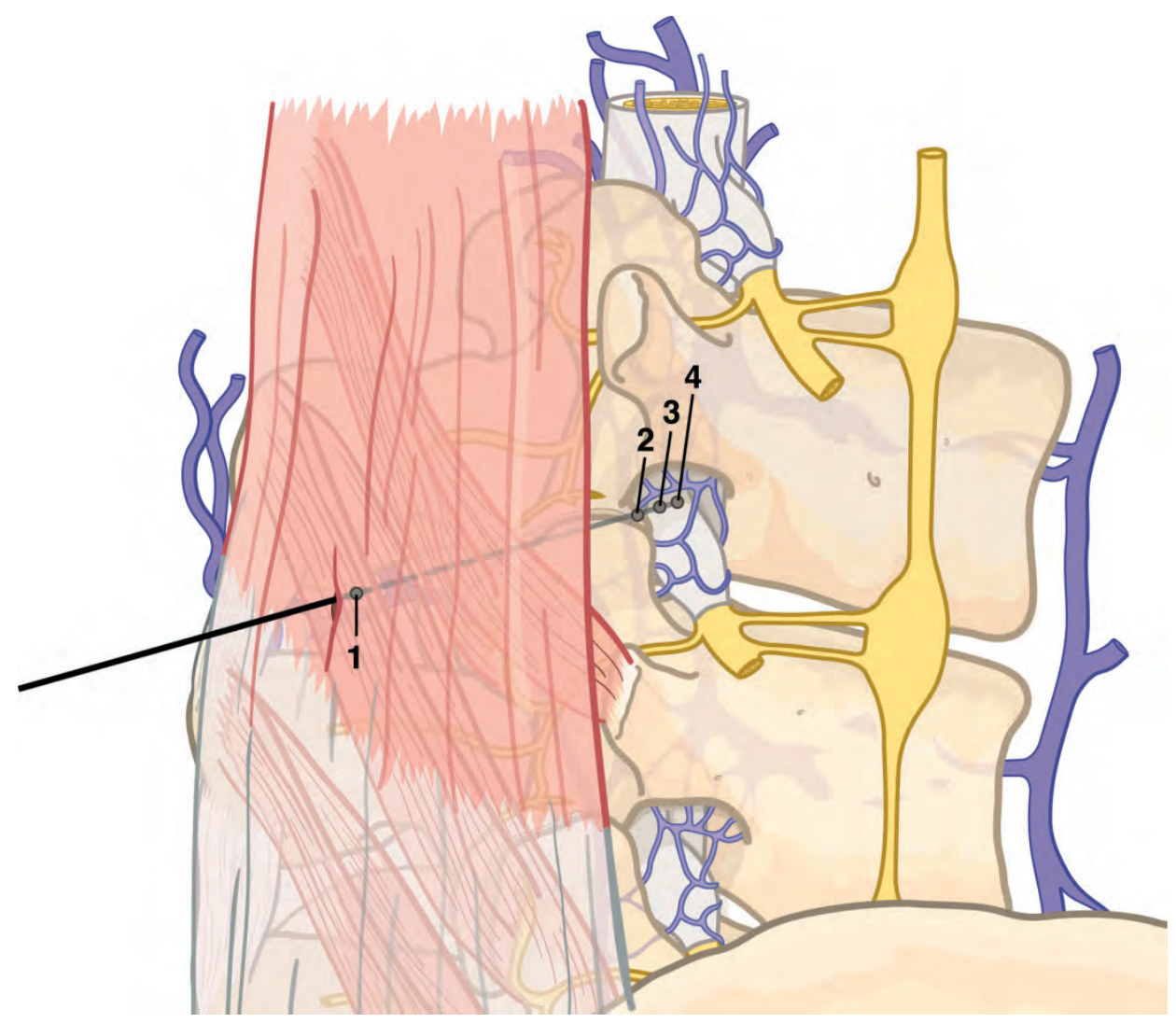

Figure 6.2 Sagittal view of the lumbar neuroforamen with the segmental nerve, surrounding fat, blood vessels, and the 4 measurement points. M1: in the paraspinal muscle, M2: at the border of the neuroforamen (intended to be extraforaminal), M3: one-third of the distance along the neuroforamen, and M4: in the middle of the neuroforamen at the target location, close to the segmental nerve. 


\section{Device description}

The STS method used in this study consists of 2 parts: an STS stylet and an optical console. The stylet was developed by Philips Research and InVivo GmbH (Schwerin, Germany) and CE marked by InVivo $\mathrm{GmbH}$. The sterile disposable stylet contains 2 fibers. The total time to signal acquisition per spectrum is 1 second. Per measurement set, 5 spectra were collected, resulting in a total acquisition time of 5 seconds per set. Spatial averages per location were obtained by recording 3 sets at different orientations per location. The sampling volume is estimated to be approximately $1 \mathrm{~mm}^{3}$. For a more detailed description of the device, see Balthasar et $\mathrm{al}^{8}$ and Nachabe et al. ${ }^{13}$

\section{Spectral analysis}

The collected spectra were wavelength calibrated, corrected for instrument response and background noise. The resulting preprocessed spectra were analyzed off-line with custom software written in Matlab (The MathWorks, Natick, Massachusetts).

Two different analysis methods were used. First, a fit model based on the work by Farrell et al ${ }^{12}$ was used to determine physiological parameters such as hemoglobin and lipid content. ${ }^{12,13}$ Second, a method based on partial least-squares discriminant analysis (PLS-DA) was used to classify needle-tip locations. ${ }^{14}$ For PLS-DA, the collected data were randomly split into either "training data sets," used to train the PLS-DA model (70\% of the data), or "validation data sets," used to evaluate the resulting model (30\% of the data). Evaluation took place by applying the PLS-DA model to the validation data and comparing the needle-tip locations predicted by the model with the locations determined by DSA. Discriminant analysis of the PLS-DA was subsequently used to obtain thresholds for discriminating between the values of the response variable.

\section{Study design}

This study was a single-blind prospective observational study in a limited number of patients undergoing an LTEI. The physician performing the LTEI was blinded for the data collected by the optical system. The physician decided at which locations STS data were collected, based on information from imaging.

An independent radiologist (C.A.) reviewed the DSA videos. He was blind to the physician-reported results and STS data. Finally, STS data were analyzed, and the data sets were compared.

\section{Primary objective}

The primary objective of the study was to assess the sensitivity and specificity of STS to discriminate between correct (safe) and incorrect placement of the needle tip during the LTEI procedure, as confirmed by contrast-enhanced fluoroscopy with DSA. Injec- 
tions in which there was spread of contrast fluid along the segmental nerve during DSA without vascular uptake were defined as "correct and safe." Incorrect needle placement was defined as extraforaminal spread or intravascular spread of contrast fluid.

\section{Secondary objective}

The secondary objective was to examine the differences between STS signals obtained at 4 predefined positions along the needle trajectory during the LTEI procedures.

\section{Sample size calculation}

The main goal of this study was to establish the capability of the STS system to determine whether the needle is correctly placed. We aimed at determining the sensitivity of the STS system with a precision of $10 \%$. Because a sensitivity to detect intravascular needle placement of real-time fluoroscopy of 71\% compared with DSA has been reported, we expected a sensitivity of $70 \%$. To reach the selected precision, 21 patients were needed in which DSA showed the correct placement. Based on an article by Furman et al., ${ }^{15}$ we assumed that in $75 \%$ of the total included patients the needle would be placed correctly on the first attempt. This meant that 28 patients were needed in total. Two more patients were included to correct for any potential technical failure during the procedures.

\section{Statistical analyses}

The differences in the STS data on hemoglobin and lipid at positions M1 and M4 were analyzed using a nonparametric Kruskal-Wallis test (2-tailed). The criterion for statistical significance was $P<0.05$. Post hoc tests on the Kruskal-Wallis test were performed using a conservative Bonferroni correction to adjust for multiple pairwise comparisons.

\section{Results}

\section{Study population}

All patients were recruited from the pain clinic at the Maastricht University Medical Center, provided written informed consent to participate, and met the inclusion criteria for participation (Figure 6.1). We collected data from 30 patients (21 women; 9 men) during a period of 5 months (November 2011 to end of March 2012). The youngest patient was 38 years old, and the oldest was 79 years (mean age, 56.63 years). Patient body mass index varied from 20 to $40 \mathrm{~kg} / \mathrm{m}^{2}$ (mean, $28 \mathrm{~kg} / \mathrm{m}^{2}$ ). 
The injection site was close to nerve root L3 in 4 cases, L4 in 9 cases, and L5 in 17 cases. The reasons for injection were radicular pain due to disc herniation (15 cases), lateral recess stenosis (11 cases), and/or radiculopathy with no detectable compression on scan but with clinical symptoms (4 cases).

\section{Procedure}

In all but 1 patient, data collection was completed as intended. A positive neurogram and a negative DSA were achieved at the first attempt in 23 (77\%) of 30 cases. In 1 of the 7 cases in which the first injection attempt failed (patient 16), an intravascular needle position was detected with DSA; in the other 6 cases, the neurogram was negative. In 1 of 30 cases (patient 2), DSA was not available during the injection procedure for technical reasons, so live fluoroscopy was used instead. The fluoroscopic images were observed during the procedure by 3 experienced physicians, who judged that needle placement was "correct" as the neurogram was positive and vascular uptake was excluded; data from this patient were therefore included in the data analysis.

The certainty scores relating to intramuscular position (M1) were "certain" (3) in all cases. Certainty scores for the final needle position (M4) and observed contrast spread were "uncertain" for 2 cases (patients 7 and 11). The M4 data from these 2 patients were excluded. In all cases, aspiration was negative. The DSA for patient 16 clearly indicated vascular uptake; the radiologist confirmed this independently. In the case of patient 21, venous blood filled the needle when the stylet was removed; nevertheless, the subsequent DSA was negative. There was $100 \%$ agreement between the observations made by the physician and the radiologist.

\section{Results from STS}

The STS data were collected from all patients, yielding 1800 spectra. Sets of spectra collected at the same measurement location were averaged to give 360 independent averaged spectra.

Figure 6.3 shows an example of the imaging data and associated optical spectra. To investigate differences between the spectra, total hemoglobin and lipid contents were calculated using the fit method. A hemoglobin content of 1.0 was equivalent to the hemoglobin content of full blood, which on average has a hemoglobin content of 15 $\mathrm{g} / \mathrm{dL}$. The lipid volume fraction (lipid/ (lipid + water)) was calculated; a lipid content of 1.0 indicated the presence of lipid with a density of $0.86 \mathrm{~g} / \mathrm{mL}$.

Figure 6.4 presents box plots of calculated hemoglobin and lipid values for 3 different M4 situations. The box plots show that the hemoglobin content was highest in the cases where the DSA was positive, that is, intravascular needle-tip position, $\mathrm{P}=0.0061$. The box plots of the lipid content show that the calculated lipid content was lowest when the DSA was positive. There was a difference $(P=0.0078)$ between the lipid con- 
tent of muscle (M1) and the lipid content at other measurement locations. The medians and whiskers in the box plots in Figures 5.4A and 5.4B are indicative of the variances related to variations between measurements for different bevel orientations and variations between patients.
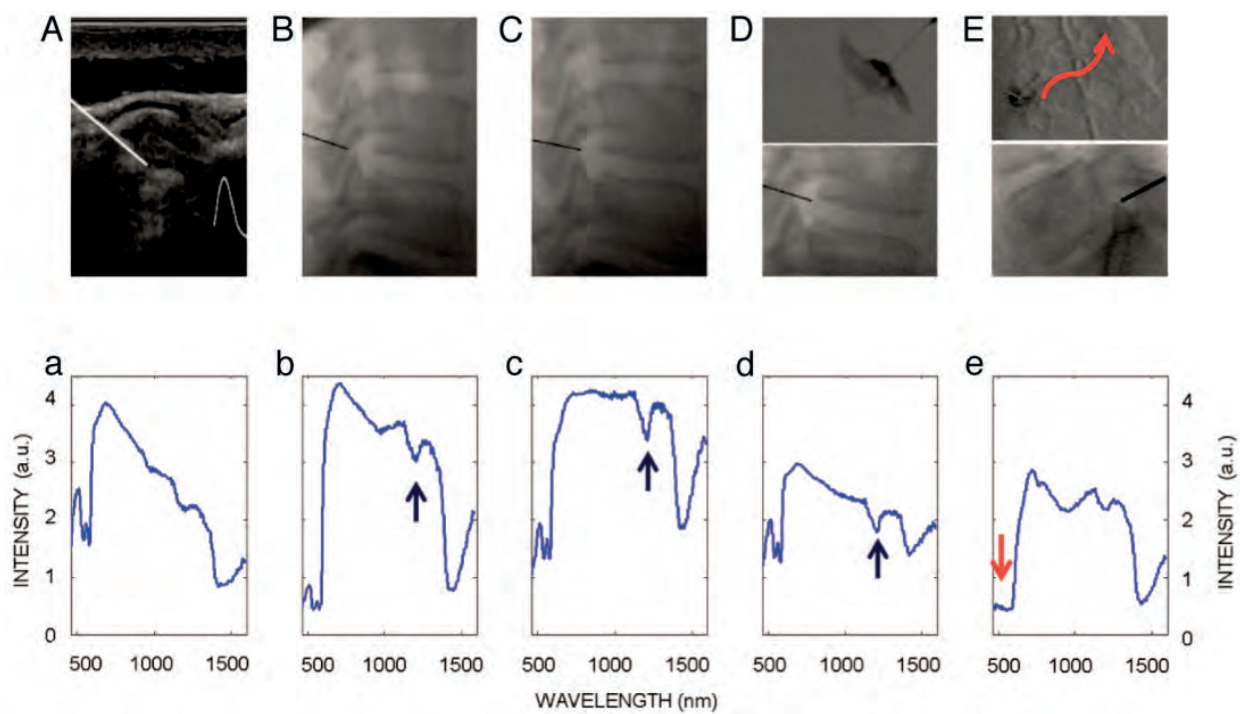

Figure 6.3 Data collected for patient $16(A, a$ and $E, e)$ and patient $9(B, b ; C, c ; D, d)$; the upper row shows images, and the corresponding optical spectra are in the lower row. A, Ultrasound image with the needle in left $\mathrm{m}$. erector spinae, (a) corresponding muscle spectrum. B, Lateral radiographic image with the needle at the border of the foramen, (b) corresponding optical spectrum. $C$, Lateral radiographic image with the needle at one-third of the foramen, (c) corresponding spectrum. D, Neurogram during DSA and lateral radiographic image with the needle in the middle of the foramen, (d) corresponding spectrum. E, Positive DSA (red arrow indicating the direction of the intravascular flow of the contrast fluid) and a lateral radiographic image with the needle in the middle of the foramen, (e) the corresponding spectrum. The black arrows in b, c, and $d$ indicate the absorption of light approximately $1200 \mathrm{~nm}$ by lipids; the red arrow in e indicates the strong absorption of light approximately $500 \mathrm{~nm}$ by hemoglobin.

Each spectrum obtained from the single measurements at specific positions was entered into the PLS-DA model, and a prediction of the tissue class was given. In the current software, the spectrum is always classified as belonging to 1 of 2 classes. For each of the results presented in the different rows of Table 6.1, a different PLS-DA model was built. Table 6.1 summarizes the sensitivity and specificity with which STS discriminated between different situations encountered at the needle tip. SDs on the estimated sensitivities and specificities ranged from $10 \%$ to $20 \%$.

Partial least-squares discriminant analysis indicated that STS discriminated between correct and incorrect needle placements with a sensitivity of $57 \%$ and specificity of $82 \%$. 
Spectral tissue sensing was most sensitive, $80 \%$, in the comparison of intraforaminal and extraforaminal measurement locations; specificity was highest, 91\%, in the comparison of positive and negative neurograms without vascular uptake. Digital subtraction angiography verified intravascular needle placement was detectable in the STS data.

\section{A Hemoglobin content}

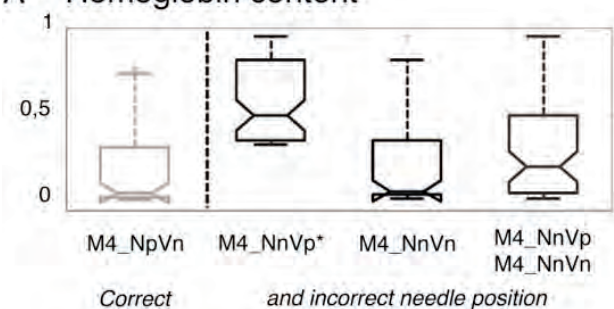

\section{B Hemoglobin content}

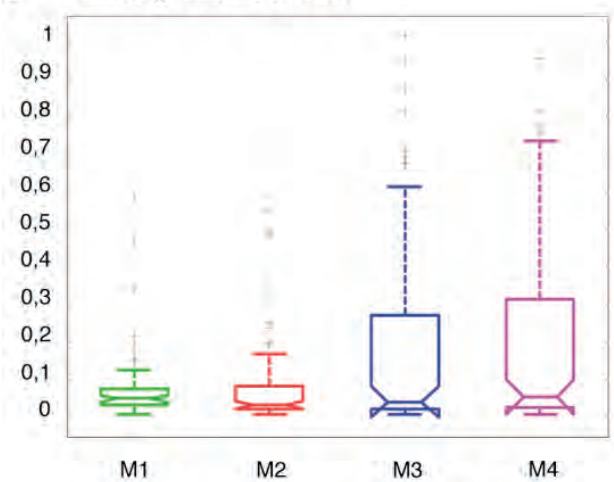

Lipid content

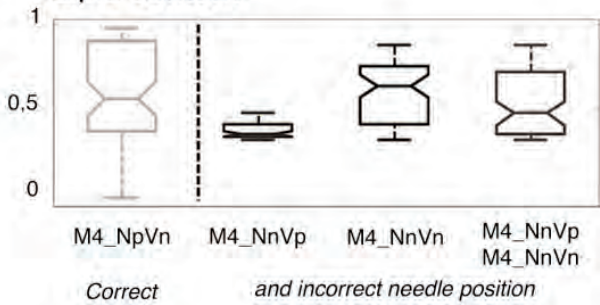

Lipid content

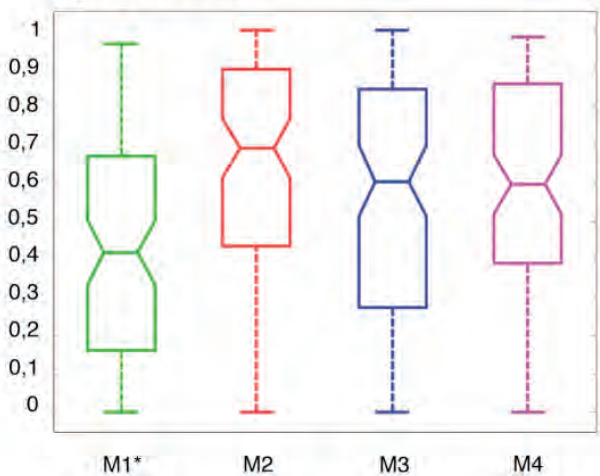

Figure 6.4 Box plots of the fit results of hemoglobin and lipid content for 4 different measurement points (M1 to M4) and different situations after DSA. A, Hemoglobin and lipid content for correct and incorrect needle placement at M4. M4NpVn: positive neurogram with no vascular uptake of contrast fluid during DSA. M4NnVp: negative neurogram, positive DSA indicating intravascular needle position. M4NnVn: negative neurogram with no vascular uptake of contrast fluid during DSA. *Significant difference $(P \leq 0.05)$. B, Hemoglobin and lipid content at all 4 measurement locations. M1: in the paraspinal muscle, M2: at the border of the neuroforamen (intended to be extraforaminal), M3: one-third of the distance along the neuroforamen, and M4: in the middle of the neuroforamen at the target location (all spectra together). *Significant difference $(P \leq 0.05)$. In all boxes, the central mark is the median; the edges of the box are the 25th and 75th percentiles. The whiskers extend to the most extreme data points not considered outliers (percentiles 0100). Outliers are plotted individually (+). 
Table 6.1 Partial least-squares discriminant analysis results for various comparisons of tissue classes, sensitivity, specificity, and number of data sets.

\begin{tabular}{|c|c|c|c|c|}
\hline Comparisons & Sensitivity & Specificity & $\mathrm{N}$ Train & N Valid \\
\hline $\begin{array}{l}\text { Correct vs all incorrect needle placement (M4_NpVn vs M4_Vp and } \\
\text { M4_NnVn) }\end{array}$ & 0.57 & 0.82 & 59 & 25 \\
\hline $\begin{array}{l}\text { Correct needle placements vs positive DSA (M4_NpVn versus } \\
\text { M4_NnVp) }\end{array}$ & 0.78 & 0.89 & 50 & 22 \\
\hline $\begin{array}{l}\text { Correct needle placements vs negative neurogram and DSA } \\
\text { (M4_NpVn vs M4_NnVn) }\end{array}$ & 0.60 & 0.91 & 54 & 24 \\
\hline Target location vs all other locations (M4 versus M1, M2 and M3) & 0.70 & 0.64 & 235 & 101 \\
\hline Extraforaminal vs intraforaminal ( $\mathrm{M} 3$ and $\mathrm{M} 4 \mathrm{vs.M} 1$ and $\mathrm{M} 2$ ) & 0.80 & 0.71 & 236 & 100 \\
\hline
\end{tabular}

$\mathrm{Np}$ indicates neurogram positive; $\mathrm{Nn}$, neurogram negative; $\mathrm{Vp}$, DSA positive for vascular uptake of contrast fluid; Vn, DSA negative; $N$ train, number of data sets used as the training set; $N$ valid, number of data sets used as the validation set.

\section{Discussion}

The primary objective of this study was to investigate the sensitivity and specificity of STS as a tool for discriminating between correct and incorrect intraforaminal placement of the needle tip during LTEls. The fit results (Figure 6.4A) showed that data on the hemoglobin and lipid content did not discriminate between correct and incorrect needle placements with statistical significance. Partial least-squares discriminant analysis showed that STS discriminated between correct and incorrect needle placement with a sensitivity of $57 \%$ and a specificity of $82 \%$. Studies of peripheral nerve stimulation in locoregional anesthesia showed a sensitivity of $74.5 \%$ of the nerve stimulator to identify needle-nerve closeness. ${ }^{16}$

Intravascular needle placement was judged as incorrect. The highest values for hemoglobin content were associated with positive DSA. High calculated hemoglobin content values were, however, also obtained in other cases, indicating that, in some instances, STS data suggest a blood-rich injection environment. Digital subtraction angiography was performed after the completion of STS data acquisition, in accordance with standard clinical practice and the study protocol. Therefore, it was not possible to investigate whether needle placement had been intravascular at an earlier stage. In 1 case, venous blood infiltrated the needle spontaneously during the retraction of the stylet. Spectral tissue sensing data from this patient indicated a high hemoglobin content at M4 (average, 0.88). The clinical relevance of the high hemoglobin content in the absence of positive DSA is unclear. It is possible that STS gives high readings for the hemoglobin content in the context of intraforaminal hematoma. However, these findings might also indicate that DSA, the strongly recommended technique for detecting intravascular needle placement, is insufficiently precise. ${ }^{17}$ There have been reports of neurological complications caused by the potential vascular uptake of medication in cases where DSA was used. ${ }^{18}$ The discrepancy between the STS and DSA data might also 
be related to bone contact. The periosteum, as a mediator of osseous vascularization, is a blood-rich environment. ${ }^{19}$ In this study, bone contact was detected 3 times by the physician during progression of the needle from M2 to M3. Nevertheless, STS is the first method with a validated ability to identify correct needle placement at the intraforaminal level in humans during LTEI.

The secondary objectives of the study were to examine the differences between STS signals obtained at 4 different measurement locations. Our data indicate that M1 can be discriminated from M2, M3, and M4 by the significantly lower lipid content $(P=0.0078)$. An earlier study on STS reported that intramuscular needle placement was associated with a higher hemoglobin and lower lipid content, ${ }^{9}$ but in the current study, we were able to replicate only the finding of a lower lipid content at intramuscular needle-tip locations.

Partial least-squares discriminant analysis showed that STS is capable of discriminating M4 from M1, M2, and M3 with a sensitivity of $70 \%$ and a specificity of $64 \%$. Sensitivity and specificity increased to $80 \%$ and $71 \%$, respectively, if the comparison was between all intraforaminal locations $(M 3+M 4)$ and all extraforaminal locations (M1 + $M 2$ ). These results suggest that STS can detect the difference between an intraforaminal and an extraforaminal needle position. This is the first study to provide information on STS and its potential during LTEIs. In our opinion, the sensitivity of $57 \%$ of STS to identify a correct needle placement is too low, and further development of the technique is necessary to improve these numbers.

There are 2 main limitations to this study. The first is the observational setup. The analysis would have been more powerful if we had been able to include an additional group in which DSA was performed whenever STS indicated a high hemoglobin content. Second, the needle-tip positions at M2 and M3 were defined on the basis of lateral radiographic images, which is not a very precise technique; computed tomographyguided procedures with 3-dimensional reconstruction of the needle-tip location would have provided more accurate location data at the cost of high radiation exposure.

Reports of investigations into serious adverse events and complications after LTEI have called for more precise needle-tip positioning. ${ }^{4}$ This is particularly true for the cervical area. ${ }^{3}$ The current imaging techniques and orientation aids have limitations; this was the rationale for trying the STS method and exploring its value during pain intervention procedures. Besides its discriminative capacity, the addition of STS to standard imaging procedures has some practical advantages: the light used for measurements is not harmful to the patient, no contrast agent needs to be injected to confirm intravascular needle placement, and results are observer-independent. Use of STS does not change the logistics of the injection procedure and would not significantly prolong it. At this stage of development of STS, we can only recommend the combined use of STS with fluoroscopy, DSA, or ultrasound.

Further research is needed to evaluate whether STS is suitable to replace contrast fluid injection and to explore if STS is able to differentiate particularly nerve tissue from 
surrounding tissue. The impact of the STS method on patient safety is unknown. Generally speaking, it is very difficult to demonstrate the absolute value and improvement in safety because of the low incidence of complications during LTEIs. ${ }^{20}$ Nevertheless, STS is a promising new technique and requires further development.

In conclusion, in the context of LTEI, the sensitivity (78\%) of STS (based on 1 case of positive DSA) in this study to detect intravascular needle position in comparison with DSA is the highest reported in the literature. This result corroborates our earlier research. ${ }^{8}$ Future research should focus on the identification of nerves and nerve fascicles using STS. 


\section{References}

1. Hong JH, Huh B, Shin $\mathrm{HH}$. Comparison between digital subtraction angiography and realtime fluoroscopy to detect intravascular injection during lumbar transforaminal epidural injections. Reg Anesth Pain Med. 2014;39:329-32.

2. Kim do W, Han KR, Kim C, Chae YJ. Intravascular flow patterns in transforaminal epidural injections: a comparative study of the cervical and lumbar vertebral segments. Anesth Analg. 2009;109:233-9.

3. Manchikanti L, Malla Y, Wargo BW, Cash KA, Pampati V, Fellows B. A prospective evaluation of complications of 10,000 fluoroscopically directed epidural injections. Pain Physician. 2012;15:131-40.

4. Huntoon MA, Martin DP. Paralysis after transforaminal epidural injection and previous spinal surgery. Reg Anesth Pain Med. 2004;29:494-5.

5. Bashkatov AN, Genina EA, Kochubey VI, Tuchin VV. Optical properties of human skin, subcutaneous and mucous tissues in the wavelength range from 400-2000 nm. J Phys D Appl Phys. 2005;38:2543-55.

6. Schenkman KA, Marble DR, Feigl EO, Burns DH. Near infrared spectroscopic measurement of myoglobin oxygen saturation in the presence of hemoglobin using partial least-squares analysis. Appl Spectrosc. 1999;53:325-31.

7. Brynolf M, Sommer M, Desjardins AE, et al. Optical detection of the brachial plexus for peripheral nerve blocks: an in vivo swine study. Reg Anesth Pain Med. 2011;36:350-7.

8. Balthasar A, Desjardins AE, van der Voort $M$, et al. Optical detection of vascular penetration during nerve blocks: an in vivo human study. Reg Anesth Pain Med. 2012;37:3-7.

9. Balthasar $A$, Desjardins $A E$, van der Voort $M$, et al. Optical detection of peripheral nerves: an in vivo human study. Reg Anesth Pain Med. 2012;37:277-82.

10. Rathmell JP, Desjardins AE, van der Voort M, et al. Identification of the epidural space with optical spectroscopy: an in vivo swine study. Anesthesiology. 2010;113:1406-18.

11. van Boxem K, van Bilsen J, de Meij N, et al. Pulsed radiofrequency treatment adjacent to the lumbar dorsal root ganglion for the management of lumbosacral radicular syndrome: a clinical audit. Pain Med. 2011;12: 1322-30.

12. Farrell TJ, Patterson MS, Wilson B. A diffusion theory model of spatially resolved, steady-state diffuse reflectance for the noninvasive determination of tissue optical properties in vivo. Med Phys. 1992;19: 879-88.

13. Nachabe R, Evers DJ, Hendriks BH, et al. Diagnosis of breast cancer using diffuse optical spectroscopy from 500 to $1600 \mathrm{~nm}$ : comparison of classification methods. J Biomed Opt. 2011;16:087010.

14. Hendriks BHWBA, Balthasar AJR, Lucassen GW, et al. Nerve detection with optical spectroscopy for regional anesthesia procedures. J Transl Med. 2015;13:380.

15. Furman MB, Lee TS, Mehta A, Simon JI, Cano WG. Contrast flow selectivity during transforaminal lumbosacral epidural steroid injections. Pain Physician. 2008;11:855-61.

16. Perlas A, Niazi A, McCartney C, Chan V, Xu D, Abbas S. The sensitivity of motor response to nerve stimulation and paresthesia for nerve localization as evaluated by ultrasound. Reg Anesth Pain Med. 2006;31:445-50.

17. El Abd OH, Amadera JE, Pimentel DC, Pimentel TS. Intravascular flow detection during transforaminal epidural injections: a prospective assessment. Pain Physician. 2014;17:21-7.

18. Chien GC, Candido KD. Digital subtraction angiography is not the answer for safe epidural injections. Pain Physician. 2014;17:E413-4

19. Simpson AH. The blood supply of the periosteum. J Anat. 1985;140:69-704.

20. Jasper JF. Role of digital subtraction fluoroscopic imaging in detecting intravascular injections. Pain Physician. 2003;6:369-72. 





\subsection{Summary}

The aim of this thesis was to determine the value of spectral tissue sensing in percutaneous needle-based procedures. In the introduction the history of needle-based procedures and safety aspects in locoregional anesthesia and pain medicine are described. Also, current maneuvers and tools are discussed with regard to their shortcomings, sensitivity, and specificity. The introduction ends with a brief description of light as an imaging tool and the Spectral tissue sensing (STS) system.

The first chapter explains the ideal method of analyzing optical data that are obtained by STS. The according research question is:

1. What is the best way to analyze optical spectra with the STS system?

The second chapter centers on the discovery of intravascular needle position. The according research question is:

2. Is it possible to detect intravascular needle position with the STS system?

The third chapter emphasizes the potential of STS to detect nerve structures. The according research question is:

3. What is the optical signature of nerve tissue?

The fourth chapter focuses on the detection of tissue transition by STS. The according research question is:

4. Is it possible to detect tissue transitions with the STS system during peripheral nerve blocks?

The fifth chapter concentrates on the identification of a correct and safe injection environment during lumbar transforaminal epidural injections with the use of STS. The according research question is:

5. Can the most appropriate and the safest injection environment be determined with the STS system during lumbar transforaminal epidural injections?

\subsection{Findings}

\subsubsection{Ad 1: What is the best way to analyze optical spectra with the STS system?}

Spectral tissue sensing STS, based on diffuse reflectance spectroscopy (DRS), belongs to the field of biomedical optics. The optical characteristics of various tissues are well de- 
scribed. Several challenges must be addressed in developing a technology that is based on DRS-i.e., how to extract the relevant optical data from the spectrum and how to analyze and translate the acquired spectral data into clinically relevant parameters.

In a previous in vivo study, we performed partial least-squares discriminant analysis (PLS-DA). ${ }^{1}$ PLS-DA was used to classify needle tip locations, and the data were split randomly into training datasets, used to train the PLS-DA model (70\% of the data), or validation datasets, used to evaluate the resulting model (the remaining 30\%). The evaluation was conducted by applying the PLS-DA model to the validation data and comparing the needle tip locations that it predicted using the locations that were determined by fluoroscopy and DSA. Discriminant analysis was subsequently performed to obtain thresholds for differentiating between values of the response variable. Using this method, the sensitivity and specificity of a technology that is used during lumbar transforaminal epidural injections were described for the first time.

In a subsequent setting, we compared 3 classification methods-PLS-DA, support vector machine (SVM), and classification and regression trees (CARTs)-which were used to examine the difference between ex vivo human fascicular and nonfascicular nerve tissue. SVM performed best on this task. The sensitivity and specificity of SVM were comparable or slightly higher compared with PLS-DA (Table 7.1). SVM performed well on multidimensional parameter spaces, because it can ignore features that do not significantly contribute to the discrimination. Dimension reduction by fitting allowed to exclude parameters that are different in an in vivo or ex vivo setting such as blood oxygenation. In general, the 3 methods had similar accuracies.

Table 7.1 Classification results according to SVM, PLS-DA and CART for discrimination of fascicular tissue of the nerve from the surrounding tissues. For SVM different feature selection methods are used: fit parameters, PCA, segments and a combination of the last three. For PLSDA, 10 principal components have been used (10PC's). For the CART analysis, the fit parameters have been used as features. Matthews correlation coefficient (MCC see Eq. 2, Chapter 2), accuracy (ACC) sensitivity (SENS), specificity (SPEC).

\begin{tabular}{|c|c|c|c|c|c|}
\hline Classification method & Feature selection & MCC & $\mathrm{ACC}$ & SENS (\%) & SPEC (\%) \\
\hline SVM & Fit & 0.711 & 0.854 & 82.6 & 88.8 \\
\hline SVM & PCA & 0.793 & 0.897 & 89.9 & 89.5 \\
\hline SVM & Segments & 0.779 & 0.890 & 88.6 & 89.5 \\
\hline SVM & Combined & 0.826 & 0.914 & 91.3 & 91.4 \\
\hline PLSDA & $10 P C^{\prime} s$ & 0.814 & 0.907 & 92.5 & 89.5 \\
\hline CART & Fit & 0.615 & 0.808 & 81.2 & 80.4 \\
\hline
\end{tabular}




\subsubsection{Ad 2: Is it possible to detect intravascular needle positions by STS?}

During locoregional anesthesia and interventional pain medicine, an intravascular needle position should be avoided. For instance, intravascular injection of local anesthetics can lead to severe complications. Several approaches were used to address this research question in 3 in vivo settings. The first setting was in patients for whom interventional pain procedures were planned. In 2 cases, an unintended intravascular needle position was detected by live fluoroscopy ${ }^{2}$-these 2 situations were clearly detected by STS.

The second observational study was also performed in interventional pain procedures. An intravascular needle position was detected in 1 case by DSA. In a second case, the needle was passively infiltrated by venous blood. ${ }^{1}$ Both cases were identified with STS, as evidenced by high blood content. In this study, other needle positions with greater blood content were detected by STS. Due to the study design in this situation, no DSA or aspiration was performed, and the impact of high blood content measured by the STS in this situation is unknown.

Due to the low number of cases in the first 2 studies, we decided to perform a randomized, single-blind, controlled trial with planned intravascular needle insertion, ${ }^{3}$ in which we inserted a needle subcutaneously in 20 volunteers, who were then randomized for a second subcutaneous or intravascular insertion. The needle position was controlled by aspiration and ultrasound. Based on our results, STS detected an intravascular needle position with high significance. In this setting, the STS data corresponded $100 \%$ with the aspiration, compared with $75 \%$ between STS and US.

For the latter, in the remaining $25 \%$ of cases, the anesthesiologist assumed an intravenous needle position, based on the US image. In these cases, during the insertion, the needle tip positions were judged to be intravenous, but on removal of the stylet, there was no aspiration of blood. The spectra for these subjects suggested a subcutaneous needle position.

Thus, intravascular needle tip positions can't be completely excluded with this study design. Based on aspiration of blood, intravascular needle positioning might fail to be detected. Injection of saline or contrast fluid might have helped ensure the needle position, neither of which was used in this study.

\subsubsection{Ad 3: What is the optical signature of nerve tissue?}

In addition to the detection of blood vessels, clear identification of nerve tissue is paramount for an anesthesiologist. Despite the use of ultrasound or nerve stimulation, cases of nerve damage are still reported. ${ }^{4-6}$ The incidence of complications after locoregional anesthesia is $0.4 \%$ to $0.79 \%{ }^{7,8}$ However, patients and clinicians fear neurological complications due to nerve damage, such as leg weakness and neuropathic pain. ${ }^{9,10}$ Even temporary nerve damage has a major impact on quality of life. ${ }^{11}$ 
We examined nerve detection in vivo during ultrasound-guided nerve blocks. In this in vivo setup, we failed to discriminate nerve tissue from other adipose tissue, ${ }^{12}$ prompting us to revert back to ex vivo studies. DRS was acquired from 156 locations in 19 human cadavers in the wavelength range of 400-1710 nm. ${ }^{13}$ Fascicular nerve tissue, muscle, sliding fat, and subcutaneous fat were compared. Fascicular nerve tissue was discriminated from other tissues with a sensitivity and specificity of approximately $90 \%$.

Based on this finding, we decided to perform a second ex vivo study to determine the morphological, biological, chemical, and optical characteristics of human nerve tissue and adipose tissue. In addition to conducting DRS and measuring absorption, we performed histology and high-resolution magic-angle spinning nuclear magnetic resonance (HR-MAS NMR) spectroscopy and 2D ${ }^{13} \mathrm{C}-{ }^{1} \mathrm{H}$ heteronuclear single-quantum coherence (HSQC). As a result, DRS was able to differentiate nerve fascicles from other adipose tissue. According to the optical absorption measurements in the fascicular nerve tissue, we observed different absorptions of light and a shift in the peak between $900 \mathrm{~nm}$ and $1000 \mathrm{~nm}$. With regard to the DRS and HR-MAS NMR measurements, the water percentage of nerve fascicles was higher and the fat percentage was lower compared with all other tissues. The difference in collagen in the DRS measurement was greater in fascicular nerve tissue, as supported by histology. The high scattering parameter (b) observed by DRS in fascicular nerve tissue may be related to a larger inhomogeneity in the distribution of the collagen content and higher density of cellular nuclei as seen in histology. Comparing the results between this study and the earlier trial, the differences in collagen, water, light scattering, and fat in fascicular nerve tissue were reproduced.

\subsubsection{Ad 4: Is it possible to detect tissue transitions with the STS system during peripheral nerve blocks?}

During percutaneous peripheral nerve blocks, the needle will pass various tissues en route to the target nerve. Generally, subcutaneous adipose tissue and muscles will be punctured by the needle before the nerve region is reached. To determine whether STS detects this tissue transition, we made 20 measurements during ultrasound-guided nerve blocks ${ }^{12} ; 15$ datasets were ultimately included in the analysis. The lipid parameter declined significantly and the hemoglobin parameter rose significantly when the needle tip transited from subcutaneous fat to muscle tissue. When the needle passed from muscle tissue to the nerve environment, the lipid parameter increased significantly, and the hemoglobin parameter fell again. Discrimination between subcutaneous fat and nerve environment was not possible at this time with these parameters.

In a second study, we examined the differences between STS signals obtained at 4 different measurement locations during transforaminal epidural injection (TEI). The 4 measurement points were: M1: in the paraspinal muscle, M2: at the border of the neuroforamen (intended to be extraforaminal), M3: one-third of the distance along the neuroforamen, and M4: in the middle of the neuroforamen at the target location, close 
to the segmental nerve. Our data indicated that $\mathrm{M} 1$ can be discriminated from $\mathrm{M} 2, \mathrm{M} 3$, and M4 by the significantly lower lipid content $(P=0.0078)$. An earlier study on STS reported that intramuscular needle placement was associated with a higher hemoglobin and lower lipid content, but in the current study, we were able to replicate only the finding of a lower lipid content. The comparison of all intraforaminal locations (M3 + M4) and all extraforaminal locations (M1 + M2) showed a sensitivity and specificity increased to $80 \%$ and $71 \%$, respectively. These results suggest that STS can detect the needle transition from extraforaminal to an intraforaminal needle position.

\subsubsection{Ad 5: Can the most appropriate and safest injection environment be determined with the STS system during lumbar transforaminal epidural injections?}

Transforaminal epidural injection (TEI) is one of the most frequently performed procedures in interventional pain medicine. Nevertheless, case reports have described severe complications, such as spinal cord or brain infarction. Reports on serious adverse events and complications after TEI have recommended more precise needle tip positioning, ${ }^{14,15}$ particularly for the cervical area. ${ }^{16}$ Current imaging techniques and orientation aids have limitations, and undetected intravascular uptake of injection fluid can cause such severe complications. ${ }^{17}$

We tested STS in lumbar TEls. ${ }^{1}$ A safe injection environment was defined as the spread of contrast fluid along the segmental nerve during DSA without vascular uptake. In this study, with regard to correct needle placement the specificity of STS was $82 \%$, and its sensitivity was $57 \%$. Intraforaminal versus extraforaminal locations were identified with a sensitivity of $80 \%$ and a specificity of $71 \%$. In the literature, no comparable studies have been performed that describe the sensitivity or specificity of correct needle placement in lumbar TEls with other imaging techniques.

\subsection{General discussion}

This thesis, Eyes of the needle - Spectral tissue sensing, an innovative technology to detect different types of tissues during needle-based procedures in locoregional anesthesia and pain medicine, examines the potential of biomedical optics in anesthesiology practice. The basic principle that underlies all optical devices for diagnostic or therapeutic purposes is that the behavior of light of certain wavelengths in various situations is analyzed. Researchers have exploited this phenomenon to develop many devices to characterize tissues, analyze the composition of gases, and destroy tissues. In today's anesthesiology practices, light is used as a diagnostic tool for pulse oximetry and the analysis of the anesthetic gas, for example. 
The concept of measuring $\mathrm{O} 2$ saturation with light was first described in 1935 by German physician Karl Matthes (1905-1962). ${ }^{18}$ Also, in surgery, several optical technologies are used to discriminate various types of tissue. Fluorescence-guided surgery, using fluorescein sodium, was introduced by Moore et al. in $1948 .{ }^{19}$ Near-infrared fluorescence is used in cancer surgery to determine which tissues must be resected or avoided. $^{20}$

Raman analysis is a well-known technique in biomedical optics that is based on the inelastic scattering of photons on interaction with tissue, measuring the vibrational fingerprint of cells and tissues. ${ }^{19}$ For instance, Raman spectra have been used to discriminate between malignant and benign tissue in breast and head and neck cancer patients. $^{21,22}$

Spectroscopy is another optical technique, using optical probes to determine optical tissue properties to determine tissue composition (based on the absorption characteristics of various chromophores) and tissue architecture (scattering). In contrast to general anesthesia and surgery, no optical technologies have been established in locoregional anesthesia or pain medicine.

Spectral tissue sensing (STS), examined in this thesis, is an optical technique based on diffuse reflectance spectroscopy (DRS). We developed an optical tissue database to render STS a valuable tool for percutaneous needle-based procedures in (locoregional) anesthesia and pain practice. As discussed in the introduction, current techniques have shortcomings, necessitating improvements in their safety and performance. ${ }^{4,17,23,24}$

To construct a database, we first determined the tissues are most important to be identified, as supported by the literature and ongoing discussion among experts. ${ }^{4,25,26}$ This thesis focuses on the identification of adipose tissue, muscle, blood, and nerve fascicles.

The optical characteristics of the following tissues were described:

- Adipose tissue:

o Low scattering

o High absorption of light at $1200 \mathrm{~nm}$

- Muscle tissue:

o High scattering

o Some absorption of light $500 \mathrm{~nm}$ and little absorption at $1200 \mathrm{~nm}$

- Blood:

o Low scattering

o High absorption of light between 500-600 nm

- Nerve fascicles:

o High scattering

o Absorption of light at 1300-1600, 1900-2200 nm

o Peak shift 900 and $1000 \mathrm{~nm}$ 


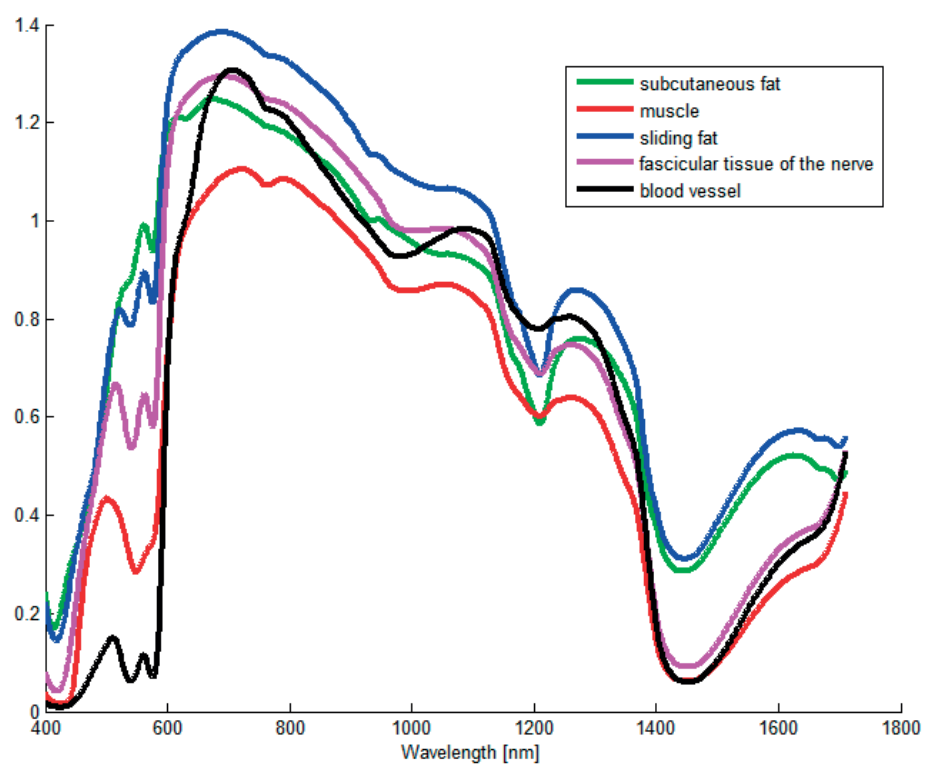

Figure 7.1 Overview of typical absorption characteristics of subcutaneous and sliding fat, muscle, blood, and nerve fascicles. X-axis wavelength in $\mathrm{nm}, \mathrm{Y}$-axis intensity in arbitrary units.

With regard to intravascular needle position, nerve stimulators have been unable to identify blood vessels, and aspiration has been needed to test for intravascular needle position. Aspiration has a sensitivity of only $25 \%$ to $47 \% .{ }^{27}$ Using X-ray, contrast fluid is needed to test for intravascular needle position. Ultrasound yields more anatomical information, but it is a complex and challenging tool with a steep learning curve. Moreover, the outcome of the procedure depends highly on the experience of and interpretation by the physician. ${ }^{4,28}$ With ultrasound or DSA, intravascular injections continue to encounter complications. ${ }^{25,29,30}$ STS could provide important information on the tissue in front of the needle and detect intravascular needle position reliably, as proven by 3 studies in this thesis.

With the use of ultrasound still unintended subepineural injections occur. ${ }^{31-33}$ Intraneural needle position does not always lead to a neurological damage one of the most feared complications. There is a clinical need to improve needle tip identification. ${ }^{5,34,35}$ Various technologies are being examined to improve needle tip placement, such as intraneural needle detection with bioimpedance monitoring. ${ }^{36}$ Further, more advanced ultrasound machines are being designed. Real-time 3-dimensional ultrasound allows for simultaneous visualization of multiple planes of view, permitting longitudinal, cross-sectional, and coronal images to be viewed without probe adjustment, and provides additional information about the spatial relationship between anatomical structures of interest compared with standard imaging. ${ }^{34,37}$ 
This thesis describes the optical characteristics of fascicular nerve tissue; such studies in surgery have been published. Schols et al. used DRS in a camera system during thyroid and parathyroid resection and carpal tunnel release surgery, testing 2 hyperspectral camera technologies (silicon and indium gallium arsenide) for automated discrimination between nerves and surrounding adipose tissue. They reported accuracies of $67 \%$ and $100 \%$, respectively. ${ }^{38}$ In near-infrared fluorescence, myelin-selective fluorescent dyes and nerve-highlighting fluorescent peptides have been developed. ${ }^{39,40}$ Also, a NIR fluorescent dye for use in probes has been described. ${ }^{41}$ Many options in biomedical optics are being tested to identify nerve tissue. This thesis concentrates on the identification of nerve fascicles. The optical characteristics of ex vivo nerve fascicles are detailed. Next to the supplying blood vessels, nerve fascicles are the most vulnerable structure of the nerve. Damage to these fascicles can result in neurological symptoms, such dysesthesia and muscle weakness. STS can identify these nerve fascicles.

In addition to that of the tissue itself, information on tissue transition is helpful. These data require a fast STS system that generates user-friendly optical information in real time to the operator without disturbing the workflow. In doing so, the needle placement can be more accurate, and the needle tip identification can be simplified.

Finally, we examined whether STS can detect a safe injection environment during lumbar transforaminal epidural injections (LTEIs). ${ }^{1,12}$ The TEI is one of the most common procedures in interventional pain medicine. Case reports have described severe complications, such as spinal cord and brain infarction. ${ }^{14,15}$ In this setting, it was the first technology to have clear sensitivity and specificity with regard to needle placement during LTEI. Here, we tested the STS system in a clinical setting in actual patients and gathered information on various tissues and the tissue transition.

DRS is not a standalone technique in anesthesiology-other imaging tools, such as ultrasound and X-ray, are needed to determine the anatomical orientation and plan the procedure. STS can identify various tissues, and compared with current clinical tools, this property is unique (see also Table 7.1). The innovation of STS lies in its ability to provide the clinician during needle-based protocols with additional tissue information directly from the needle tip to complete the anatomical profile and track the needle tip position during various procedures.

\subsubsection{Validation and reliability}

A new technology must meet several criteria to render it a valuable tool. It should:

1. Consistently produce the same results, independent of the user

2. Measure what it claims to assess

This thesis contributes to the validation and reliability of STS. The STS system has clinical potential and can improve current practices in terms of safety. In various settings and patients, STS performed comparably concerning intravascular needle position. These 
results were assessed against standard tools (aspiration, live fluoroscopy, and DSA). The optical detection of adipose tissue by STS was also robust and reproduced in ex vivo and in vivo settings. Regarding the identification of fascicular nerve tissue, several important steps were developed, and optical characteristics were described in an ex vivo setting.

In general, no requirements or criteria exist to which new developed technology in locoregional anesthesia should apply. As a consequence, in this thesis, the following questions were frequently raised:

- What are the criteria for an ideal nerve localization tool for procedures in regional anesthesia or pain medicine?

- What is the niche for STS versus other imaging/guidance tools in percutaneous needle-based procedures?

As mentioned, there are no standards with regard to the needs of physicians for safe percutaneous needle-based procedures. The following section describes 14 criteria for an ideal imaging guidance tool and gives an overview of the various techniques and how well they meet each criterion.

\subsubsection{Criteria}

We have generated a list of criteria for the ideal medical device for percutaneous needlebased procedures, with which current and future techniques and tools can be classified.

These criteria were developed by an expert team at Maastricht University Medical Hospital and St. Radboud University Medical Center, Nijmegen, The Netherlands.

1. Gives a real-time view of the relevant anatomical structures in and along the planned needle trajectory

2. Reliably identifies nerve structures

3. Reliably identifies the subepineural needle position

4. Reliably identifies blood vessels

5. Reliably identifies the intravascular needle position

6. Gives a clear view of the needle tip during the procedure

7. Is primarily independent of the experience and skills of the physician

8. Is primarily independent of the observation and interpretation by the physician

9. Has a short learning curve

10. Does not cause harm to the patient or physician

11. Can be used in various patient categories (e.g. any BMI or age)

12. Does not disturb the regular workflow (no extra injections, no extra sonography, etc.)

13. Gives a real-time display of the needle tip, needle trajectory, and anatomical structures

14. Identifies tissues directly in front of the needle tip in real time during the procedure 
Table 7.1 shows the 14 criteria with regard to the main imaging tools and STS. For each imaging tool, an indication is given if it fulfills a certain criterion. The decisions were checked and confirmed by a radiologist. None of these imaging techniques or medical devices meets all of the criteria. Table 7.1 also shows the ideal combinations of various tools that fulfill the most criteria.

STS clearly scores best compared with the other tools and provides the most overlap with the other tools. Thus, STS also provides a double check of information generated by another tool. The combination of ultrasound and STS fulfills 12 of the 14 criteria. For instance, aspiration can be fully replaced by STS and nerve stimulator for most functions at this stage of development of STS. Currently, STS is unable to stimulate nerves. For Xray-based procedures, the injection of contrast fluid and DSA could be replaced by STS.

Table 7.1 Criteria for the ideal medical device during percutaneous needle-based procedures, the 4 main imaging tools (nerve stimulator, radiography, ultrasonography, and CT), and aspiration used today for these procedures. P means partly.

\begin{tabular}{|c|c|c|c|c|c|c|c|c|c|c|c|c|c|c|}
\hline & 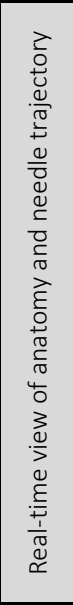 & 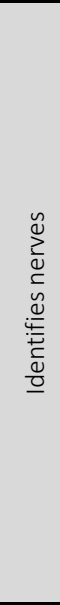 & 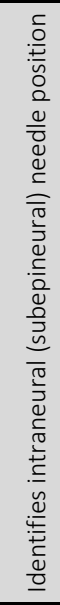 & 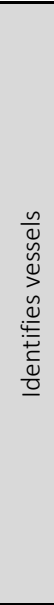 & 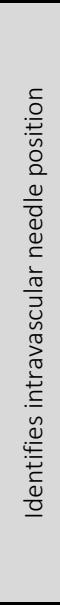 & 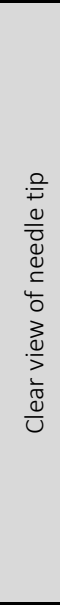 & 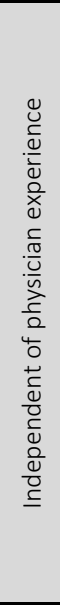 & 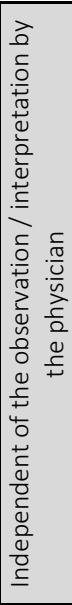 & 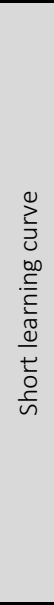 & 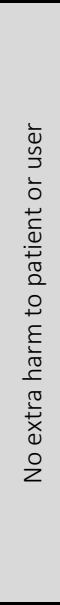 & 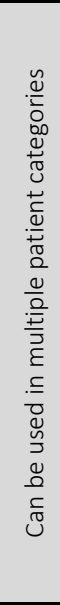 & 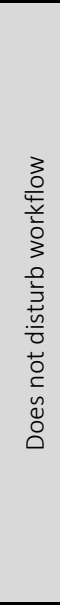 & 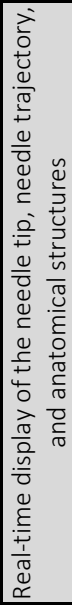 & 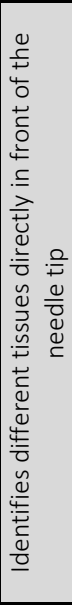 \\
\hline $\begin{array}{l}\text { Nerve } \\
\text { stimulation }\end{array}$ & No & $P^{1}$ & $P^{1}$ & No & No & No & Yes & No & Yes & $P^{3}$ & Yes & $p^{4}$ & No & No \\
\hline $\begin{array}{l}\text { Radiography } \\
\text { with DSA }\end{array}$ & No & No & No & Yes & Yes & $P^{2}$ & No & No & No & No & No & $p^{4}$ & No & No \\
\hline Ultrasound & Yes & Yes & $P^{2}$ & Yes & $P^{2}$ & $P^{2}$ & No & No & No & Yes & No & Yes & Yes & No \\
\hline $\mathrm{CT}$ & No & No & No & Yes & Yes & Yes & No & $\mathrm{P}^{2}$ & No & No & No & $P^{7}$ & No & No \\
\hline Aspiration & No & No & No & No & $P^{5}$ & No & Yes & $P^{2}$ & Yes & Yes & Yes & $P^{7}$ & No & No \\
\hline STS & No & $P^{6}$ & Yes & No & Yes & No & Yes & Yes & Yes & Yes & Yes & $p^{8}$ & No & Yes \\
\hline
\end{tabular}

${ }^{1}$ Insufficient (sensitivity of $74 \%$ ); ${ }^{2}$ dependent on physician experience; ${ }^{3}$ can be painful to the patient; ${ }^{4}$ physician needs extra help from another person or needs to inject an ancillary agent, such as contrast fluid, and take extra images; ${ }^{5}$ insufficient (sensitivity < 47\%); ${ }^{6}$ it is not clear whether STS can discover a nerve from the outside; ${ }^{7}$ extra procedures are necessary; ${ }^{8}$ depends on the design of the user interface. 


\subsubsection{Limitations}

More studies are needed in various settings to prove the robustness of existing results and to test STS in a larger, more heterogeneous group of patients.

At this time, several questions remain. In vivo research is needed to confirm the ability of STS to discriminate between nerve fascicles and other adipose tissue. The value of detecting a blood-rich environment by STS is unknown without other proof of the intravascular needle position, which can result from a hematoma or small bleed. A userfriendly interface must be developed and tested for the STS information to be easily understandable and usable.

\subsection{Future perspectives}

To develop STS into a valuable tool for standard high-quality health care, the database should be generated with all types of tissues. To this end, real-time identification of the tissue directly in front of the needle could provide important data to the physician. We see potential for STS in surgery, diagnostic punctures, needle guidance during any type of percutaneous procedure, and the documentation and quality of care. For instance, documentation of the tissues that are passed during a procedure can be stored. Tissue diagnostics by STS could be also relevant to pain practices. In cases of radiofrequency treatment, the quality of percutaneous radiofrequent lesions can be determined, and the treatment can be individualized, improving the outcome. The combination of STS with tissue-specific fluorescence has not been examined extensively.

Studies have been performed to determine the value of STS in oncology. STS can improve the differentiation of malignant versus benign tissue during surgery or biopsy. STS can be integrated into catheters or sutures to evaluate blood or tissue oxygenation. For instance, the integration of STS into intestinal sutures or central venous catheters can allow one to control tissue oxygenation or ensure venous placement, respectively.

There is some research on the optical stimulation of nerves, which might one day be performed by STS. Nerve stimulation during locoregional anesthesia and surgery has well-known functions.

Finally, an integrated user interface on the ultrasound screen could ease the workflow for the clinician, which would require a fast-acting STS system that does not disturb the workflow.

In summary, STS is a promising technique with potential value in pain and anesthesiology practices. The combination of the STS system with ultrasound or X-ray has the potential to become the new technical standard in percutaneous needle-based procedures. 


\section{References}

1. Balthasar AJ, Lucassen GW, Sommer M, van der Voort M, Gijsbers G, Arnoldussen CW, et al. The Role of Spectral Tissue Sensing During Lumbar Transforaminal Epidural Injection. Reg Anesth Pain Med. 2016;41(4):520-6.

2. Balthasar A, Desjardins AE, van der Voort M, Lucassen GW, Roggeveen S, Wang K, et al. Optical detection of vascular penetration during nerve blocks: an in vivo human study. Reg Anesth Pain Med. 2012;37(1): 3-7.

3. Balthasar AJ, van Geffen GJ, van der Voort M, Lucassen GW, Roggeveen S, Bruaset IJ, et al. Spectral tissue sensing to identify intra- and extravascular needle placement - A randomized single-blind controlled trial. PLoS One. 2017;12(3):e0172662.

4. Neal JM. Ultrasound-guided regional anesthesia and patient safety: An evidence-based analysis. Reg Anesth Pain Med. 2010;35(2 Suppl):S59-67.

5. Terkawi AS, Karakitsos D, Elbarbary M, Blaivas M, Durieux ME. Ultrasound for the anesthesiologists: present and future. ScientificWorldJournal. 2013;2013:683685.

6. Neuburger M, Schwemmer U, Volk T, Gogarten W, Kessler P, Steinfeldt T. [Localization of peripheral nerves. Success and safety with electrical nerve stimulation]. Anaesthesist. 2014;63(5):422-8.

7. Barrington MJ, Watts SA, Gledhill SR, Thomas RD, Said SA, Snyder GL, et al. Preliminary results of the Australasian Regional Anaesthesia Collaboration: a prospective audit of more than 7000 peripheral nerve and plexus blocks for neurologic and other complications. Reg Anesth Pain Med. 2009;34(6):534-41.

8. Jacob AK, Mantilla CB, Sviggum HP, Schroeder DR, Pagnano MW, Hebl JR. Perioperative nerve injury after total knee arthroplasty: regional anesthesia risk during a 20-year cohort study. Anesthesiology. 2011;114(2):311-7.

9. Barrington MJ, Snyder GL. Neurologic complications of regional anesthesia. Curr Opin Anaesthesiol. 2011;24(5):554-60.

10. Houten JK, Errico TJ. Paraplegia after lumbosacral nerve root block: report of three cases. Spine J. 2002;2(1):70-5.

11. Helen L, O'Donnell BD, Moore E. Nerve localization techniques for peripheral nerve block and possible future directions. Acta Anaesthesiol Scand. 2015;59(8):962-74.

12. Balthasar A, Desjardins AE, van der Voort M, Lucassen GW, Roggeveen S, Wang K, et al. Optical detection of peripheral nerves: an in vivo human study. Reg Anesth Pain Med. 2012;37(3):277-82.

13. Hendriks BH, Balthasar AJ, Lucassen GW, van der Voort M, Mueller M, Pully VV, et al. Nerve detection with optical spectroscopy for regional anesthesia procedures. J Transl Med. 2015;13:380

14. Manchikanti L, Malla Y, Wargo BW, Cash KA, Pampati V, Fellows B. A prospective evaluation of complications of 10,000 fluoroscopically directed epidural injections. Pain physician. 2012;15(2):131-40.

15. Huntoon MA, Martin DP. Paralysis after transforaminal epidural injection and previous spinal surgery. Reg Anesth Pain Med. 2004;29(5):494-5.

16. Van Zundert J, Huntoon MA, van Kleef M. Complications of transforaminal cervical epidural steroid injections. Spine (Phila Pa 1976). 2009;34(22):2477; author reply -8.

17. Rathmell JP. Toward improving the safety of transforaminal injection. Anesth Analg. 2009;109(1):8-10.

18. Matthes K. Untersuchungen über die Sauerstoffsättigung des menschlichen Arterienblutes. NaunynSchmiedebergs Archiv für experimentelle Pathologie und Pharmakologie.179(6):698-711.

19. Valdes PA, Roberts DW, Lu FK, Golby A. Optical technologies for intraoperative neurosurgical guidance. Neurosurg Focus. 2016;40(3):E8.

20. Vahrmeijer AL, Hutteman M, van der Vorst JR, van de Velde CJ, Frangioni JV. Image-guided cancer surgery using near-infrared fluorescence. Nat Rev Clin Oncol. 2013;10(9):507-18.

21. Hoesli RC, Orringer DA, McHugh JB, Spector ME. Coherent Raman Scattering Microscopy for Evaluation of Head and Neck Carcinoma. Otolaryngol Head Neck Surg. 2017:194599817700388. 
22. Hu C, Wang J, Zheng C, Xu S, Zhang H, Liang Y, et al. Raman spectra exploring breast tissues: Comparison of principal component analysis and support vector machine-recursive feature elimination. Med Phys. 2013;40(6Part1).

23. Rathmell JP, Manion SC. The role of image guidance in improving the safety of pain treatment. Curr Pain Headache Rep. 2012;16(1):9-18.

24. Warman P, Nicholls B. Ultrasound-guided nerve blocks: efficacy and safety. Best Pract Res Clin Anaesthesiol. 2009;23(3):313-26.

25. Bigeleisen PE. Nerve puncture and apparent intraneural injection during ultrasound-guided axillary block does not invariably result in neurologic injury. Anesthesiology. 2006;105(4):779-83.

26. Sites BD, Taenzer AH, Herrick MD, Gilloon C, Antonakakis J, Richins J, et al. Incidence of local anesthetic systemic toxicity and postoperative neurologic symptoms associated with 12,668 ultrasound-guided nerve blocks: an analysis from a prospective clinical registry. Reg Anesth Pain Med. 2012;37(5):478-82.

27. Perlas A, Niazi A, McCartney C, Chan V, Xu D, Abbas S. The sensitivity of motor response to nerve stimulation and paresthesia for nerve localization as evaluated by ultrasound. Reg Anesth Pain Med. 2006;31(5):445-50.

28. Hadzic A, Sala-Blanch X, Xu D. Ultrasound guidance may reduce but not eliminate complications of peripheral nerve blocks. Anesthesiology. 2008;108(4):557-8.

29. Sala Blanch X, Lopez AM, Carazo J, Hadzic A, Carrera A, Pomes J, et al. Intraneural injection during nerve stimulator-guided sciatic nerve block at the popliteal fossa. Br J Anaesth. 2009;102(6):855-61.

30. Robards C, Hadzic A, Somasundaram L, Iwata T, Gadsden J, Xu D, et al. Intraneural injection with lowcurrent stimulation during popliteal sciatic nerve block. Anesth Analg. 2009;109(2):673-7.

31. Chin KJ, Perlas A, Chan VW, Brull R. Needle visualization in ultrasound-guided regional anesthesia: challenges and solutions. Reg Anesth Pain Med. 2008;33(6):532-44.

32. Orebaugh SL, McFadden K, Skorupan H, Bigeleisen PE. Subepineurial injection in ultrasound-guided interscalene needle tip placement. Reg Anesth Pain Med. 2010;35(5):450-4.

33. Liu SS, YaDeau JT, Shaw PM, Wilfred S, Shetty T, Gordon M. Incidence of unintentional intraneural injection and postoperative neurological complications with ultrasound-guided interscalene and supraclavicular nerve blocks. Anaesthesia. 2011;66(3):168-74.

34. Clendenen NJ, Robards CB, Clendenen SR. A standardized method for 4D ultrasound-guided peripheral nerve blockade and catheter placement. Biomed Res Int. 2014;2014:920538.

35. Abdallah FW, Macfarlane AJ, Brull R. The Requisites of Needle-to-Nerve Proximity for Ultrasound-Guided Regional Anesthesia: A Scoping Review of the Evidence. Reg Anesth Pain Med. 2016;41(2):221-8.

36. Kalvoy $H$, Sauter AR. Detection of intraneural needle-placement with multiple frequency bioimpedance monitoring: a novel method. J Clin Monit Comput. 2016;30(2):185-92.

37. Chandra A, Eisma R, Felts P, Munirama S, Corner GA, Demore CE, et al. The feasibility of microultrasound as a tool to image peripheral nerves. Anaesthesia. 2017;72(2):190-6.

38. Schols RM, ter Laan M, Stassen LP, Bouvy ND, Amelink A, Wieringa FP, et al. Differentiation between nerve and adipose tissue using wide-band (350-1,830 nm) in vivo diffuse reflectance spectroscopy. Lasers Surg Med. 2014;46(7):538-45.

39. Gibbs-Strauss SL, Nasr KA, Fish KM, Khullar O, Ashitate Y, Siclovan TM, et al. Nerve-highlighting fluorescent contrast agents for image-guided surgery. Mol Imaging. 2011;10(2):91-101.

40. Whitney MA, Crisp JL, Nguyen LT, Friedman B, Gross LA, Steinbach P, et al. Fluorescent peptides highlight peripheral nerves during surgery in mice. Nat Biotechnol. 2011;29(4):352-6.

41. Gustafson TP, Yan Y, Newton P, Hunter DA, Achilefu S, Akers WJ, et al. A NIR Dye for Development of Peripheral Nerve Targeted Probes. Medchemcomm. 2012;3(6):685-90. 



\section{Chapter}

\section{Valorization}

Valorization: "Het proces van waarde-creatie uit kennis, door kennis geschikt en/of beschikbaar te maken voor maatschappelijke (en/ of economische) benutting en geschikt te maken voor vertaling in concurrerende pro-ducten, diensten, processen en nieuwe bedrijvigheid" aangepaste definitie op basis van Landelijke Commissie Valorisatie 2011 



\subsection{Valorization}

Valorization: based on pragmatic analysis of the relevance, risks, and benefits of the use of spectral tissue sensing in percutaneous needle-based procedures in the anesthesiology.

\subsection{Relevance}

In health care, patient safety, quality of life, and costs are important issues. Therefore, it is no surprise that much research is focused on these items.

In this thesis, we analyzed and evaluated the value of spectral tissue sensing in percutaneous needle-based procedures.

Safety aspects of interventional needle-based procedures in regional anesthesia and pain medicine were discussed and placed into historical perspective. The shortcomings, sensitivity, and specificity of the currently used techniques were described.

As already said, despite the use of ultrasound, radiography, nerve stimulation, and other precautions during injections, cases of nerve damage are still reported. ${ }^{1-3}$ Patients and clinicians fear neurological complications of nerve blocks, such as muscle weakness, neuropathic pain, or seizures, due to accidental intravascular injection of local anesthetics or nerve damage. ${ }^{4,5}$ Even transient neurological complications may have an impact on the quality of life of the involved patients. More serious neurological complications may occur after interventional pain therapy at the spinal level. Reports of spinal cord damage, brain infarctions, and death have been published. ${ }^{5-11}$

In this thesis, the hypothesized value of STS during interventional locoregional and interventional pain procedures was evaluated. It was supposed that the information provided by STS about the tissue in front of the needle tip could improve the correct needle tip position and improve the performance and safety of interventional needlebased procedures performed in regional anesthesia and pain therapy.

The optical characteristics of different tissues were studied, and with these results, the STS system was developed. The information obtained at the needle tip was compared with the standard or good clinical practice at this moment (aspiration, ultrasound-guided, live fluoroscopy, and DSA). We developed an optical tissue database to render STS as a valuable tool for percutaneous needle-based procedures in (locoregional) anesthesia and pain practice. This thesis focused on the optical identification of adipose tissue, muscle, blood, and nerve fascicles. We proved that STS can reliably identify blood and has the potential to detect tissue transition of the needle tip during the procedure, and the optical characteristics of fascicular nerve tissue are described.

All these potentials of STS suggest a theoretical potential to lower the complication rate during these procedures. Generally, it is nearly impossible to clearly prove a significantly lower incidence of complications of a new technology in the field of locoregional 
anesthesia and pain medicine. This is due to the low reported incidence of the complications and the very high inclusion number for such a study.

We conclude that the STS system has the potential to be clinically relevant and could improve the safety of regional anesthesia and pain medicine.

\subsection{Patient and professional safety}

Complications in health care are multifactorial and, for all involved parties, upsetting. In the field of anesthesia, nerve damage is a known complication. In a closed claims analysis, nerve damage has accounted for 22\% of claims since 1990.

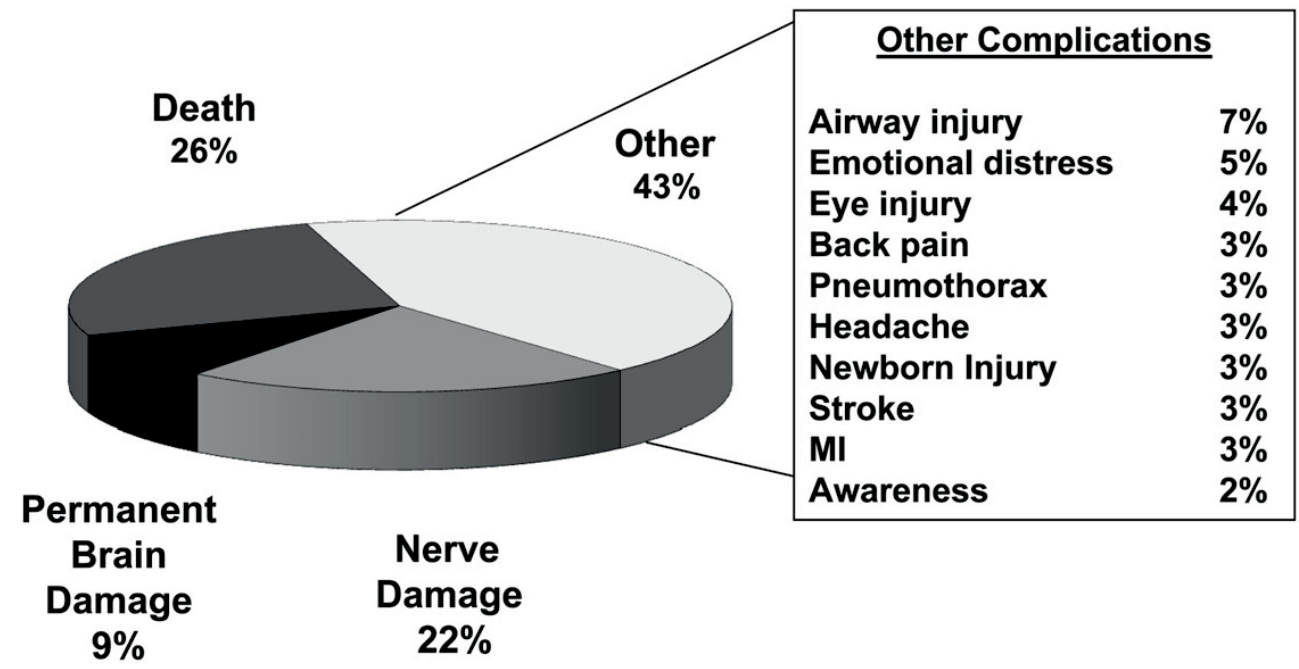

Figure 8.1: Most common complications- 1990 or later $(n=5230)$. Data from the closed claims project [12].

The most common events leading to injury in anesthesia were regional block-related; $20 \%$ of claims in 1990-2007 were related to these injuries. In perspective, claims related to the respiratory system or cardiovascular system accounted for $17 \%$ and $13 \%$, respectively. ${ }^{12}$

Market reports estimate that worldwide, 12 million nerve blocks are performed per year. The growth per year is estimated at 3-4\% for regional anesthesia and $10 \%$ for nerve blocks for chronic pain treatment.

A growing body of evidence indicates that the use of regional anesthesia offers many advantages over general anesthesia, not only in terms of reducing perioperative complications but also regarding resource utilization and patient satisfaction. ${ }^{13}$ Besides the growing popularity of regional anesthesia, the patient population is changing, and the indications for regional anesthesia may grow. 
The average age of the world's population is increasing. According to the US Census, the age group $\geq 65$-years is expected to more than double between 2014 and 2060, increasing to 98.1 million (23.6\% of the total population of the US). ${ }^{14}$ This also has an impact on the amount of surgery performed in older patients. ${ }^{15,16}$ As such, the care of older surgical patients is of increasing importance. For instance, in this population, it is of great importance to achieve adequate pain control. Untreated pain or oligoanalgesia may cause delirium and cognitive dysfunction. ${ }^{17}$ Recent evidence from the SAGES (Successful Aging after Elective Surgery) trial has demonstrated that postoperative delirium is a distinct predictor of other adverse events after surgery. A combination of delirium with other postoperative complications leads to the poorest long-term outcomes and adversely affects hospital length of stay, rates of institutionalized discharge, and 30-day readmission rates. ${ }^{18,19}$.

The American Geriatrics Society recommends the use of postoperative regional analgesia when possible to minimize the administration of opioids in order to avoid opioidrelated side effects, such as nausea, vomiting, disturbed sleep, and respiratory depression. $^{20-22}$

In experienced hands, it seems likely that peripheral nerve block would be safer than general anesthesia. However, evidence to prove this assumption will never be available due to the low numbers of severe anesthesia-related complications. ${ }^{23}$

Also, in the field of chronic pain treatment, an increase in patient numbers is anticipated. Invasive analgesic therapies provide an alternative to the medical management of chronic pain. With the increasing incidence of chronic pain worldwide, more therapies have evolved to address the growing need for pain relief options. These therapies include spinal injections, nerve blocks, radiofrequency ablation, neurostimulation, and intrathecal drug delivery. ${ }^{24}$

Altogether, the amount of needle-based percutaneous procedures is expected to grow in the next decade, and the need to improve the safety of these procedures will grow due to the more vulnerable population. There is a clear need to improve needlebased procedures and to ease the needle tip identification during the procedure to reduce the number of complications.

\subsection{Improved technical accuracy}

The STS system provides information about the final needle position and offers the possibility to document this in the patient files. This information may prove beneficial in legal procedures in case neurological damage occurs after an interventional procedure. But, the STS system has more to offer.

Table 7.1 shows the additive value of STS and the shortcomings of commonly used techniques of nerve localization. As can be seen from Table 7.1, STS scored best com- 
pared with the other tools. The combination of ultrasound and STS fulfils 12 of the 14 criteria. For instance, aspiration can be fully replaced by STS. For x-ray-based procedures, the injection of contrast fluid and DSA could be replaced by STS. The reduction of $x$-ray and contrast fluid administration is beneficial for the patient and the clinician: less exposure to $\mathrm{x}$-rays and no administration of contrast fluids. Contrast fluids have wellknown complications, like allergy and impairment of kidney function. ${ }^{25}$

More information on the tissue characteristics in front of the needle tip could also improve the training of residents. Ultrasound images and anatomical knowledge could be confirmed in real time with the use of optical tissue information.

\subsection{Summary}

STS is positioned as an innovative technology that could improve the technical accuracy and safety in a growing, more complicated, and vulnerable patient population. 


\section{References}

1. Neal, J.M., Ultrasound-guided regional anesthesia and patient safety: An evidence-based analysis. Reg Anesth Pain Med, 2010. 35(2 Suppl): p. S59-67.

2. Terkawi, A.S., et al., Ultrasound for the anesthesiologists: present and future. ScientificWorldJournal, 2013. 2013: p. 683685.

3. Neuburger, M., et al., [Localization of peripheral nerves. Success and safety with electrical nerve stimulation]. Anaesthesist, 2014. 63(5): p. 422-8.

4. Barrington, M.J. and G.L. Snyder, Neurologic complications of regional anesthesia. Curr Opin Anaesthesiol, 2011. 24(5): p. 554-60.

5. Houten, J.K. and T.J. Errico, Paraplegia after lumbosacral nerve root block: report of three cases. Spine J, 2002. 2(1): p. 70-5.

6. Malhotra, G., A. Abbasi, and M. Rhee, Complications of transforaminal cervical epidural steroid injections. Spine (Phila Pa 1976), 2009. 34(7): p. 731-9.

7. Candido, K.D. and N. Knezevic, Cervical epidural steroid injections for the treatment of cervical spinal (neck) pain. Curr Pain Headache Rep, 2013. 17(2): p. 314.

8. Helm, S., et al., A medical-legal review regarding the standard of care for epidural injections, with particular reference to a closed case. Pain Physician, 2010. 13(2): p. 145-50.

9. Murthy, N.S., T.P. Maus, and C.L. Behrns, Intraforaminal location of the great anterior radiculomedullary artery (artery of Adamkiewicz): a retrospective review. Pain Med, 2010. 11(12): p. 1756-64.

10. Huntoon, M.A. and D.P. Martin, Paralysis after transforaminal epidural injection and previous spinal surgery. Reg Anesth Pain Med, 2004. 29(5): p. 494-5.

11. Kennedy, D.J., et al., Paraplegia following image-guided transforaminal lumbar spine epidural steroid injection: two case reports. Pain Med, 2009. 10(8): p. 1389-94.

12. Metzner, J., et al., Closed claims' analysis. Best Pract Res Clin Anaesthesiol, 2011. 25(2): p. 263-76.

13. Cozowicz, C., et al., Trends in the Use of Regional Anesthesia: Neuraxial and Peripheral Nerve Blocks. Reg Anesth Pain Med, 2016. 41(1): p. 43-9.

14. Bureau, U.C. 2014 National Population Projections: Summary Tables 2014. [cited 31 July 2015.

15. Klopfenstein, C.E., et al., The influence of an aging surgical population on the anesthesia workload: a tenyear survey. Anesth Analg, 1998. 86(6): p. 1165-70.

16. Etzioni, D.A., et al., The aging population and its impact on the surgery workforce. Ann Surg, 2003. 238(2): p. 170-7.

17. Vaurio, L.E., et al., Postoperative delirium: the importance of pain and pain management. Anesth Analg, 2006. 102(4): p. 1267-73.

18. Gleason, L.J., et al., Effect of Delirium and Other Major Complications on Outcomes After Elective Surgery in Older Adults. JAMA Surg, 2015. 150(12): p. 1134-40.

19. Murthy, S., et al., Controversies in anaesthesia for noncardiac surgery in older adults. Br J Anaesth, 2015. 115 Suppl 2: p. ii15-25.

20. Rashiq, S., et al., Efficacy of supplemental peripheral nerve blockade for hip fracture surgery: multiple treatment comparison. Can J Anaesth, 2013. 60(3): p. 230-43.

21. Zhang, H., et al., Strategies for prevention of postoperative delirium: a systematic review and metaanalysis of randomized trials. Crit Care, 2013. 17(2): p. R47.

22. American Geriatrics Society Expert Panel on Postoperative Delirium in Older, A., Postoperative delirium in older adults: best practice statement from the American Geriatrics Society. J Am Coll Surg, 2015. 220(2): p. 136-48 e1.

23. Kettner, S.C., H. Willschke, and P. Marhofer, Does regional anaesthesia really improve outcome? $\mathrm{Br} J$ Anaesth, 2011. 107 Suppl 1: p. i90-5.

24. Smith, H., et al., The Role of Invasive Pain Management Modalities in the Treatment of Chronic Pain. Med Clin North Am, 2016. 100(1): p. 103-15.

25. Nouh, M.R. and M.A. El-Shazly, Radiographic and magnetic resonances contrast agents: Essentials and tips for safe practices. World J Radiol, 2017. 9(9): p. 339-349. 



\section{Chapter}

\section{Acknowledgments}

"There are two ways of spreading light: to be the candle or the mirror that reflects it"

Edith Wharton (1862-1937) American novelist, short story writer, and designer 



\subsection{Acknowledgments}

After an intensive journey, today is the day: writing this note of thanks is the finishing touch on my thesis. It has been a period of intense learning for me, not only in the scientific arena but also on a personal level.

This dedication is split into several ways:

to my promotores

to all of my co-authors

to my colleagues

all companions on my path

to my friends and family

Thank you all for your support, for the intensive and enthralling discussions, for shared laughter and tears, for the answers, for exceptional occasions to exchange inspiration and ideas, for the achievements, and for your valuable time that you spent with or gave to me. I'm very grateful for this journey.

I would like to thank all members of the scientific committee for their judgment, time, and endorsement of this thesis.

I would like to thank all of the scientific companions on my path from my time as a student to now. Each of you formed my researcher's personality in his own manner.

I must express my very profound gratitude to my family and friends for providing me with unfailing support and continuous encouragement throughout my years of study and through the process of researching and writing this thesis. This accomplishment would not have been possible without you. Thank you. 


\section{Promotieteam}

Prof. Dr. Maarten van Kleef, beste Maarten, veel dank voor de kans om deze uitdaging aan te gaan. Bedankt voor de mogelijkheid mij in het thema optische technieken te verdiepen en de toepassingsmogelijkheden binnen het veld van anesthesiologie en pijn geneeskunde te exploreren. Ik stel de open, directe en eerlijke gesprekken die wij gevoerd hebben erg op prijs.

Dr. Geert-Jan van Geffen, beste Geert-Jan, veel dank voor je ongekende enthousiasme en positiviteit. Je hebt mij door je enorme kennis en je diplomatische blik zeer geholpen.

\section{Researcher team of Philips}

Dr. Marjolein van der Voort, Dr. Torre Bydlon, Dr. Gerald Lucassen, Prof. Dr. Benno Hendriks, Dr. Geert Gijsbers, and Dr. Jeroen Kortsmit: Thank you for the scientific impact and helping me to become familiar with biomedical optics. I will never forget our sessions in the Anatomical Department at Radboud University in Nijmegen. Some cookies will always be associated with our great time.

I would also like to express my gratitude to Jan Vermeulen (Director Product Management IGT Business Incubation, Philips), Olga Crespo Biel (Marketing Manager, Business Incubation, BG Image Guided), and former managers Stefan Roggeveen and Florian Schneeberger for the great teamwork between managers and clinicians.

\section{Co-authors}

I would like to thank all of the co-authors on my papers-Dr. Adrien Desjardins, Dr. Hans van Ippel, Dr. Micha Sommer, Dr. Alfons Kessels, Prof. Dr. Jörgen Bruhn, Drs. Ivar Bruaset, and Dr. Carsten Arnoldussen and all other authors-for your encouragement, support, and guidance on the path from an idea to a study and through the word-jungle, leading from a thought over clear language into a scientific paper. Thank you for the great team performance.

\section{Afdeling Anatomie Universiteit Maastricht}

Dank gaat ook uit naar de afdeling Anatomie van de Universiteit Maastricht. Bedankt voor jullie inzet en enorme ondersteuning.

Msc. Arno Lataster, bedankt voor je pragmatische, laagdrempelige overlegmomenten en de anatomische inzichten.

Msc. Greet Mommen, bedankt voor je snelle, secure en kunstzinnige ondersteuning. 
Paul van Dijk bedankt voor je inbreng bij het uitdagende stuk over de histologie en je creativiteit om goede samples voor de NMR-analyse te verkrijgen.

\section{Studieondersteuners}

Dr. Rachel Slangen, veel dank voor je ondersteuning bij het opzetten en indienen van de METC-protocollen. Zonder jouw hulp was de weg veel langer en moeizamer geweest.

Dr. Sander van Kuijk, bedankt voor je inzichten in de statistiek. Jij hebt een groot talent om statistiek inzichtelijker te maken.

Radiologie laboranten, vooral Miriam Huskens, bedankt voor jullie onbureaucratische hulp bij de doorvoering van de doorlichting en de DSA-metingen. Er was altijd de juiste C-boog op de pijn-interventie kamer.

\section{Paranimfen}

Dr. Ankie Hamaekers en Dr. Gerald Lucassen, jullie hebben beide een belangrijke rol voor mij tijdens deze reis ingenomen. Met name jullie kritische en prikkelende vragen, juiste woorden, steun om dingen te prioriteren en jullie bijstand in de afrondende fase. Oneindig dank hiervoor.

\section{Directe Collegae uit het MUMC+}

Als medisch specialist is het niet mogelijk om zonder collegae te promoveren. Zij hebben het mogelijk gemaakt dat ik tussen tijd kreeg om aan deze thesis te kunnen werken. Zij hebben mij ondersteund met goede gedachten en tips.

In het bijzonder dank aan:

Dr. med. Markus Janssen, je hebt altijd een luisterend oor voor mij en je zeer kritische kijk op de dingen heeft mij vaak erg geholpen.

Drs. Carine Vossen, je hebt mij met zeer wijze woorden in goede en moeilijke tijden bij gestaan en nieuwe deuren voor mij geopend.

Drs. Nicole Engel, veel dank voor je ondersteuning om gedachte in woorden te vormen en mijn punt te verduidelijken. Jij hebt in mijn ogen een ongelooflijk talent!

Dr. med. Stefan Wiese, veel dank voor de brainstormsessies en goede tips tijdens onze carpool uren.

Prof. Dr. Wolfgang Buhre, bedankt voor je wetenschappelijke ondersteuning bij het schrijven van een artikel en het vertrouwen in mij om toekomstige wetenschappelijke projecten op te pakken. 
Prof. Dr. Bert Joosten, bedankt voor je begeleiding op helikopter view van mijn promotie en voor de toekomstige wetenschappelijke mogelijkheden die jij mij biedt.

Drs. Bas Timmerman, bedankt voor je geduld en inspanningen om voor mij onderzoekstijd in te plannen.

\section{People who formed my personality as a researcher}

Prof. Dr. Marcel Durieux (Anesthesiologist, University of Virginia Health System, Charlottesville, Virginia; and University of Vermont College of Medicine, Burlington, Vermont, United States of America) het is lang geleden, maar in de korte tijd waar je in Maastricht was mocht ik tijdens mijn studie Geneeskunde een kijk in je onderzoekslaboratorium nemen. De manier hoe je mensen waardeerde en aanstuurde is nog steeds een voorbeeld voor mij.

Prof. Dr. Emile Beuls (Neurologisch Expertisebureau, Universiteit Gent, België), Drs. Erwin Cornips (Neurochirurg, Maastricht Universitair Medisch Centrum, Nederland) en Prof. Dr. Johan Vles (Maastricht Universitair Medisch Centrum, Nederland). Tijdens mijn coschap neurologie heb ik de kans gekregen om samen met jullie mijn eerste internationale publicatie te schrijven. De zeer fijne manier van samenwerken heeft nog steeds een voorbeeldfunctie voor mij.

Prof. Dr. Marco Marcus (Anesthesiologist, Hamad Medical -Corporation, Doha, Qatar), tijdens mijn wetenschapsstage gedurende mijn studie Geneeskunde en mijn opleiding tot anesthesiologie heb je mijn wetenschappelijke vorming voortgezet. Veel dank hiervoor.

Prof. Dr. Robert Jan Stolker (Anesthesioloog, Erasmus Medisch, Centrum, Rotterdam, Nederland) en Prof. Dr. André van Zundert (Anesthesiologist, The University of Queensland \& Royal Brisbane \& Women's Hospital, Brisbane, Queensland, Australia) in mijn tweede jaar van mijn opleiding tot Anesthesioloog heb ik in het Catherina Ziekenhuis te Eindhoven van jullie geleerd hoe het mogelijk is ook onder drukke werkomstandigheden wetenschappelijk onderzoek te kunnen doen.

PD Dr. Susanne Weis (Scientific Researcher, System Neuroscience, Heinrich-HeineUniversität Düsseldorf, Germany) und Prof. em. Dr. Walter Huber (Sektion Kognitive Neurologie an der Neurologischen Klinik Universitätsklinikum der RWTH Aachen University), vielen Dank für die wissenschaftliche Formung während meines Dr. med. und die methodologische Bildung in bildgebenden Verfahren. Danke für mein erstes Poster auf einem internationalen Kongress.

Dr. Jaap Patijn (Neuroloog, Maastricht Universitair Medisch Centrum, Nederland), hartelijk dank voor de diepgaande discussies, de wijze worden en de wetenschappelijke inzichten. Jij bent voor mij een arts zonder grenzen op de positiefste manier. 
Prof. Dr. Angela Maas (Cardioloog, Radboud Universitair Medisch Centrum, Nijmegen, Nederland), hartelijk dank voor de wijze worden en goede adviezen. Jij hebt mij geholpen mijn wetenschappelijke kompasrichting te bepalen. Ik heb nog vaak aan jouw tips teruggedacht.

Dr. Marcus Lancé (Anesthesiologist, Hamad Medical Corporation, Doha, Qatar), bedankt voor je goede en praktische tips om bijvoorbeeld de originele tekst van historische literatuur op te vragen.

Drs Mark Siemonsma (Anesthesioloog, Ziekenhuis Gelderse Vallei, Ede, Nederland) en Drs. Jelmer Bergsma (Anesthesioloog, IJsselland Ziekenhuis, Rotterdam, Nederland), samen als vrienden en redacteurs hebben wij een mooi boek geschreven. Ik heb van jullie taalkundigheid en diplomatiek geleerd en denk graag aan onze gespreken en redactiebijeenkomsten terug.

Dr. Richel Lousberg (Onderzoeker, Afdeling Psychiatrie en Psychologie, Universiteit Maastricht, Nederland) veel dank voor je tijd om samen met Carine Vossen over nieuwe research ideeën te brainstormen en dat ik gebruik mocht maken van jouw enorme kennis om vragenlijsten te analyseren. Je menselijkheid en teamspirit hebben een voorbeeldfunctie voor mij.

\section{Maastricht Universitair Medisch Centrum}

Secretariaat, Poli Anesthesiologie en Chirurgisch Dagcentrum, hartelijk dank voor jullie inzet en tijd die jullie mij, met name organisatorisch, ondersteund hebben.

Aan de Universiteit Maastricht en het Universitair Ziekenhuis Maastricht heb ik mijn opleiding als arts, anesthesioloog en wetenschapper te danken. Door de mogelijkheden die mij geboden werden kon ik mijn weg snel en duidelijk vinden.

Ook grote dank aan alle patiënten en vrijwilligers die bereid waren aan mijn onderzoeken mee te doen.

\section{Vrienden en Freunde}

Beste vrienden, bedankt voor de tijd om elkaar niet te zien, jullie geduld als afspraken niet door gingen, de tijd voor afleiding en de uren die wij van gedachten konden wisselen.

Liebe Freunde, vielen Dank, dass Ihr mich nicht vergessen habt in der Zeit, in der ich mich nicht bei Euch gemeldet habt. Danke für Eure unterstützenden und aufbauenden Worte. Danke für Euer Zuhören. 
Liebe Lea Döhmen und Madé Rieckerhof, danke für Euere Zeit, die Ihr so spontan mit Jarno verbracht habt. Hierdurch konnte ich mit besserem Gewissen meine Zeit hinter dem Rechner verbringen.

Liebe Aikidokas, ich bedanke mich bei Euch für die Zeit, die Ihr mich auf andere Gedanken gebracht habt und mich auf den Boden der Tatsachen geholt habt, mit welchem Hebel auch immer. Dōmo arigatō gozaimashita.

\section{Familie}

Léive Paul, ech denken gären un eis Zäit an den 80er an eisem Laboratoire zu Bartreng. Zu dëser Zäit hunn ech meng Virwëtz a Forschungsgeescht entdeckt. Villmols Merci.

Liebe Rosi, danke für Deinen bedingungslosen Glauben an mich und die freie und aufgeschlossene Erziehung.

Lieber Benedikt und Raphaël, als Brüder habt Ihr mir immer ein gutes Familiengefühl gegeben und mir auch meine Möglichkeiten und Grenzen gezeigt. Danke hierfür.

Liebe Inge, danke für die tollen Gespräche und konstruktiven Vorschläge knifflige Situationen zu meistern.

Lieber Ralf, danke für Deine bedingungslose Unterstützung. Danke, dass Du Deine Bedürfnisse zurückgestellt hast und danke für die Diskussion über die diversen physikalischen Themen und Dein scharfes Auge.

Lieber Jarno, Du hast mein Leben unheimlich bereichert. Ich danke Dir für die Zeit, die ich von Dir bekommen hab und für Deine unbesiegbare und ansteckende gute Laune. 




\section{Chapter}

\section{Curriculum vitae and list of publications}

"Ever try. Ever fail. No matter Try again. Fail again. Fail better"

Samuel Beckett (1906-1989) Irish writer 



\subsection{Curriculum vitae}

Andrea Balthasar was born on June 26, 1979 in Filderstadt (Germany). She is the oldest child of a German-Luxembourgish couple. After graduating from secondary school in 1998 in Trier (Germany), she was accepted for medical studies in Maastricht. In her first year, she learned Dutch at the Language School of University of Maastricht. Her medical training started in 1999 at the University of Maastricht. During her studies, she spent her extracurricular time, her "keuzecoschap" and "wetenschapsstage," in the Department of Anesthesiology of Maastricht University Medical Center (MUMC+), The Netherlands.

In 2006, she started her postdoctoral training in anesthesiology at MUMC+ and Catharina Hospital Eindhoven, The Netherlands. In the final year of her training, she followed a tailored internship of 6 months in the chronic pain department. In 2011, she was recognized by the Dutch Board of Anesthesiologists, was additionally registered as a pain specialist, and started a 9-month fellowship in chronic pain (Prof. Dr. Maarten van Kleef) in MUMC+.

In June 2011, she passed successfully the F.I.P.P. exam, Fellow of Interventional Pain Practice, of the World Institute of Pain. Since September 2011, she has been a staff member in the Department of Anesthesiology and Pain Medicine at MUMC+.

In 2013, she passed the colloquium Dr. med. at RWTH Aachen, Germany: A supramodal brain substrate of phonological word form processing - an fMRI study on homonym finding with auditory and visual input, cum laude. The promotor team consisted of Prof. em. Dr. Walter Huber and PD Dr. Susanne Weis.

In the past several years, in addition to her clinical work, she has functioned as a faculty member of WIP-BeNeLux, provided several ultrasound courses, undertaken leadership training (Avicenna), Academy for Leadership, Leuven, Belgium, and participated as a co-editor and author of the book Probleem georiënteerd denken in de pijngeneeskunde (Tijdstroom 2017).

Her present areas of interest are neuroanesthesia, locoregional anesthesia, and teaching. Current research projects are being performed with Drs. Carine Vossen, Richel Lousberg, and Pieter Emans on postoperative pain measurements, using digital Experience Sampling Method, the PsyMate app. In the near future, she will additionally concentrate on the role of genes in perioperative pain development.

Andrea is married to Ralf Balthasar-Dewender, and they have a son, Jarno. Andrea loves to travel and has been training in Aikido for many years. 


\subsection{Publications}

\subsubsection{International publications}

1. Balthasar et al. Optical signature of nerve tissue - Explorative ex vivo study comparing optical, histological, and molecular characteristics of different adipose and nerve tissues. Under review January 2018 Lasers in Surgery and Medicine.

2. Beugels, Hommes, Balthasar, et al. Autologous fat transfer as a treatment for nonneuroma peripheral neuropathic pain. Submitted January 2018 Plastic and Reconstructive Surgery.

3. Langhout, Bydlon, Balthasar et al. Nerve detection using optical spectroscopy, an evaluation in four different models: in human and swine, in vivo, and post mortem. Lasers Surg Med. 2017 Nov 21. doi: 10.1002/lsm.22755.

4. Balthasar et al. Spectral tissue sensing to identify intra-and extravascular needle placement- A randomized single-blind controlled trial. PLoS ONE 2017 12(3): e0172662. https://doi.org/10.1371/journal.pone.0172662.

5. Bouman, Sieben, Balthasar et al. Boundaries of the thoracic paravertebral space: potential risks and benefits of the thoracic paravertebral block from an anatomical perspective. Surg Radiol Anat. 2017 Apr 25. doi: 10.1007/s00276-017-1857-4.

6. Balthasar et al. The role of spectral tissue sensing during lumbar transforaminal epidural injection. Reg Anesth Pain Med. 2016 Jul-Aug;41(4):520-6.

7. Hendriks, Balthasar et al. Nerve detection using diffuse optical spectroscopy from 400 to $1710 \mathrm{~nm}$ : a comparison of classification methods. J Transl Med (2015) 13:380.

8. Balthasar et al. Optical Detection of Peripheral Nerves: An in vivo Human Study. Reg Anesth Pain Med. 2012 May-Jun;37(3):277-82.

9. Balthasar et al. Optical Detection of Vascular Penetration during Nerve Blocks: An in vivo Human Study. Reg Anesth Pain Med. 2012 Jan-Feb;37(1):3-7.

10. Balthasar et al. A supramodal brain substrate of phonological word form processing -an fMRI study on homonym finding with auditory and visual input. Brain Research. 2011 Sep 2.

11. Balthasar et al. Analysis of the success and failure of endoscopic third ventriculostomy in infants less than 1 year of age. Childs Nerv Syst. 2006 Sep 9.

\subsubsection{National publications}

1. Van Riel, Balthasar, et al. Stress-induced cardiomyopathy due to accidental injection of norepinephrine instead of neostigmine. Under review January 2018 Nederlands Journal of Critical Care. 
2. Balthasar et al. Eagle Syndroom: a case report. Nederlandse Tijdschrift voor Pijn en Pijnbestrijding, 2011, Nr 45.

3. Balthasar et al. Opiaten op recept. Een probleem in Nederland? Nederlandse Tijdschrift voor Pijn en Pijnbestrijding, 2010 Nr 44.

4. Patijn, Lataster, Balthasar et al. Megadolichobasilaris en Trigeminus Neuralgie: een case-report. Nederlandse Tijdschrift voor Pijn en Pijnbestrijding, 2010 Nr 44.

5. Balthasar et al. Acute postoperatieve pijn. Nederlands tijdschrift voor Anesthesiologie 2009 Apr 21.

6. van Gorp, Krommendijk, Marcus, Balthasar et al. Do we need a national guideline for obstetric analgesia in the Netherlands? Evaluation of a questionnaire among Dutch anesthetists. Nederlands tijdschrift voor Anesthesiologie 2005 Dec 18-4.

\subsubsection{Book Editor}

- Editor and coauthor of the book (Problem based thinking in painmedicine): Probleemgeoriënteerd denken in de pijngeneeskunde. Een praktijkboek voor de opleiding en de kliniek. Balthasar, Bergsma, Siemonsma, De Tijdstroom, 2017.

\subsubsection{Book Chapters:}

1. Een man met pijn in zijn been; Balthasar, de Wolf, Probleemgeoriënteerd denken in de pijngeneeskunde, De Tijdstroom, 2017,

2. Hetepeperpleister; Vanhentenrijk, Balthasar, Probleemgeoriënteerd denken in de pijngeneeskunde, De Tijdstroom, 2017.

3. Een ronde buik en een brandend bovenbeen; Bral, Balthasar, Probleemgeoriënteerd denken in de pijngeneeskunde, De Tijdstroom, 2017

4. Een consult op de afdeling oncologie; Janssen, Balthasar, Siemonsma, Probleemgeoriënteerd denken in de pijngeneeskunde, De Tijdstroom, 2017

5. Ik mis mijn onderbeen! de Waal, Balthasar, Snijdelaar, Probleemgeoriënteerd denken in de pijngeneeskunde, De Tijdstroom, 2017

6. Pijnlijke pink na vuurwerktrauma; Oomen, Balthasar, Probleemgeoriënteerd denken in de pijngeneeskunde, De Tijdstroom, 2017

7. Pijn in de laatste levensfase; Bral, Balthasar, Bergsma, Probleemgeoriënteerd denken in de pijngeneeskunde, De Tijdstroom, 2017

\subsection{Posters and presentations}

2017 Poster presentation ESRA congress, Lugano, $\mathrm{CH}$; Is Ultrasound, with or without Additional Instruments or Techniques, the Ideal Guidance Tool in 
Locoregional Anesthesia? Results from an International Survey of 14 Criteria. Segers, Theunissen, van Geffen, Balthasar.

2013 -2017 Speaker; Course: Oncologische Pijn en Palliatieve Zorg: De WHO pijnladder.

2015 Presentation: "Naald met ogen" Weefselherkenning door middel van licht, DARA Symposium, Heeze, The Netherlands.

2014 Presentation: Spectral tissue sensing - eyes to the needle. $7^{\text {th }}$ World Congress World Institute of Pain, Maastricht, Mai 2014.

2013 Poster presentation ESRA congress, Glasgow, UK; Optical sensing: a new technique for providing tissue information at the needle tip. Balthasar et al.

2012 Poster presentation ESRA congress, Bordeaux, France; Optical Detection of Peripheral Nerves: An in vivo Human Study. Balthasar et al.

2012 Poster presentation WIP congress Miami Beach, USA; Optical Detection of Vascular Penetration during Nerve Blocks: An in vivo Human Study. Balthasar et al.

2011 Poster presentation NVIC dagen, Ede; Stress-induced transient cardiomyopathy due to accidental administration of norepinephrine instead of neostigmine. Balthasar et al.

2009 Poster presentation NVA dagen, Maastricht; Postoperative care for elective neurosurgical patients: Is PACU/NMC recovery a safe alternative for postoperative ICU monitoring? Vossen, Theunissen, Balthasar et al.

2009 Poster presentation 27th European Workshop on Cognitive Neuropsychology Bressanone, Italie; A supramodal brain substrate of abstract word form processing -an fMRI study with visual and auditory input to homonym finding. Balthasar et al.

2006 Poster presentation NVA dagen, Maastricht; Chronic pain after day-case surgery: is there an association with acute pain? Balthasar et al. 
